\title{
The Temporal Ultraviolet Limit
}

\author{
Tadeusz Balaban \\ Department of Mathematics, Rutgers University, 110 Frelinghuysen Rd, Piscataway, NJ \\ 08854-8019, USA \\ Joel Feldman \\ Department of Mathematics, University of British Columbia, Vancouver, BC, Canada V6T1Z2 \\ Horst Knörrer and Eugene Trubowitz \\ Mathematik, ETH, 8092 Zürich, Switzerland
}





\section{Contents}

1 The Temporal Ultraviolet Limit 1

1.1 Introduction 1

1.2 Motivation for the Stationary Phase Approximation 12

1.3 Bounds on the Stationary Phase Approximation 19

1.4 Functional Integrals 28

1.5 A Simple High Temperature Expansion 42

Appendix A Complex Gaussian Integrals $\quad 59$

$\begin{array}{ll}\text { References } & 64\end{array}$ 


\section{1}

\section{The Temporal Ultraviolet Limit}

\subsection{Introduction}

\subsubsection{The Physical Setting}

These lectures ${ }^{1}$ concern the first, relatively small, step in a program whose long-term goal is the, mathematically rigorous, construction of a standard model of a gas of bosons. Even this first step is too long and complicated to present completely here. But I will outline it and highlight a couple of the tools employed that tend to crop up quite commonly in constructions of quantum field theories and many-body models. The model of our gas of bosons is based on the following assumptions.

- Each particle in the gas has a kinetic energy. The corresponding quantum mechanical observable is an operator $h$. The most commonly used $h$ is $-\frac{1}{2 m} \Delta$, which corresponds to the classical kinetic energy $\frac{\mathbf{p}^{2}}{2 m}$. (Balaban et al., 2010c) allows a more general class of operators like this.

- The particles in the gas interact with each other through a translationally invariant, exponentially decaying, strictly positive definite two-body potential, $2 v(\mathbf{x}, \mathbf{y})$.

- The system is in the thermodynamic equilibrium given by the grand canonical ensemble with temperature $T>0$ and chemical potential $\mu \in \mathbb{R}$. We shall not place any further restrictions on $T$ and $\mu$. But the most interesting temperatures are small and the most interesting chemical potentials are small and positive.

\subsubsection{The Physics of Interest}

I'll formulate the model mathematically, carefully, later. But to get a first hint both of the expected physical behaviour and of the formalism that we shall use, consider the following, formal, functional integral representation of the partition function for this system. This representation is commonly used in the Physics literature. See, for example, (Negele and Orland, 1988, (2.66)).

$$
\operatorname{Tr} e^{-\frac{1}{k T}(H-\mu N)}=\int \prod_{\substack{\mathbf{x} \in \mathbb{R}^{3} \\ 0<\tau \leq \frac{1}{k T}}} \frac{d \alpha_{\tau}(\mathbf{x})^{*} \wedge d \alpha_{\tau}(\mathbf{x})}{2 \pi i} e^{\mathcal{A}\left(\alpha^{*}, \alpha\right)}
$$

where $H$ is the Hamiltonian, $N$ is the number operator and the "action"

$$
\mathcal{A}\left(\alpha^{*}, \alpha\right)=\int_{0}^{\frac{1}{k T}} d \tau \int_{\mathbb{R}^{3}} d^{3} \mathbf{x} \quad \alpha_{\tau}(\mathbf{x})^{*} \frac{\partial}{\partial \tau} \alpha_{\tau}(\mathbf{x})-\int_{0}^{\frac{1}{k T}} d \tau K\left(\alpha_{\tau}^{*}, \alpha_{\tau}\right)
$$

\footnotetext{
${ }^{1}$ These notes expand upon lectures given by Joel Feldman.
} 


\section{The Temporal Ultraviolet Limit}

with

$$
\begin{aligned}
K\left(\alpha^{*}, \alpha\right)= & \iint d \mathbf{x} d \mathbf{y} \alpha(\mathbf{x})^{*} h(\mathbf{x}, \mathbf{y}) \alpha(\mathbf{y})-\mu \int d \mathbf{x} \alpha(\mathbf{x})^{*} \alpha(\mathbf{x}) \\
& +\iint d \mathbf{x} d \mathbf{y} \alpha(\mathbf{x})^{*} \alpha(\mathbf{x}) v(\mathbf{x}, \mathbf{y}) \alpha(\mathbf{y})^{*} \alpha(\mathbf{y})
\end{aligned}
$$

and $h(\mathbf{x}, \mathbf{y})$ being the kernel of the operator $h$. In the integral on the right hand side of (1.1), there is a two parameter family of integration variables. The first parameter, $\tau$, runs over the "time" interval $\left(0, \frac{1}{k T}\right]$ (the reason for the half open, half closed time interval is that there is a periodicity condition $\left.\alpha_{0}(\mathbf{x})=\alpha_{\frac{1}{k T}}(\mathbf{x})\right)$ and the second parameter, $\mathbf{x}$, runs over "space", $\mathbb{R}^{d}$. For each $\tau$ and $\mathbf{x}$, there is an integration variable, $\alpha_{\tau}(\mathbf{x})$, that runs over the complex plane, $\mathbb{C}$. For a complex variable $z=x+i y, \frac{d z \wedge d z^{*}}{2 \pi i}$ is the usual Euclidean measure $\frac{1}{\pi} d x d y$.

Thus the "measure" for the integral on the right hand side of (1.1) is a Lebesgue measure in uncountably many variables. It clearly has no mathematical meaning. But it is still a useful source of intuition. If $\alpha_{\tau}(\mathbf{x})=\Phi \in \mathbb{C}$ is a constant, independent of $\tau$ and $\mathbf{x}$, the action $\mathcal{A}\left(\alpha^{*}, \alpha\right)$ simplifies to minus the integral over $\tau$ and $\mathbf{x}$ of the "naive effective potential" $\hat{v}(0)|\Phi|^{4}-\mu|\Phi|^{2}$ where $\hat{v}(0)=\int d \mathbf{y} v(\mathbf{x}, \mathbf{y})$ (recall that $v(\mathbf{x}, \mathbf{y})$ is

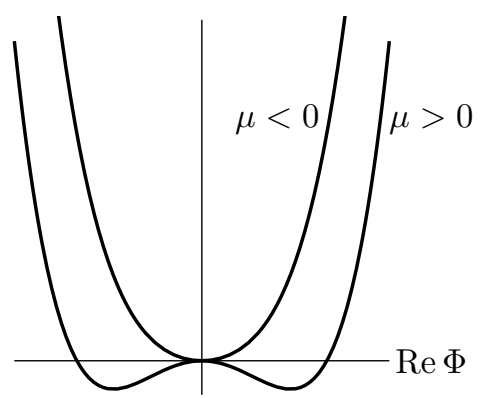

Fig. 1.1 Graph of the effective potential

translation invariant) and we have assumed and that $h$ annihilates constants and that $\hat{v}(0)>0$. This effective potential is graphed above. Its minimum is

- nondegenerate at the point $\Phi=0$ when $\mu<0$ and

- degenerate along the circle $|\Phi|=\sqrt{\frac{\mu}{2 \hat{v}(0)}}$ when $\mu>0$.

This suggests that, if the temperature is low so that fluctuations about the minimum are small, each integration variable $\alpha_{\tau}(\mathbf{x})$ tends to be localized about 0 when $\mu<0$ and tends to be localized about $\left|\alpha_{\tau}(\mathbf{x})\right|=\sqrt{\frac{\mu}{2 \hat{v}(0)}}$ when $\mu>0$. To help us glean some more detailed intuition from the formal functional integral, we introduce "Euclidean time evolving" annihilation and creation operators

$$
a(\tau, \mathbf{x})=e^{(H-\mu N) \tau} a(\mathbf{x}) e^{-(H-\mu N) \tau} a(\mathbf{x}) \quad a^{\dagger}(\tau, \mathbf{x})=e^{(H-\mu N) \tau} a^{\dagger}(\mathbf{x}) e^{-(H-\mu N) \tau} a^{\dagger}(\mathbf{x})
$$

and notation for "expectation values" both in the physical Hilbert space and with respect to the functional integral 


$$
\begin{aligned}
\left\langle f\left(a^{\dagger}, a\right)\right\rangle & =\frac{\operatorname{Tr}\left(e^{-\frac{1}{k T}(H-\mu N)} f\left(a^{\dagger}, a\right)\right)}{\operatorname{Tr} e^{-\frac{1}{k T}(H-\mu N)}} \\
\left\langle\left\langle f\left(\alpha^{*}, \alpha\right)\right\rangle\right\rangle & =\frac{\int \prod_{\mathbf{x}, \tau} \frac{d \alpha_{\tau}(\mathbf{x})^{*} \wedge d \alpha_{\tau}(\mathbf{x})}{2 \pi i} e^{\mathcal{A}\left(\alpha^{*}, \alpha\right)} f\left(\alpha^{*}, \alpha\right)}{\int \prod_{\mathbf{x}, \tau} \frac{d \alpha_{\tau}(\mathbf{x})^{*} \wedge d \alpha_{\tau}(\mathbf{x})}{2 \pi i}} e^{\mathcal{A}\left(\alpha^{*}, \alpha\right)}
\end{aligned}
$$

We will use two more functional integral representations similar to the representation (1.1) for the partition function. They are for the one and two point correlation functions

$$
\begin{aligned}
\left\langle a^{(\dagger)}(\tau, \mathbf{x})\right\rangle & =\left\langle\left\langle\alpha_{\tau}(\mathbf{x})^{(*)}\right\rangle\right\rangle \\
\left\langle a^{\dagger}(\tau, \mathbf{x}) a\left(\tau^{\prime}, \mathbf{x}^{\prime}\right)\right\rangle & =\left\langle\left\langle\alpha_{\tau}(\mathbf{x})^{*} \alpha_{\tau^{\prime}}\left(\mathbf{x}^{\prime}\right)\right\rangle\right\rangle
\end{aligned}
$$

The first is valid for $\frac{1}{k T} \geq \tau \geq 0$ and the second is valid for $\frac{1}{k T} \geq \tau>\tau^{\prime} \geq 0$. Actually, (1.4) is two formulae at once — one when the bracketed exponents are included and one when the bracketed exponents are omitted. Let us try to compute these expectation values, at least approximately.

(1) The one point function for $\mu<0$ : First consider $\mu<0$. The one point function (1.4) is zero by symmetry considerations. This can be seen by using either side of (1.4). On the right hand side, make the change of variables which rotates each integration variable by a fixed angle $\theta$. That is

$$
\alpha_{\tau}(\mathbf{x}) \rightarrow e^{i \theta} \alpha_{\tau}(\mathbf{x}) \quad \alpha_{\tau}(\mathbf{x})^{*} \rightarrow e^{-i \theta} \alpha_{\tau}(\mathbf{x})^{*}
$$

As both the measure $\frac{d \alpha_{\tau}(\mathbf{x})^{*} \wedge d \alpha_{\tau}(\mathbf{x})}{2 \pi i}$ and the action $\mathcal{A}\left(\alpha^{*}, \alpha\right)$ are invariant under this change of variables, we have

$$
\left\langle\left\langle\alpha_{\tau}(\mathbf{x})^{(*)}\right\rangle\right\rangle=e^{(-) i \theta}\left\langle\left\langle\alpha_{\tau}(\mathbf{x})^{(*)}\right\rangle\right\rangle \quad \Longrightarrow \quad\left\langle\left\langle\alpha_{\tau}(\mathbf{x})^{(*)}\right\rangle\right\rangle=0
$$

For the corresponding argument on the left hand side, we unitarily transform the Hilbert space using the operator $e^{i N \theta}$. By cyclicity of the trace

$$
\begin{aligned}
\operatorname{Tr}\left(e^{-\frac{1}{k T}(H-\mu N)} a^{(\dagger)}(\tau, \mathbf{x})\right) & =\operatorname{Tr}\left(e^{-i N \theta} e^{-\frac{1}{k T}(H-\mu N)} a^{(\dagger)}(\tau, \mathbf{x}) e^{i N \theta}\right) \\
& =\operatorname{Tr}\left(e^{-\frac{1}{k T}(H-\mu N)} e^{-i N \theta} a^{(\dagger)}(\tau, \mathbf{x}) e^{i N \theta}\right) \\
& =e^{(-) i \theta} \operatorname{Tr}\left(e^{-\frac{1}{k T}(H-\mu N)} a^{(\dagger)}(\tau, \mathbf{x})\right)
\end{aligned}
$$

The critical step was the second equality, where we used that $H-\mu N$ commutes with the number operator $N$. That is, the Hamiltonian conserves particle number. For the third equality, we used that

$$
e^{-i N \theta} a^{(\dagger)}(\tau, \mathbf{x}) e^{i N \theta}=e^{(-) i \theta} a^{(\dagger)}(\tau, \mathbf{x})
$$

Once again, we have

$$
\left\langle a^{(\dagger)}(\tau, \mathbf{x})\right\rangle=e^{(-) i \theta}\left\langle a^{(\dagger)}(\tau, \mathbf{x})\right\rangle \quad \Longrightarrow \quad\left\langle a^{(\dagger)}(\tau, \mathbf{x})\right\rangle=0
$$

It would appear that this argument also implies $\left\langle a^{(\dagger)}(\tau, \mathbf{x})\right\rangle=0$ when $\mu>0$. But there is a subtlety when $\mu>0$ that we will discuss shortly. 


\section{The Temporal Ultraviolet Limit}

(2) The two point function for $\mu<0$ : Now let's move on to the two point function (1.5) when $\mu<0$. We are expecting the most important contributions to the functional integral to come from $\alpha_{\tau}(\mathbf{x}) \approx 0$. So approximate the action $\mathcal{A}$ by dropping all terms of degree strictly bigger than two in the integration variables. That is, drop the quartic, $v(\mathbf{x}, \mathbf{y})$ part of (1.3). This turns the action into a quadratic function of the integration variables. Using (the natural formal analog of) part (a) of Lemma A.1 with $D=$ $-\frac{\partial}{\partial \tau}+h-\mu$, we have

$$
\left\langle\left\langle\alpha_{\tau}(\mathbf{x})^{*} \alpha_{\tau^{\prime}}\left(\mathbf{x}^{\prime}\right)\right\rangle=\left(-\frac{\partial}{\partial \tau}+h-\mu\right)^{-1}\left((\tau, \mathbf{x}),\left(\tau^{\prime}, \mathbf{x}^{\prime}\right)\right)\right.
$$

The right hand side is the kernel of the operator inverse of $-\frac{\partial}{\partial \tau}+h-\mu$. Because $h$ is translation invariant we can use the Fourier transform to compute it.

$$
\left(-\frac{\partial}{\partial \tau}+h-\mu\right)^{-1}\left((\tau, \mathbf{x}),\left(\tau^{\prime}, \mathbf{x}^{\prime}\right)\right)=k T \sum_{k_{0} \in 2 \pi k T \mathbb{Z}} \int_{\mathbb{R}^{3}} \frac{d^{3} \mathbf{k}}{(2 \pi)^{3}} e^{i k_{0}\left(\tau-\tau^{\prime}\right)-i \mathbf{k} \cdot\left(\mathbf{x}-\mathbf{x}^{\prime}\right)} \frac{1}{-i k_{0}+\hat{h}(\mathbf{k})-\mu}
$$

(If you were expecting minus this answer, it is probably because you forgot that the usual two-point function is defined to be $-\left\langle\left\langle\alpha_{\tau}(\mathbf{x})^{*} \alpha_{\tau^{\prime}}\left(\mathbf{x}^{\prime}\right)\right\rangle\right\rangle$.) The sum over $k_{0}$ can be evaluated exactly using a contour integral trick (see, for example, (Fetter and Walecka, 1971, (25.32)-(25.35))) giving

$$
\left\langle\left\langle\alpha_{\tau}(\mathbf{x})^{*} \alpha_{\tau^{\prime}}\left(\mathbf{x}^{\prime}\right)\right\rangle=\int_{\mathbb{R}^{3}} \frac{d^{3} \mathbf{k}}{(2 \pi)^{3}} e^{-i \mathbf{k} \cdot\left(\mathbf{x}-\mathbf{x}^{\prime}\right)} e^{(\hat{h}(\mathbf{k})-\mu)\left(\tau-\tau^{\prime}\right)}\left(e^{\frac{1}{k T}(\hat{h}(\mathbf{k})-\mu)}-1\right)^{-1}\right.
$$

For large $\mathbf{k}$ the integrand is bounded in absolute value by the exponential of minus a constant times $|\mathbf{k}|^{2}$, since $\tau-\tau^{\prime}<\frac{1}{k T}$. Furthermore the denominator never vanishes, because $\mu<0$. Both the last two sentences remain true even if, in $e^{(\hat{h}(\mathbf{k})-\mu)\left(\tau-\tau^{\prime}\right)}$ and $\left(e^{\frac{1}{k T}(\hat{h}(\mathbf{k})-\mu)}-1\right)^{-1}, \mathbf{k}$ is given a fixed, not too big, imaginary part. Consequently, $\left\langle\left\langle\alpha_{\tau}(\mathbf{x})^{*} \alpha_{\tau^{\prime}}\left(\mathbf{x}^{\prime}\right)\right\rangle\right\rangle$ decays exponentially to zero as $\left|\mathbf{x}-\mathbf{x}^{\prime}\right| \rightarrow \infty$.

(3) The one point function for $\mu>0$ : We have already seen that when $\mu>0$ the naive effective potential takes its minimum value on the circle $|\Phi|=\sqrt{\frac{\mu}{2 \hat{v}(0)}}$ in the complex plane. This suggests that the integration variables $\alpha_{\tau}(\mathbf{x})$ would like to stay near that circle. But nothing in the integral favours any phase of $\Phi$ over any other phase. Something very similar happens in magnetic materials. Indeed it can be useful to pretend that each $\alpha_{\tau}(\mathbf{x})$ represents the needle of a magnetic compass. As $\mu>0$ and the temperature is very low, the length of each needle is essentially fixed at $\sqrt{\frac{\mu}{2 \hat{v}(0)}}$. But its orientation, the argument of $\alpha_{\tau}(\mathbf{x})$, is free. If we now subject the system to an external magnetic field that favours one particular direction, all of $\alpha_{\tau}(\mathbf{x})$ 's will take values near a single $\Phi$ on the circle. If the temperature is low enough, this will remain the case even if the strength of the magnetic field is then reduced to zero. The same thing happens if, instead of applying a weak bulk magnetic field, we impose boundary conditions near infinity that favour one particular phase of $\Phi$. The moral is that the behaviour of the system, and in particular the one and two-point functions, can be expected to depend not only on the action, but also on the limiting process used to 
carefully define the system. This is a very common phenomenon in symmetry breaking scenarios.

So let's assume that our limiting process favours one particular $\Phi$. Make a change of variables

$$
\alpha_{\tau}(\mathbf{x})=\Phi+\beta_{\tau}(\mathbf{x}) \quad \alpha_{\tau}(\mathbf{x})^{*}=\Phi^{*}+\beta_{\tau}(\mathbf{x})^{*}
$$

We are expecting $\beta_{\tau}(\mathbf{x})$ to be small. Under this change of variables, the $K\left(\alpha^{*}, \alpha\right)$ of (1.3) becomes, supressing the $\tau$ subscripts and recalling that the kinetic energy operator $h$ annihilates constants,

$$
\begin{aligned}
K\left(\alpha^{*}, \alpha\right)= & \iint d \mathbf{x} d \mathbf{y} \beta(\mathbf{x})^{*} h(\mathbf{x}, \mathbf{y}) \beta(\mathbf{y}) \\
& +\int d \mathbf{x}\left[-\mu|\Phi|^{2}+\hat{v}(0)|\Phi|^{4}\right] \\
& +\int d \mathbf{x} \beta(\mathbf{x})^{*}\left[-\mu+2 \hat{v}(0)|\Phi|^{2}\right] \Phi+\int d \mathbf{x} \Phi^{*}\left[-\mu+2 \hat{v}(0)|\Phi|^{2}\right] \beta(\mathbf{x}) \\
& +\int d \mathbf{x} \beta(\mathbf{x})^{*}\left[-\mu+2 \hat{v}(0)|\Phi|^{2}\right] \beta(\mathbf{x})+2|\Phi|^{2} \iint d \mathbf{x} d \mathbf{y} \beta(\mathbf{x})^{*} v(\mathbf{x}, \mathbf{y}) \beta(\mathbf{y}) \\
& +\left(\Phi^{*}\right)^{2} \iint d \mathbf{x} d \mathbf{y} \beta(\mathbf{x}) v(\mathbf{x}, \mathbf{y}) \beta(\mathbf{y})+\Phi^{2} \iint d \mathbf{x} d \mathbf{y} \beta(\mathbf{x})^{*} v(\mathbf{x}, \mathbf{y}) \beta(\mathbf{y})^{*} \\
& \quad+O\left(|\beta|^{3}\right)+O\left(|\beta|^{4}\right)
\end{aligned}
$$

In computing the one and two-point functions, the constant (i.e. independent of $\beta$ ) term in the second row will appear both in the numerator and in the denominator and so will cancel out. So we may as well drop it. The two degree one terms in the third row and the first degree two term in the fourth row are zero because $\left|\Phi^{2}\right|=\frac{\mu}{2 \hat{v}(0)}$. We drop all terms of degree three and four in $\beta, \beta^{*}$, by way of approximation. So we end up with the action

$$
\tilde{\mathcal{A}}\left(\beta^{*}, \beta\right)=\int_{0}^{\frac{1}{k T}} d \tau \int d^{3} \mathbf{x} \beta_{\tau}(\mathbf{x})^{*} \frac{\partial}{\partial \tau} \beta_{\tau}(\mathbf{x})-\int_{0}^{\frac{1}{k T}} d \tau \tilde{K}\left(\beta_{\tau}^{*}, \beta_{\tau}\right)
$$

where

$$
\begin{aligned}
\tilde{K}\left(\beta^{*}, \beta\right)= & \iint d \mathbf{x} d \mathbf{y} \beta(\mathbf{x})^{*}\left[h(\mathbf{x}, \mathbf{y})+2|\Phi|^{2} v(\mathbf{x}, \mathbf{y})\right] \beta(\mathbf{y}) \\
& +\left(\Phi^{*}\right)^{2} \iint d \mathbf{x} d \mathbf{y} \beta(\mathbf{x}) v(\mathbf{x}, \mathbf{y}) \beta(\mathbf{y})+\Phi^{2} \iint d \mathbf{x} d \mathbf{y} \beta(\mathbf{x})^{*} v(\mathbf{x}, \mathbf{y}) \beta(\mathbf{y})^{*}
\end{aligned}
$$

This action is, of course, no longer invariant under $\beta \rightarrow e^{i \theta} \beta, \beta^{*} \rightarrow e^{-i \theta} \beta^{*}$. But it is still invariant under $\beta \rightarrow-\beta, \beta^{*} \rightarrow-\beta^{*}$. Hence

$$
\left\langle\left\langle\alpha_{\tau}(\mathbf{x})^{(*)}\right\rangle=\Phi^{(*)}+\left\langle\left\langle\beta_{\tau}(\mathbf{x})^{(*)}\right\rangle=\Phi^{(*)}\right.\right.
$$

This is nonzero and shows us that conservation of particle number has been broken.

(4) The two point function for $\mu>0$ : $\quad$ By making a change of variables $\alpha_{\tau}(\mathbf{x}) \rightarrow$ $\alpha_{\tau}(\mathbf{x}) e^{i \theta}$ we may always arrange that the favoured $\Phi$ has phase zero, so that it is 


\section{The Temporal Ultraviolet Limit}

positive. So for simplicity, we now set $\Phi=\sqrt{\frac{\mu}{2 \hat{v}(0)}}$, which we denote $\sqrt{n_{0}}$. To compute the two point functions, using the approximate action (1.7) we apply (the natural formal analog) of part (b) of Lemma A.1 with

$$
D=-\frac{\partial}{\partial \tau}+h+2 n_{0} v \quad V=W=n_{0} v
$$

Note that, because $v$ and $h$ are translationally invariant, $D$ and $V=W$ commute with each other and we may also compute with these operators using Fourier transforms. In particular, in momentum space, the operators $D, D^{t}=\frac{\partial}{\partial \tau}+h+2 n_{0} v$ and $V$ are multiplication by $-i k_{0}+\hat{h}(\mathbf{k})+2 n_{0} \hat{v}(\mathbf{k}), i k_{0}+\hat{h}(\mathbf{k})+2 n_{0} \hat{v}(\mathbf{k})$ and $n_{0} \hat{v}(\mathbf{k})$, respectively. Hence the kernel of $\left(D D^{t}-4 V^{2}\right)^{-1}$ is

$$
\begin{aligned}
\left(D D^{t}-4 V^{2}\right)^{-1} & \left((\tau, \mathbf{x}),\left(\tau^{\prime}, \mathbf{x}^{\prime}\right)\right) \\
= & k T \sum_{k_{0} \in 2 \pi k T \mathbb{Z}} \int_{\mathbb{R}^{3}} \frac{d^{3} \mathbf{k}}{(2 \pi)^{3}} e^{i k_{0}\left(\tau-\tau^{\prime}\right)-i \mathbf{k} \cdot\left(\mathbf{x}-\mathbf{x}^{\prime}\right)} \frac{1}{k_{0}^{2}+\left[\hat{h}(\mathbf{k})+2 n_{0} \hat{v}(\mathbf{k})\right]^{2}-4 n_{0}^{2} \hat{v}(\mathbf{k})^{2}} \\
= & k T \sum_{k_{0} \in 2 \pi k T \mathbb{Z}} \int_{\mathbb{R}^{3}} \frac{d^{3} \mathbf{k}}{(2 \pi)^{3}} e^{i k_{0}\left(\tau-\tau^{\prime}\right)-i \mathbf{k} \cdot\left(\mathbf{x}-\mathbf{x}^{\prime}\right)} \frac{1}{k_{0}^{2}+\hat{h}(\mathbf{k})\left[\hat{h}(\mathbf{k})+4 n_{0} \hat{v}(\mathbf{k})\right]}
\end{aligned}
$$

Combining (1.6), (three variants of) (A.1) and (A.3),

$$
\begin{aligned}
\left\langle\left\langle\alpha_{\tau}(\mathbf{x})^{*} \alpha_{\tau^{\prime}}\left(\mathbf{x}^{\prime}\right)\right\rangle\right\rangle & =n_{0}+\left\langle\left\langle\beta_{\tau}(\mathbf{x})^{*} \beta_{\tau^{\prime}}\left(\mathbf{x}^{\prime}\right)\right\rangle\right\rangle \\
& =n_{0}+k T \sum_{k_{0} \in 2 \pi k T \mathbb{Z}} \int_{\mathbb{R}^{3}} \frac{d^{3} \mathbf{k}}{(2 \pi)^{3}} e^{i k_{0}\left(\tau-\tau^{\prime}\right)+i \mathbf{k} \cdot\left(\mathbf{x}-\mathbf{x}^{\prime}\right)} \frac{i k_{0}+\hat{h}(\mathbf{k})+2 n_{0} \hat{v}(\mathbf{k})}{k_{0}^{2}+\hat{h}(\mathbf{k})\left[\hat{h}(\mathbf{k})+4 n_{0} \hat{v}(\mathbf{k})\right]} \\
\left.\left\langle\alpha_{\tau}(\mathbf{x}) \alpha_{\tau^{\prime}}\left(\mathbf{x}^{\prime}\right)\right\rangle\right\rangle & =n_{0}+\left\langle\left\langle\beta_{\tau}(\mathbf{x}) \beta_{\tau^{\prime}}\left(\mathbf{x}^{\prime}\right)\right\rangle\right\rangle \\
& =n_{0}-k T \sum_{k_{0} \in 2 \pi k T \mathbb{Z}} \int_{\mathbb{R}^{3}} \frac{d^{3} \mathbf{k}}{(2 \pi)^{3}} e^{i k_{0}\left(\tau-\tau^{\prime}\right)+i \mathbf{k} \cdot\left(\mathbf{x}-\mathbf{x}^{\prime}\right)} \frac{2 n_{0} \hat{v}(\mathbf{k})}{k_{0}^{2}+\hat{h}(\mathbf{k})\left[\hat{h}(\mathbf{k})+4 n_{0} \hat{v}(\mathbf{k})\right]}
\end{aligned}
$$

In contrast to the case $\mu<0$, these expectation values converge to $n_{0}$, rather than zero, as $\left|\mathbf{x}-\mathbf{x}^{\prime}\right| \rightarrow \infty$. This is called "long range order". Note also that the integrands have poles at

$$
k_{0}= \pm i E(\mathbf{k}) \quad \text { where } E(\mathbf{k})=\sqrt{\hat{h}(\mathbf{k})\left[\hat{h}(\mathbf{k})+4 n_{0} \hat{v}(\mathbf{k})\right]}
$$

This $E(\mathbf{k})$ is the (approximate) "single-particle excitation energy". When $\hat{h}(\mathbf{k})=\frac{\mathbf{k}^{2}}{2 m}$,

$$
E(\mathbf{k}) \approx c|\mathbf{k}| \text { with } c=\sqrt{\frac{2 n_{0} \hat{v}(0)}{m}} \text { when } \quad \mathbf{k} \approx 0
$$

This "linear dispersion relation" is used, because of the Landau theory of superfluidity, as a signal that the interacting Bose gas is superfluid. The ideal Bose gas has a quadratic dispersion relation and is not superfluid.

The above discussion suggests that there will be a phase transitiion. For $\mu$ below some critical value (which will probably not be exactly zero, because of renormalization 
effects) the expected value of a single annihilation or creation operator will be zero, just as you would expect from conservation of particle number. But, when the temperature is low enough, for $\mu$ above the critical point, it will be $\Phi$ for some complex number of

modulus $|\Phi| \approx \sqrt{\frac{\mu}{2 \hat{v}(0)}} \neq 0$ (despite an action which conserves particle number) and its precise value (i.e. which allowed $\Phi$ it is) will depend on the limiting process used to define the model. So we have to be very careful about how we define the model.

\subsubsection{A Rigorous Starting Point}

To carefully define the model, for example to carefully define the partition function on the left hand side of (1.1), you take a limit of obviously well-defined approximations. One way to get a (pretty) obviously well-defined approximate partition function is to replace space, $\mathbb{R}^{3}$, by a finite number of points, say $X=\mathbb{Z}^{3} / L \mathbb{Z}^{3}$. However, even for an approximate model with space having only a finite number of points, the functional integral on the right hand side of the corresponding (1.1) still has uncountably many integration variables, because time is still $\left(0, \frac{1}{k T}\right]$, and so is still not really defined.

At this point, I am just going to quote a theorem (I'll give the important parts of the proof in §1.4) which says that, when $X$ is finite, you can get a rigorous representation of the partition function by taking a limit of a sequence of integrals, with each integral in the sequence having only finitely many integration variables. To get finitely many integration variables, you replace "time", $\left(0, \frac{1}{k T}\right]$, by a finite number of points too. The theorem, proven in (Balaban et al., 2008b, Theorem 2.2) is the following.

Theorem 1.1 Suppose that $\mathrm{R}(\varepsilon), \mathrm{r}(\varepsilon) \rightarrow \infty$ as $\varepsilon \rightarrow 0$ at suitable rates ${ }^{2}$. For each fixed finite $X$,

$$
\operatorname{Tr} e^{-\frac{1}{k T}(H-\mu N)}=\lim _{\varepsilon \rightarrow 0} \int \prod_{\tau \in \varepsilon \mathbb{Z} \cap\left(0, \frac{1}{k T}\right]}\left[d \mu_{\mathrm{R}(\varepsilon)}\left(\alpha_{\tau}^{*}, \alpha_{\tau}\right) I_{0}\left(\varepsilon ; \alpha_{\tau-\varepsilon}^{*}, \alpha_{\tau}\right)\right]
$$

with the convention that $\alpha_{0}=\alpha_{\frac{1}{k T}}$. Here,

$$
d \mu_{\mathrm{R}(\varepsilon)}\left(\alpha^{*}, \alpha\right)=\prod_{\mathbf{x} \in X} \frac{d \alpha^{*}(\mathbf{x}) \wedge d \alpha(\mathbf{x})}{2 \pi \imath} e^{-\alpha^{*}(\mathbf{x}) \alpha(\mathbf{x})} \chi(|\alpha(\mathbf{x})|<\mathrm{R}(\varepsilon))
$$

denotes an unnormalised Gaussian measure, cut off at radius $\mathrm{R}(\varepsilon)$, and

$$
I_{0}\left(\varepsilon ; \alpha^{*}, \beta\right)=\zeta_{\varepsilon}(\alpha, \beta) e^{\left\langle\alpha^{*}, j(\varepsilon) \beta\right\rangle-\varepsilon\left\langle\alpha^{*} \beta, v \alpha^{*} \beta\right\rangle}
$$

with

$$
j(\varepsilon)=e^{-\varepsilon(h-\mu)}
$$

and $\zeta_{\varepsilon}(\alpha, \beta)$ being the characteristic function of

$$
\left\{\alpha, \beta: \mathbb{C}^{X} \rightarrow \mathbb{C} \mid\|\alpha-\beta\|_{\infty}<\mathrm{r}(\varepsilon)\right\}
$$

We write the $\left(\mathbb{R}\right.$-style) scalar product ${ }^{3},\langle f, g\rangle=\sum_{\mathbf{x} \in X} f(\mathbf{x}) g(\mathbf{x})$ for any two fields $f, g: X \rightarrow \mathbb{C}$.

\footnotetext{
${ }^{2}$ One can think of $\mathrm{R}(\varepsilon) \sim \frac{1}{\sqrt[4]{\varepsilon}}$ and of $\mathrm{r}(\varepsilon)$ as a power of $\ln \frac{1}{\varepsilon}$ or as a small power of $\frac{1}{\varepsilon}$.
}

${ }^{3}$ Thus the usual scalar product over $\mathbb{C}^{|X|}$ is $\left\langle f^{*}, g\right\rangle$. 


\section{The Temporal Ultraviolet Limit}

Now the integrals in this theorem do not look very much like the functional integral on the right hand side of (1.1). In fact, one has a lot of freedom in choosing the integrand in (1.8) and I have deliberately chosen the integrand to make the next steps easy, rather than to make it look like the integrand of (1.1). Here is how to see that the integral of (1.8) is actually not so different from the integral of (1.1).

- First observe that (1.8) has one complex integration variable for each "spacetime" point $(\mathbf{x}, \tau)$ with $\mathbf{x} \in X$ and $\tau \in \varepsilon \mathbb{Z} \cap\left(0, \frac{1}{k T}\right]$, a finite approximation to the "time set" $\left(0, \frac{1}{k T}\right]$.

- In contrast to the integration variables of (1.1), each complex integration variable of (1.8) does not run over all $\mathbb{C}$, because of the cutoff functions $\chi(|\alpha(\mathbf{x})|<\mathrm{R}(\varepsilon))$, which restrict each integration variable to a finite disk in $\mathbb{C}$, and $\zeta_{\varepsilon}\left(\alpha_{\tau-\varepsilon}, \alpha_{\tau}\right)$, which restricts the time-derivative of $\alpha_{\tau}(\mathbf{x})$. But in the limit $\varepsilon \rightarrow 0$, these cutoffs disappear.

- Consider the total exponent

$$
-\sum_{\substack{\mathbf{x} \in X \\ \tau \in \mathbb{Z} \cap\left(0, \frac{1}{k T}\right]}} \alpha_{\tau}(\mathbf{x})^{*} \alpha_{\tau}(\mathbf{x})+\sum_{\tau \in \varepsilon \mathbb{Z} \cap\left(0, \frac{1}{k T}\right]}\left[\left\langle\alpha_{\tau-\varepsilon}^{*}, e^{-\varepsilon(h-\mu)} \alpha_{\tau}\right\rangle-\varepsilon\left\langle\alpha_{\tau-\varepsilon}^{*} \alpha_{\tau}, v \alpha_{\tau-\varepsilon}^{*} \alpha_{\tau}\right\rangle\right]
$$

of (1.8) (including the part of the exponent hidden inside the measure $\left.d \mu_{\mathrm{R}(\varepsilon)}\right)$. Expand the exponential in powers of $\varepsilon$, keeping only $\mathbb{1}-\varepsilon(h-\mu)$ and throwing away all contributions of order at least $\varepsilon^{2}$. This gives exactly

$$
\varepsilon \sum_{\tau \in \varepsilon \mathbb{Z} \cap\left(0, \frac{1}{k T}\right]}\left[\left\langle\alpha_{\tau-\varepsilon}^{*}, \frac{\alpha_{\tau}-\alpha_{\tau-\varepsilon}}{\varepsilon}\right\rangle-\left\langle\alpha_{\tau-\varepsilon}^{*},(h-\mu) \alpha_{\tau}\right\rangle-\left\langle\alpha_{\tau-\varepsilon}^{*} \alpha_{\tau}, v \alpha_{\tau-\varepsilon}^{*} \alpha_{\tau}\right\rangle\right]
$$

In the limit $\varepsilon \rightarrow 0, \varepsilon \sum_{\tau \in \varepsilon \mathbb{Z} \cap\left(0, \frac{1}{k T}\right]}$ becomes $\int_{0}^{\frac{1}{k T}} d \tau$ and $\frac{\alpha_{\tau}-\alpha_{\tau-\varepsilon}}{\varepsilon}$ becomes $\frac{\partial}{\partial \tau} \alpha_{\tau}$ and we get $\mathcal{A}\left(\alpha^{*}, \alpha\right)$.

To get from the integral

$$
\int \prod_{\tau \in \varepsilon \mathbb{Z} \cap\left(0, \frac{1}{k T}\right]}\left[d \mu_{\mathrm{R}(\varepsilon)}\left(\alpha_{\tau}^{*}, \alpha_{\tau}\right) I_{0}\left(\varepsilon ; \alpha_{\tau-\varepsilon}^{*}, \alpha_{\tau}\right)\right]
$$

of the rigorous starting point, (1.8), to the full construction and analysis of the model of interest, we still need to execute several steps.

- Step 1: Take the temporal ultraviolet limit, $\varepsilon \rightarrow 0$. Of course Theorem 1.1 tells us that the limit exists and tells us that it is the approximate partition function. But that information by itself is virtually useless. We need to develop a picture of the limiting value we can work with in later steps.

- Step 2: Take the spatial infrared limit (i.e. the thermodynamic limit) $X \rightarrow \mathbb{Z}^{3}$ and possibly the temporal infrared limit $\frac{1}{k T} \rightarrow \infty$ (i.e. $T \rightarrow 0$ ).

- Step 3: Get properties of the limit, like symmetry breaking.

In these notes, we shall just discuss Step 1, the temporal ultraviolet limit. That is only an extremely small part of full construction. In fact, steps 2 and 3 can be expected to be exceptionally long and arduous and research on them has barely begun. 
Nonetheless, Step 1 is not only a necessary step, but its treatment provides a useful glimpse, in a relatively simple setting, at techniques that are suitable for the later steps, and other models, as well. For a different, earlier, treatment of the ultraviolet limit in some related models see (Ginibre, 1965; Ginibre, 1971; Brydges and Federbush, 1976; Brydges and Federbush, 1977).

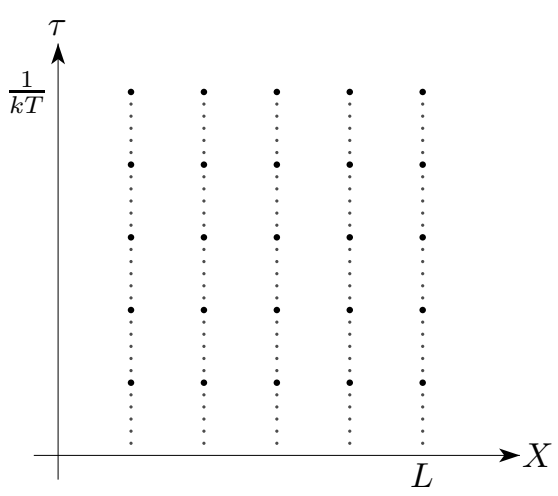

Fig. 1.2 The Integration Variables

In the initial integral, (1.9), there is one complex integration variable, $\alpha_{\tau}(\mathbf{x})$, for each "space-time" point $(\mathbf{x}, \tau)$ with $\mathbf{x} \in X$ and $\tau \in \varepsilon \mathbb{Z} \cap\left(0, \frac{1}{k T}\right]$. Recall that $X$ is the finite discrete torus $\mathbb{Z}^{3} /(L \mathbb{Z})^{3}$, for some large $L \in \mathbb{N}$. Figure 1.2 contains one dot for each of the integration variable labels, $(\mathbf{x}, \tau)$. (Ignore the the difference between light and dark dots for a minute.) You will notice an asymmetry in that figure - the distance, $\varepsilon$, between dots in the $\tau$ direction is miniscule compared to the distance, 1 , between dots in the $X$ direction. In Step 1, we eliminate that asymmetry. We shall "integrate out" all integration variables $\alpha_{\tau}(\mathbf{x})$ for which $(\mathbf{x}, \tau)$ is located at one of the lighter dots in Figure 1.2, leaving the integration variables $\alpha_{\tau}(\mathbf{x})$ for which $(\mathbf{x}, \tau)$ is located at one of the darker dots. That is, the final result for Step 1 is a representation of the partition function as an integral having $\alpha_{\tau}$ as an integration variable only if $\tau \in \theta \mathbb{Z}$ where $\theta$ is some fixed constant, independent of $\varepsilon$. Thus the set of integration variables for the final result of Step 1 looks like the set of integration variables for a classical spin system (in four dimensions). In fact, the final result of Step 1 looks somewhat like the classical $N$-vector spin system for which Balaban proved the existence of the infrared limit and of symmetry breaking in (Balaban 1995a, 1995b, 1996a, $1996 b, 1996 c, 1998 a, 1998 b, 1998 c)$. However there are substantial technical differences between the output of Step 1 and the class of models that Balaban considered. So one cannot execute Steps 2 and 3 simply by saying "Balaban already did it".

To execute Step 1, we repeatedly apply a simple version of a renormalization group procedure, called "decimation". In each decimation step we integrate out all $\alpha_{\tau}$ 's having every second remaining value of $\tau$. In the first decimation step, we integrate out $\alpha_{\tau^{\prime}}$ with $\tau^{\prime}=\varepsilon, 3 \varepsilon, 5 \varepsilon, \cdots$. The integral with respect to these variables factorizes into the product, over $\tau=2 \varepsilon, 4 \varepsilon, 6 \varepsilon, \cdots$, of the independent integrals

$$
\int d \mu_{\mathrm{R}(\varepsilon)}\left(\alpha_{\tau-\varepsilon}^{*}, \alpha_{\tau-\varepsilon}\right) I_{0}\left(\varepsilon ; \alpha_{\tau-2 \varepsilon}^{*}, \alpha_{\tau-\varepsilon}\right) I_{0}\left(\varepsilon ; \alpha_{\tau-\varepsilon}^{*}, \alpha_{\tau}\right)
$$




\section{The Temporal Ultraviolet Limit}

That is, assuming that $\frac{1}{k T} \in 2 \varepsilon \mathbb{N}$,

$$
\begin{aligned}
\int \prod_{\tau \in \varepsilon \mathbb{Z} \cap\left(0, \frac{1}{k T}\right]}\left[d \mu_{\mathrm{R}(\varepsilon)}\left(\alpha_{\tau}^{*}, \alpha_{\tau}\right) I_{0}\left(\varepsilon ; \alpha_{\tau-\varepsilon}^{*}, \alpha_{\tau}\right)\right] \\
=\int \prod_{\tau \in 2 \varepsilon \mathbb{Z} \cap\left(0, \frac{1}{k T}\right]}\left[d \mu_{\mathrm{R}(\varepsilon)}\left(\alpha_{\tau}^{*}, \alpha_{\tau}\right) I_{1}\left(\varepsilon ; \alpha_{\tau-2 \varepsilon}^{*}, \alpha_{\tau}\right)\right]
\end{aligned}
$$

where

$$
I_{1}\left(\varepsilon ; \alpha_{\tau-2 \varepsilon}^{*}, \alpha_{\tau}\right)=\int d \mu_{\mathrm{R}(\varepsilon)}\left(\alpha_{\tau-\varepsilon}^{*}, \alpha_{\tau-\varepsilon}\right) I_{0}\left(\varepsilon ; \alpha_{\tau-2 \varepsilon}^{*}, \alpha_{\tau-\varepsilon}\right) I_{0}\left(\varepsilon ; \alpha_{\tau-\varepsilon}^{*}, \alpha_{\tau}\right)
$$

In the second decimation step, we integrate out $\alpha_{\tau^{\prime}}$ with $\tau^{\prime}=2 \varepsilon, 6 \varepsilon, 10 \varepsilon, \cdots$ in the integral on the right hand side of (1.10). The integral with respect to these variables factorizes into the product, over $\tau=4 \varepsilon, 8 \varepsilon, 12 \varepsilon, \cdots$, of the independent integrals

$$
\int d \mu_{\mathrm{R}(\varepsilon)}\left(\alpha_{\tau-2 \varepsilon}^{*}, \alpha_{\tau-2 \varepsilon}\right) I_{1}\left(\varepsilon ; \alpha_{\tau-4 \varepsilon}^{*}, \alpha_{\tau-2 \varepsilon}\right) I_{1}\left(\varepsilon ; \alpha_{\tau-2 \varepsilon}^{*}, \alpha_{\tau}\right)
$$

That is, assuming that $\frac{1}{k T} \in 4 \varepsilon \mathbb{N}$,

$$
\begin{aligned}
\int \prod_{\tau \in \varepsilon \mathbb{Z} \cap\left(0, \frac{1}{k T}\right]}\left[d \mu_{\mathrm{R}(\varepsilon)}\left(\alpha_{\tau}^{*}, \alpha_{\tau}\right) I_{0}\left(\varepsilon ; \alpha_{\tau-\varepsilon}^{*}, \alpha_{\tau}\right)\right] \\
=\int \prod_{\tau \in 2 \varepsilon \mathbb{Z} \cap\left(0, \frac{1}{k T}\right]}\left[d \mu_{\mathrm{R}(\varepsilon)}\left(\alpha_{\tau}^{*}, \alpha_{\tau}\right) I_{1}\left(\varepsilon ; \alpha_{\tau-2 \varepsilon}^{*}, \alpha_{\tau}\right)\right] \\
=\int \prod_{\tau \in 4 \varepsilon \mathbb{Z} \cap\left(0, \frac{1}{k T}\right]}\left[d \mu_{\mathrm{R}(\varepsilon)}\left(\alpha_{\tau}^{*}, \alpha_{\tau}\right) I_{2}\left(\varepsilon ; \alpha_{\tau-4 \varepsilon}^{*}, \alpha_{\tau}\right)\right]
\end{aligned}
$$

where

$$
\begin{aligned}
I_{2}\left(\varepsilon ; \alpha_{\tau-4 \varepsilon}^{*}, \alpha_{\tau}\right) & =\int d \mu_{\mathrm{R}(\varepsilon)}\left(\alpha_{\tau-2 \varepsilon}^{*}, \alpha_{\tau-2 \varepsilon}\right) I_{1}\left(\varepsilon ; \alpha_{\tau-4 \varepsilon}^{*}, \alpha_{\tau-2 \varepsilon}\right) I_{1}\left(\varepsilon ; \alpha_{\tau-2 \varepsilon}^{*}, \alpha_{\tau}\right) \\
& =\int \prod_{\tau^{\prime} \in \varepsilon \mathbb{Z} \cap(\tau-4 \varepsilon, \tau)} d \mu_{\mathrm{R}(\varepsilon)}\left(\alpha_{\tau^{\prime}}^{*}, \alpha_{\tau^{\prime}}\right) \prod_{\tau \in \varepsilon \mathbb{Z} \cap(\tau-4 \varepsilon, \tau]} I_{0}\left(\varepsilon ; \alpha_{\tau^{\prime}-\varepsilon}^{*}, \alpha_{\tau^{\prime}}\right)
\end{aligned}
$$

In general, for $n \geq 1, \varepsilon>0$, set

$$
I_{n}\left(\varepsilon ; \alpha^{*}, \beta\right)=\int \prod_{\tau \in \varepsilon \mathbb{Z} \cap\left(0,2^{n} \varepsilon\right)} d \mu_{\mathrm{R}(\varepsilon)}\left(\alpha_{\tau}^{*}, \alpha_{\tau}\right) \prod_{\tau \in \varepsilon \mathbb{Z} \cap\left(0,2^{n} \varepsilon\right]} I_{0}\left(\varepsilon ; \alpha_{\tau-\varepsilon}^{*}, \alpha_{\tau}\right)
$$

with $\alpha_{0}=\alpha$ and $\alpha_{2^{n} \varepsilon}=\beta$. If, as in Figure 1.3 , below, $\frac{1}{k T}=p \theta$ and $\varepsilon=2^{-m} \theta$, then

$$
\int \prod_{\tau \in \varepsilon \mathbb{Z} \cap\left(0, \frac{1}{k T}\right]}\left[d \mu_{\mathrm{R}(\varepsilon)}\left(\alpha_{\tau}^{*}, \alpha_{\tau}\right) I_{0}\left(\varepsilon ; \alpha_{\tau-\varepsilon}^{*}, \alpha_{\tau}\right)\right]=\int \prod_{\ell=1}^{p}\left[d \mu_{\mathrm{R}(\varepsilon)}\left(\phi_{\ell}^{*}, \phi_{\ell}\right) I_{m}\left(\varepsilon ; \phi_{\ell-1}^{*}, \phi_{\ell}\right)\right]
$$

with the convention $\phi_{0}=\phi_{p}$. I have renamed $\alpha_{\ell \theta}=\phi_{\ell}$. 


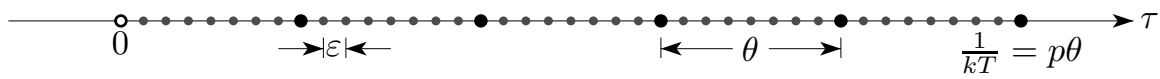

Fig. 1.3 The Integration Variables, Again

Combining (1.8) and (1.13) we get

$$
\operatorname{Tr} e^{-\frac{1}{k T}(H-\mu N)}=\lim _{m \rightarrow \infty} \int \prod_{\ell=1}^{p}\left[d \mu_{\mathrm{R}\left(2^{-m} \theta\right)}\left(\phi_{\ell}^{*}, \phi_{\ell}\right) I_{m}\left(2^{-m} \theta ; \phi_{\ell-1}^{*}, \phi_{\ell}\right)\right]
$$

So far we have just made a trivial rearrangement of the order of integration. But ... (Balaban et al., 2010c) have shown that

- $I_{\theta}\left(\alpha^{*}, \beta\right)=\lim _{m \rightarrow \infty} I_{m}\left(2^{-m} \theta ; \alpha^{*}, \beta\right)$ exists

- and that the partition function can be written as

$$
\operatorname{Tr} e^{-\frac{1}{k T}(H-\mu N)}=\int \prod_{\ell=1}^{p}\left[\prod_{\mathbf{x} \in X} \frac{d \phi_{\ell}(\mathbf{x})^{*} \phi_{\ell}(\mathbf{x})}{2 \pi \imath} e^{-\phi_{\ell}(\mathbf{x})^{*} \phi_{\ell}(\mathbf{x})}\right] I_{\theta}\left(\phi_{\ell-1}^{*}, \phi_{\ell}\right)
$$

- and that, if $\theta$ was chosen sufficiently small, $I_{\theta}$ may be written as the sum of a dominant part (which is shown to have a logarithm, which I will describe in more detail below) plus (ugly) terms indexed by proper subsets of $X$ and which are nonperturbatively small, exponentially in the size of the subsets.

We call the dominant term the "stationary phase approximation" (SP), because it is obtained by restricting all domains of integration in our functional integrals, simply by fiat, to appropriate neighbourhoods of stationary points. I'll describe this process in more detail in $\S 1.2$. The dominant contribution looks just like a perturbation of the original $e^{\left\langle\alpha^{*}, j(\varepsilon) \beta\right\rangle-\varepsilon\left\langle\alpha^{*} \beta, v \alpha^{*} \beta\right\rangle}$ in our starting point (1.8). Here is the precise form of the dominant contribution to $I_{n}\left(\varepsilon ; \alpha^{*}, \beta\right)$.

$$
I_{n}^{(\mathrm{SP})}\left(\varepsilon ; \alpha^{*}, \beta\right)=\mathcal{Z}_{2^{n} \varepsilon}(\varepsilon)^{|X|} e^{\left\langle\alpha^{*}, j\left(2^{n} \varepsilon\right) \beta\right\rangle+\mathcal{V}_{2^{n}}\left(\varepsilon ; \alpha^{*}, \beta\right)+\mathcal{E}_{2^{n} \varepsilon}\left(\varepsilon ; \alpha^{*}, \beta\right)}
$$

where, for every $\delta$ that is an integer multiple of $\varepsilon$,

$$
\mathcal{V}_{\delta}\left(\varepsilon ; \alpha^{*}, \beta\right)=-\varepsilon \sum_{\tau \in \varepsilon \mathbb{Z} \cap[0, \delta)}\left\langle\left[j(\tau) \alpha^{*}\right][j(\delta-\tau-\varepsilon) \beta], v\left[j(\tau) \alpha^{*}\right][j(\delta-\tau-\varepsilon) \beta]\right\rangle
$$

The normalization constant $\mathcal{Z}_{\delta}(\varepsilon)$ is chosen so that $\mathcal{E}_{\delta}(\varepsilon ; 0,0)=0$. It is extremely close to 1. (See (Balaban et al., 2010b, Appendix C).) The function $\mathcal{E}_{\delta}\left(\varepsilon ; \alpha^{*}, \beta\right)$ is defined for real numbers $0<\varepsilon \leq \delta \leq \Theta$ such that $\delta=2^{n} \varepsilon$ for some integer $n \geq 0$. It is determined by the recursion relation

$$
\begin{aligned}
& \mathcal{E}_{\varepsilon}\left(\varepsilon ; \alpha^{*}, \beta\right)=0 \\
& \mathcal{E}_{2 \delta}\left(\varepsilon ; \alpha^{*}, \beta\right)=\mathcal{E}_{\delta}\left(\varepsilon ; \alpha^{*}, j(\delta) \beta\right) \\
&+\mathcal{E}_{\delta}\left(\varepsilon ; j(\delta) \alpha^{*}, \beta\right) \\
&+\log \frac{\int d \mu_{\mathrm{r}(\delta)}\left(z^{*}, z\right) e^{\partial \mathcal{A}_{\delta}\left(\varepsilon ; \alpha^{*}, \beta ; z^{*}, z\right)}}{\int d \mu_{\mathrm{r}(\delta)}\left(z^{*}, z\right)}
\end{aligned}
$$

where 


$$
\begin{aligned}
\partial \mathcal{A}_{\delta}\left(\varepsilon ; \alpha^{*}, \beta ; z_{*}, z\right)= & {\left[\mathcal{V}_{\delta}\left(\varepsilon ; \alpha^{*}, j(\delta) \beta+z\right)-\mathcal{V}_{\delta}\left(\varepsilon ; \alpha^{*}, j(\delta) \beta\right)\right] } \\
& +\left[\mathcal{V}_{\delta}\left(\varepsilon ; j(\delta) \alpha^{*}+z_{*}, \beta\right)-\mathcal{V}_{\delta}\left(\varepsilon ; j(\delta) \alpha^{*}, \beta\right)\right] \\
& +\left[\mathcal{E}_{\delta}\left(\varepsilon ; \alpha^{*}, j(\delta) \beta+z\right)-\mathcal{E}_{\delta}\left(\varepsilon ; \alpha^{*}, j(\delta) \beta\right)\right] \\
& +\left[\mathcal{E}_{\delta}\left(\varepsilon ; j(\delta) \alpha^{*}+z_{*}, \beta\right)-\mathcal{E}_{\delta}\left(\varepsilon ; j(\delta) \alpha^{*}, \beta\right)\right]
\end{aligned}
$$

The motivation for this recursion relation comes from the stationary phase construction and is given in $\S 1.2$. In $\S 1.3$, I will outline the argument that the functions $\mathcal{E}_{\delta}\left(\varepsilon ; \alpha^{*}, \beta\right)$ are

- analytic function of the fields,

- of degree at least two in each of $\alpha^{*}$ and $\beta$

- perturbatively small corrections

\subsection{Motivation for the Stationary Phase Approximation}

The functions $I_{n}\left(\varepsilon ; \alpha^{*}, \beta\right)$ of (1.12) can also be defined recursively by

$$
I_{n+1}\left(\varepsilon ; \alpha^{*}, \beta\right)=\int d \mu_{\mathrm{R}(\varepsilon)}\left(\phi^{*}, \phi\right) I_{n}\left(\varepsilon ; \alpha^{*}, \phi\right) I_{n}\left(\varepsilon ; \phi^{*}, \beta\right)
$$

One of the morals of (Balaban et al., 2010c) is that the integrand is highly oscillatory and that the dominant contributions may be extracted using stationary phase by discarding contributions far away from the critical point of the ("free part") of the exponent.

By way of motivation for the stationary phase approximation, and in particular for the recursive definition $(1.16)$ of $\mathcal{E}_{\delta}\left(\varepsilon ; \alpha^{*}, \beta\right)$, replace $I_{n}$ by

$$
I_{n}^{(\mathrm{SP})}\left(\varepsilon ; \alpha^{*}, \beta\right)=\mathcal{Z}_{\varepsilon_{n}}(\varepsilon)^{|X|} e^{\left\langle\alpha^{*}, j\left(\varepsilon_{n}\right) \beta\right\rangle+\mathcal{V}_{\varepsilon_{n}}\left(\varepsilon ; \alpha^{*}, \beta\right)+\mathcal{E}_{\varepsilon_{n}}\left(\varepsilon ; \alpha^{*}, \beta\right)}
$$

in the recursion relation (1.18). Here, $\varepsilon_{n}=2^{n} \varepsilon$. (Start with $n=0, \mathcal{Z}_{\varepsilon}(\varepsilon)=1$ and $\mathcal{E}_{\varepsilon}\left(\varepsilon ; \alpha^{*}, \beta\right)=0$. Then, aside from the cutoff function $\zeta_{\varepsilon}(\alpha, \beta)$, which is going to incorporated by our choice of domain of integration, $I_{0}^{(\mathrm{SP})}\left(\varepsilon ; \alpha^{*}, \beta\right)$ is the same as $I_{0}\left(\varepsilon ; \alpha^{*}, \beta\right)$.) The resulting integral

$$
\begin{aligned}
& \int d \mu_{\mathrm{R}(\varepsilon)}\left(\phi^{*}, \phi\right) I_{n}^{(\mathrm{SP})}\left(\varepsilon ; \alpha^{*}, \phi\right) I_{n}^{(\mathrm{SP})}\left(\varepsilon ; \phi^{*}, \beta\right) \\
& =\mathcal{Z}_{\varepsilon_{n}}(\varepsilon)^{2|X|} \int d \mu_{\mathrm{R}(\varepsilon)}\left(\phi^{*}, \phi\right) e^{\left\langle\alpha^{*}, j\left(\varepsilon_{n}\right) \phi\right\rangle+\left\langle\phi^{*}, j\left(\varepsilon_{n}\right) \beta\right\rangle} e^{\mathcal{V}_{\varepsilon_{n}}\left(\varepsilon ; \alpha^{*}, \phi\right)+\mathcal{V}_{\varepsilon_{n}}\left(\varepsilon ; \phi^{*}, \beta\right)} \\
& e^{\mathcal{E}_{\varepsilon_{n}}\left(\varepsilon ; \alpha^{*}, \phi\right)+\mathcal{E}_{\varepsilon_{n}}\left(\varepsilon ; \phi^{*}, \beta\right)} \\
& =\mathcal{Z}_{\varepsilon_{n}}(\varepsilon)^{2|X|}\left[\prod_{\mathbf{x} \in X} \int_{|\phi(\mathbf{x})|<\mathrm{R}(\varepsilon)} \frac{d \phi^{*}(\mathbf{x}) \wedge d \phi(\mathbf{x})}{2 \pi \imath}\right] e^{\mathcal{A}\left(\alpha^{*}, \beta ; \phi^{*}, \phi\right)} \\
& =\mathcal{Z}_{\mathcal{\varepsilon}_{n}}(\varepsilon)^{2|X|}\left[\prod_{\mathbf{x} \in X} \int_{\substack{|\phi(\mathbf{x})|<\mathrm{R}(\varepsilon) \\
\phi(\mathbf{x})=\phi(\mathbf{x})^{*}}} \frac{d \phi_{*}(\mathbf{x}) \wedge d \phi(\mathbf{x})}{2 \pi \imath}\right] e^{\mathcal{A}\left(\alpha^{*}, \beta ; \phi_{*}, \phi\right)}
\end{aligned}
$$

with 


$$
\begin{aligned}
\mathcal{A}\left(\alpha^{*}, \beta ; \phi_{*}, \phi\right)=-\left\langle\phi_{*}\right. & , \phi\rangle+\left\langle\alpha^{*}, j\left(\varepsilon_{n}\right) \phi\right\rangle+\left\langle\phi_{*}, j\left(\varepsilon_{n}\right) \beta\right\rangle \\
& +\mathcal{V}_{\varepsilon_{n}}\left(\varepsilon ; \alpha^{*}, \phi\right)+\mathcal{V}_{\varepsilon_{n}}\left(\varepsilon ; \phi_{*}, \beta\right) \\
& +\mathcal{E}_{\varepsilon_{n}}\left(\varepsilon ; \alpha^{*}, \phi\right)+\mathcal{E}_{\varepsilon_{n}}\left(\varepsilon ; \phi_{*}, \beta\right)
\end{aligned}
$$

Here we have written $\mathcal{A}$ as a function of four independent complex fields $\alpha^{*}, \beta, \phi_{*}$ and $\phi$. The activity in the penultimate line of (1.19) is obtained simply by evaluating $\mathcal{A}\left(\alpha^{*}, \beta ; \phi_{*}, \phi\right)$ with $\phi_{*}=\phi^{*}$, the complex conjugate of $\phi$. But in the last line, we introduce, for each $\mathbf{x} \in X$, a new, complex integration variable $\phi_{*}(\mathbf{x})$. That is, $\left(\phi(\mathbf{x}), \phi_{*}(\mathbf{x})\right) \in \mathbb{C}^{2}$. To get equality between the second last line and the last line of (1.19), we build the condition $\phi_{*}(\mathbf{x})=\phi(\mathbf{x})^{*}$ into the domain of integration. The reason for introducing independent complex fields $\phi_{*}$ and $\phi$ lies in the fact that the critical point (where the first order derivatives with respect to $\phi_{*}$ and $\phi$ vanish) of the quadratic part

$$
\begin{aligned}
-\left\langle\phi_{*}, \phi\right\rangle & +\left\langle j\left(\varepsilon_{n}\right) \alpha^{*}, \phi\right\rangle+\left\langle\phi_{*}, j\left(\varepsilon_{n}\right) \beta\right\rangle \\
& =-\left\langle\phi_{*}-j\left(\varepsilon_{n}\right) \alpha^{*}, \phi-j\left(\varepsilon_{n}\right) \beta\right\rangle+\underbrace{\left\langle j\left(\varepsilon_{n}\right) \alpha^{*}, j\left(\varepsilon_{n}\right) \beta\right\rangle}_{\left\langle\alpha^{*}, j\left(\varepsilon_{n+1}\right) \beta\right\rangle}
\end{aligned}
$$

of $\mathcal{A}$ is "not real". Precisely, the critical point is

$$
\phi_{*}^{\text {crit }}=j\left(\varepsilon_{n}\right) \alpha^{*}, \quad \phi^{\text {crit }}=j\left(\varepsilon_{n}\right) \beta
$$

and in general $\left(\phi_{*}^{\text {crit }}\right)^{*} \neq \phi^{\text {crit }}$. To do stationary phase, we introduce the "fluctuation variables" $z_{*}(\mathbf{x}), z(\mathbf{x})$ and make the change of variables

$$
\phi_{*}(\mathbf{x})=\phi_{*}^{\text {crit }}(\mathbf{x})+z_{*}(\mathbf{x}), \quad \phi(\mathbf{x})=\phi^{\text {crit }}(\mathbf{x})+z(\mathbf{x})
$$

Under this change of variables the domain of integration

$$
\left\{\left(\phi_{*}(\mathbf{x}), \phi(\mathbf{x})\right)\left|\phi_{*}(\mathbf{x})=\phi(\mathbf{x})^{*},\right| \phi(\mathbf{x}) \mid<\mathrm{R}(\varepsilon)\right\}
$$

is transformed into

$$
\begin{aligned}
M(\mathbf{x})=\left\{\left(z_{*}(\mathbf{x}), z(\mathbf{x})\right) \mid\left(\phi_{*}^{\text {crit }}(\mathbf{x})+z_{*}(\mathbf{x})\right)^{*}\right. & =\phi^{\text {crit }}(\mathbf{x})+z(\mathbf{x}) \\
& \text { and } \left.\left|\phi^{\text {crit }}(\mathbf{x})+z(\mathbf{x})\right|<\mathrm{R}(\varepsilon)\right\}
\end{aligned}
$$

After the change of variables, the integral (1.19) is over a real $2|X|$ dimensional subset in the complex $2|X|$ dimensional space of fields $z_{*}, z$.

The first step in the stationary phase approximation is to replace, for each $\mathbf{x} \in X$, the domain of integration $M(\mathbf{x})$ by the neighbourhood

$$
\begin{aligned}
D(\mathbf{x})=\left\{\left(z_{*}(\mathbf{x}), z(\mathbf{x})\right) \in \mathbb{C}^{2} \mid\right. & \left|z_{*}(\mathbf{x})\right| \leq \mathrm{r}\left(\varepsilon_{n}\right),|z(\mathbf{x})| \leq \mathrm{r}\left(\varepsilon_{n}\right), \\
& \left.\left(z_{*}(\mathbf{x})+\phi_{*}^{\text {crit }}(\mathbf{x})\right)^{*}=z(\mathbf{x})+\phi^{\text {crit }}(\mathbf{x})\right\}
\end{aligned}
$$

of the critical point. In (Balaban et al., 2010c) we justify this approximation by the observation that, whenever $\left(z_{*}(\mathbf{x}), z(\mathbf{x})\right) \notin D(\mathbf{x})$ for some $\mathbf{x} \in X$, the integrand is 


\section{The Temporal Ultraviolet Limit}

extremely small. I will sketch the reasons for this in $\$ 1.2 .2$, below. Observe that, in general, first, the critical point $z(\mathbf{x})=z_{*}(\mathbf{x})=0$ is not in $D(\mathbf{x})$, and, second, $z_{*}(\mathbf{x}) \neq z(\mathbf{x})^{*}$ on $D(\mathbf{x})$.

The quadratic part (1.20) of the effective action $\mathcal{A}\left(\alpha^{*}, \beta ; \phi_{*}^{\text {crit }}+z_{*}, \phi^{\text {crit }}+z\right)$ in the new variables is

$$
\begin{gathered}
-\left\langle j\left(\varepsilon_{n}\right) \alpha^{*}+z_{*}, j\left(\varepsilon_{n}\right) \beta+z\right\rangle+\left\langle\alpha^{*}, j\left(\varepsilon_{n}\right)\left(j\left(\varepsilon_{n}\right) \beta+z\right)\right\rangle \\
+\left\langle j\left(\varepsilon_{n}\right)\left(j\left(\varepsilon_{n}\right) \alpha^{*}+z_{*}\right), \beta\right\rangle \\
=-\left\langle z_{*}, z\right\rangle+\left\langle\alpha^{*}, j\left(\varepsilon_{n+1}\right) \beta\right\rangle
\end{gathered}
$$

(This is why we introduced the $j(\varepsilon)$ in Theorem 1.1.) Inserting the change of variables (1.21), we see that the part of (1.19) near the critical point is,

$$
\mathcal{Z}_{\varepsilon_{n}}(\varepsilon)^{2|X|}\left[\prod_{\mathbf{x} \in X} \int_{D(\mathbf{x})} \frac{d z_{*}(\mathbf{x}) \wedge d z(\mathbf{x})}{2 \pi \imath}\right] e^{\tilde{\mathcal{A}}\left(\alpha^{*}, \beta ; z_{*}, z\right)}
$$

where

$$
\begin{aligned}
\tilde{\mathcal{A}}\left(\alpha^{*}, \beta ; z_{*}, z\right)=- & \left\langle z_{*}, z\right\rangle+\left\langle\alpha^{*}, j\left(\varepsilon_{n+1}\right) \beta\right\rangle \\
& +\mathcal{V}_{\varepsilon_{n}}\left(\varepsilon ; \alpha^{*}, \phi^{\text {crit }}+z\right)+\mathcal{V}_{\varepsilon_{n}}\left(\varepsilon ; \phi_{*}^{\text {crit }}+z_{*}, \beta\right) \\
& +\mathcal{E}_{\varepsilon_{n}}\left(\varepsilon ; \alpha^{*}, \phi^{\text {crit }}+z\right)+\mathcal{E}_{\varepsilon_{n}}\left(\varepsilon ; \phi_{*}^{\text {crit }}+z_{*}, \beta\right) \\
=- & \left\langle z_{*}, z\right\rangle+\left\langle\alpha^{*}, j\left(\varepsilon_{n+1}\right) \beta\right\rangle+\mathcal{V}_{\varepsilon_{n+1}}\left(\varepsilon ; \alpha^{*}, \beta\right) \\
& +\mathcal{E}_{\varepsilon_{n}}\left(\varepsilon ; \alpha^{*}, \phi^{\text {crit }}\right)+\mathcal{E}_{\varepsilon_{n}}\left(\varepsilon ; \phi_{*}^{\text {crit }}, \beta\right)+\partial \mathcal{A}_{\varepsilon_{n}}\left(\varepsilon ; \alpha^{*}, \beta ; z_{*}, z\right)
\end{aligned}
$$

with the part of $\tilde{\mathcal{A}}\left(\alpha^{*}, \beta ; z_{*}, z\right)$ that is of degree at least one in $\left(z_{*}, z\right)$ being (except for the explicit $\left.-\left\langle z_{*}, z\right\rangle\right)$

$$
\begin{aligned}
\partial \mathcal{A}_{\delta}\left(\varepsilon ; \alpha^{*}, \beta ; z_{*}, z\right)= & {\left[\mathcal{V}_{\delta}\left(\varepsilon ; \alpha^{*}, j(\delta) \beta+z\right)-\mathcal{V}_{\delta}\left(\varepsilon ; \alpha^{*}, j(\delta) \beta\right)\right] } \\
& +\left[\mathcal{V}_{\delta}\left(\varepsilon ; j(\delta) \alpha^{*}+z_{*}, \beta\right)-\mathcal{V}_{\delta}\left(\varepsilon ; j(\delta) \alpha^{*}, \beta\right)\right] \\
& +\left[\mathcal{E}_{\delta}\left(\varepsilon ; \alpha^{*}, j(\delta) \beta+z\right)-\mathcal{E}_{\delta}\left(\varepsilon ; \alpha^{*}, j(\delta) \beta\right)\right] \\
& +\left[\mathcal{E}_{\delta}\left(\varepsilon ; j(\delta) \alpha^{*}+z_{*}, \beta\right)-\mathcal{E}_{\delta}\left(\varepsilon ; j(\delta) \alpha^{*}, \beta\right)\right]
\end{aligned}
$$

We have used that

$$
\begin{aligned}
\mathcal{V}_{\varepsilon_{n}}\left(\varepsilon ; \alpha^{*}, \phi^{\text {crit }}\right)+\mathcal{V}_{\varepsilon_{n}}\left(\varepsilon ; \phi_{*}^{\text {crit }}, \beta\right) & =\mathcal{V}_{\varepsilon_{n}}\left(\varepsilon ; \alpha^{*}, j\left(\varepsilon_{n}\right) \beta\right)+\mathcal{V}_{\varepsilon_{n}}\left(\varepsilon ; j\left(\varepsilon_{n}\right) \alpha^{*}, \beta\right) \\
& =\mathcal{V}_{\varepsilon_{n+1}}\left(\varepsilon ; \alpha^{*}, \beta\right)
\end{aligned}
$$

(The definition (1.15) of $\mathcal{V}_{\delta}\left(\varepsilon ; \alpha^{*}, \beta\right)$ has been rigged to give this.) Apply Stokes' Theorem, once for each $\mathbf{x} \in X$, to replace the domain $D(\mathbf{x})$ with the union of

$$
\left\{\left(z_{*}(\mathbf{x}), z(\mathbf{x})\right)\left|z_{*}(\mathbf{x})=z(\mathbf{x})^{*},\right| z(\mathbf{x}) \mid \leq \mathrm{r}\left(\varepsilon_{n}\right)\right\}
$$

(which contains the critical point) and a "side boundary". This is done in Lemma 1.2 below. (Choose $r=\mathrm{r}\left(\varepsilon_{n}\right)$ and $\rho(\mathbf{x})=\phi_{*}^{\text {crit }}(\mathbf{x})^{*}-\phi^{\text {crit }}(\mathbf{x})=\left(j\left(\varepsilon_{n}\right)(\alpha-\beta)\right)(\mathbf{x})$.) This gives that (1.23) is the sum of 


$$
\mathcal{Z}_{\varepsilon_{n}}(\varepsilon)^{2|X|}\left[\prod_{\mathbf{x} \in X} \int_{|z(\mathbf{x})| \leq \mathrm{r}\left(\varepsilon_{n}\right)} \frac{d z^{*}(\mathbf{x}) \wedge d z(\mathbf{x})}{2 \pi \imath}\right] e^{\tilde{\mathcal{A}}\left(\alpha^{*}, \beta ; z^{*}, z\right)}
$$

and $\mathcal{Z}_{\varepsilon_{n}}(\varepsilon)^{2|X|}$ times

$$
\left.\sum_{\substack{R \subset X \\ R \neq \emptyset}}\left[\prod_{\mathbf{x} \in R} \int_{C(\mathbf{x})} \frac{d z_{*}(\mathbf{x}) \wedge d z(\mathbf{x})}{2 \pi i}\right]\left[\prod_{\mathbf{x} \in X \backslash R} \int_{|z(\mathbf{x})| \leq \mathrm{r}\left(\varepsilon_{n}\right)} \frac{d z(\mathbf{x})^{*} \wedge d z(\mathbf{x})}{2 \pi i}\right] e^{\tilde{\mathcal{A}}\left(\alpha^{*}, \beta ; z^{*}, z\right)}\right|_{\substack{z *(\mathbf{x})=z(\mathbf{x})^{*} \\ \text { for } \mathbf{x} \in \mathrm{X} \backslash \mathrm{R}}}
$$

where, for each $\mathbf{x} \in X, C(\mathbf{x})$ is a two real dimensional submanifold of $\mathbb{C}^{2}$ whose boundary is the union of "circles" $\partial D(\mathbf{x})$ and

$$
\left\{\left(z_{*}(\mathbf{x}), z(\mathbf{x})\right) \in \mathbb{C}^{2}\left|z_{*}^{*}(\mathbf{x})=z(\mathbf{x}),\right| z(\mathbf{x}) \mid=\mathrm{r}\left(\varepsilon_{n}\right)\right\}
$$

The second step in the stationary phase approximation is to ignore all but the first term. That is, to replace (1.23) with (1.24). In (Balaban et al., 2010c) we argue that $-z_{*}(\mathbf{x}) z(\mathbf{x})$ has an extremely large negative real part whenever $\left(z_{*}(\mathbf{x}), z(\mathbf{x})\right) \in C(\mathbf{x})$ (see part (b) of Lemma 1.2, below) and that this replacement introduces a nonperturbatively small error.

Thus, the stationary phase approximation for

$$
\int d \mu_{\mathrm{R}(\varepsilon)}\left(\phi^{*}, \phi\right) I_{n}^{(\mathrm{SP})}\left(\varepsilon ; \alpha^{*}, \phi\right) I_{n}^{(\mathrm{SP})}\left(\varepsilon ; \phi^{*}, \beta\right)
$$

is (1.24), which can also be written as

$$
\begin{aligned}
\mathcal{Z}_{\varepsilon_{n}}(\varepsilon)^{2|X|} & e^{\left\langle\alpha^{*}, j\left(\varepsilon_{n+1}\right) \beta\right\rangle+\mathcal{V}_{\varepsilon_{n+1}}\left(\varepsilon ; \alpha^{*}, \beta\right)} \\
& e^{\mathcal{E}_{\varepsilon_{n}}\left(\varepsilon ; \alpha^{*}, j\left(\varepsilon_{n}\right) \beta\right)+\mathcal{E}_{\varepsilon_{n}}\left(\varepsilon ; j\left(\varepsilon_{n}\right) \alpha^{*}, \beta\right)} \int d \mu_{\mathrm{r}\left(\varepsilon_{n}\right)}\left(z^{*}, z\right) e^{\partial \mathcal{A}_{\varepsilon_{n}}\left(\varepsilon ; \alpha^{*}, \beta ; z_{*}, z\right)}
\end{aligned}
$$

This is indeed of the desired form, namely (1.14) with $n$ replaced by $n+1$, if

$$
\mathcal{Z}_{\varepsilon_{n+1}}(\varepsilon)=\mathcal{Z}_{\varepsilon_{n}}(\varepsilon)^{2} \int_{|z|<\mathrm{r}\left(\varepsilon_{n}\right)} \frac{d z^{*} \wedge d z}{2 \pi i} e^{-|z|^{2}}
$$

and $\mathcal{E}_{\varepsilon_{n+1}}\left(\varepsilon ; \alpha^{*}, \beta\right)$ is given by the recursion relation (1.16).

\subsubsection{Stokes' Theorem}

We next give a short discussion and proof of the version of Stokes' Theorem that we used above. The setting is that we are given a radius $r>0$ and a complex vector $\rho \in \mathbb{C}^{X}$ that obeys $|\rho(\mathbf{x})|<2 r$ for all $\mathbf{x} \in X$ and we wish to "move the domain of integration" from the initial domain $D_{\mathbb{C}}=\underset{\mathbf{x} \in X}{\chi_{\mathbb{C}}} D_{\mathbb{C}}(\mathbf{x})$, where

$$
D_{\mathbb{C}}(\mathbf{x})=\left\{\left(z_{*}(\mathbf{x}), z(\mathbf{x})\right) \in \mathbb{C}^{2}|| z_{*}(\mathbf{x})|\leq r,| z(\mathbf{x}) \mid \leq r, z(\mathbf{x})-z_{*}(\mathbf{x})^{*}=\rho(\mathbf{x})\right\}
$$

(see $(1.22)$ above) to the final domain $\left.D_{\mathbb{R}}=\underset{\mathbf{x} \in X}{\chi_{\mathbb{R}}} D_{\mathbb{x}}\right)$, where

$$
D_{\mathbb{R}}(\mathbf{x})=\left\{\left(z_{*}(\mathbf{x}), z(\mathbf{x})\right) \in \mathbb{C}^{2}\left|z_{*}^{*}(\mathbf{x})=z(\mathbf{x}),\right| z(\mathbf{x}) \mid \leq r\right\}
$$

We start by taking a closer look at $D_{\mathbb{C}}(\mathbf{x})$. At each point of $D_{\mathbb{C}}(\mathbf{x})$, the value of the variable $z_{*}(\mathbf{x})$ is completely determined by the value of the variable $z(\mathbf{x})$ through 


\section{The Temporal Ultraviolet Limit}

$z_{*}(\mathbf{x})=z(\mathbf{x})^{*}-\rho(\mathbf{x})^{*}$. The set of allowed values of the variable $z(\mathbf{x})$ is precisely the intersection of the two discs $|z(\mathbf{x})| \leq r$ and $|z(\mathbf{x})-\rho(\mathbf{x})| \leq r$. The two discs overlap because of the hypothesis $|\rho(\mathbf{x})|<2 r$. At each point of the corresponding final domain $D_{\mathbb{R}}(\mathbf{x})$, the value of the variable $z_{*}(\mathbf{x})$ is again completely determined by the value of the variable $z(\mathbf{x})$, through $z_{*}(\mathbf{x})=z(\mathbf{x})^{*}$, and the set of allowed values of the variable $z(\mathbf{x})$ can be though of as being precisely the intersection of the two discs $|z(\mathbf{x})| \leq r$ and $|z(\mathbf{x})-0| \leq r$, which happen to coincide.

It is a simple matter to interpolate between $D_{\mathbb{C}}(\mathbf{x})$ and $D_{\mathbb{R}}(\mathbf{x})$. Define, for each $0 \leq t \leq 1$,

$$
D_{t}(\mathbf{x})=\left\{\left(z_{*}(\mathbf{x}), z(\mathbf{x})\right) \in \mathbb{C}^{2}|| z_{*}(\mathbf{x})|\leq r,| z(\mathbf{x}) \mid \leq r, z(\mathbf{x})-z_{*}(\mathbf{x})^{*}=t \rho(\mathbf{x})\right\}
$$

Once again, at each point of $D_{t}(\mathbf{x})$, the value of the variable $z_{*}(\mathbf{x})$ is completely determined by the value of the variable $z(\mathbf{x})$, this time through $z_{*}(\mathbf{x})=z(\mathbf{x})^{*}-t \rho(\mathbf{x})^{*}$, and the set of allowed values of the variable $z(\mathbf{x})$ is precisely the intersection of the two discs $|z(\mathbf{x})| \leq r$ and $|z(\mathbf{x})-t \rho(\mathbf{x})| \leq r$. When $t=1, D_{t}(\mathbf{x})=D_{\mathbb{C}}(\mathbf{x})$ and when $t=0, D_{t}(\mathbf{x})=D_{\mathbb{R}}(\mathbf{x})$. Hence $\mathcal{B}(\mathbf{x})=\bigcup_{0 \leq t \leq 1} D_{t}(\mathbf{x})$ is a the three (real) dimensional set

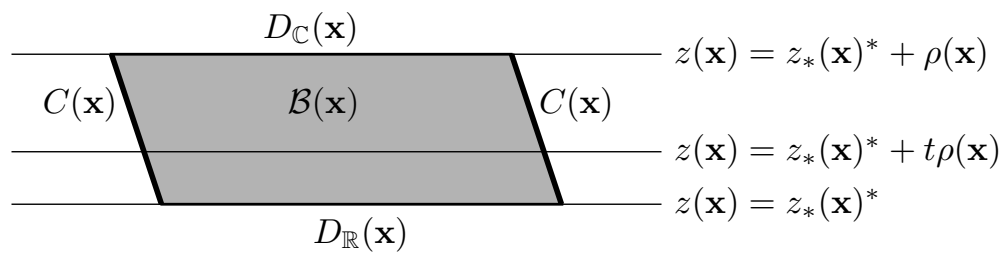

Fig. 1.4 The domain of integration for Stokes' Theorem

whose boundary is the union of $D_{\mathbb{C}}(\mathbf{x})$ (that's the part of the boundary with $t=1$ ) and $D_{\mathbb{R}}(\mathbf{x})$ (that's the part of the boundary with $t=0$ ) and the two (real) dimensional surface $C(\mathbf{x})=\bigcup_{0<t<1} \partial D_{t}(\mathbf{x})$ (that's the part of the boundary with $0<t<1$ ) where

$$
\partial D_{t}(\mathbf{x})=\left\{\left(z_{*}(\mathbf{x}), z(\mathbf{x})\right) \in \mathbb{C}^{2} \mid \max \left\{\left|z_{*}(\mathbf{x})\right|,|z(\mathbf{x})|\right\}=r, z(\mathbf{x})-z_{*}(\mathbf{x})^{*}=t \rho(\mathbf{x})\right\}
$$

The surface $C(\mathbf{x})$ the joins the curves bounding $D_{\mathbb{R}}(\mathbf{x})$ and $D_{\mathbb{C}}(\mathbf{x})$.

Lemma 1.2 (a) Let $f\left(\alpha_{*}, \beta ; z_{*}, z\right)$ be a function that is analytic in the variables $\alpha_{*}, \beta$ in a neighbourhood of the origin in $\mathbb{C}^{2 X}$ and in the variables $\left(z_{*}, z\right) \in \underset{\mathbf{x} \in X}{X} \mathcal{P}(\mathbf{x})$, with, for each $\mathbf{x} \in X, \mathcal{P}(\mathbf{x})$ an open neighbourhood of $\mathcal{B}(\mathbf{x})$. Then

$$
\begin{aligned}
\int_{D_{\mathbb{C}}} & \prod_{\mathbf{x} \in X}\left[\frac{d z_{*}(\mathbf{x}) \wedge d z(\mathbf{x})}{2 \pi i} e^{-z_{*}(\mathbf{x}) z(\mathbf{x})}\right] e^{f\left(\alpha_{*}, \beta ; z_{*}, z\right)} \\
= & \sum_{R \subset X} \prod_{\mathbf{x} \in R}\left(\int_{C(\mathbf{x})} \frac{d z_{*}(\mathbf{x}) \wedge d z(\mathbf{x})}{2 \pi i} e^{-z_{*}(\mathbf{x}) z(\mathbf{x})}\right) \\
& \left.\prod_{\mathbf{x} \in X \backslash R}\left(\int_{|z(\mathbf{x})| \leq r} \frac{d z(\mathbf{x})^{*} \wedge d z(\mathbf{x})}{2 \pi i} e^{-z_{*}(\mathbf{x}) z(\mathbf{x})}\right) e^{f\left(\alpha_{*}, \beta ; z_{*}, z\right)}\right|_{\substack{z_{*}(\mathbf{x})=z(\mathbf{x}) * \\
\text { for } \mathbf{x} \in X \backslash R}}
\end{aligned}
$$


(b) We have

$$
\operatorname{Re}\left(z_{*}(\mathbf{x}) z(\mathbf{x})\right) \geq \frac{1}{2}\left(r^{2}-|\rho(\mathbf{x})|^{2}\right)
$$

for all $\left(z_{*}(\mathbf{x}), z(\mathbf{x})\right) \in C(\mathbf{x})$. Furthermore the area of $C(\mathbf{x})$ is bounded by $4 \pi r|\rho|$. That is,

$$
\left|\int_{C(\mathbf{x})} \frac{d z_{*}(\mathbf{x}) \wedge d z(\mathbf{x})}{2 \pi i} f\left(z_{*}(\mathbf{x}), z(\mathbf{x})\right)\right| \leq 2 r|\rho| \sup _{C(\mathbf{x})}\left|f\left(z_{*}(\mathbf{x}), z(\mathbf{x})\right)\right|
$$

Proof (a) We apply Stokes' Theorem once for each point $\mathbf{x} \in X$ to the differential form

$$
\omega=\bigwedge_{\mathbf{x} \in X} \frac{d z_{*}(\mathbf{x}) \wedge d z(\mathbf{x})}{2 \pi i} \exp \left\{-\left\langle z_{*}, z\right\rangle+f\left(\alpha_{*}, \beta ; z_{*}, z\right)\right\}
$$

Since $\omega$ is a holomorphic $2|X|$ form in $\mathbb{C}^{2|X|}, d \omega=0$ and

$$
\int_{D} \omega=\sum_{R \subset X} \int_{M_{R}} \omega \quad \text { where } \quad M_{R}=\prod_{\mathbf{x} \notin R} D_{\mathbb{R}}(\mathbf{x}) \times \prod_{\mathbf{x} \in R} C(\mathbf{x})
$$

(b) Let $\left(z_{*}, z\right) \in C(\mathbf{x})$. We suppress the dependence on $\mathbf{x}$. There is a $0 \leq t \leq 1$ such that $\max \left\{\left|z_{*}\right|,|z|\right\}=r$ and $z_{*}=z^{*}-t \rho^{*}$. So

$$
\begin{aligned}
& z_{*} z=|z|^{2}-t \rho^{*} z \\
& z_{*} z=\left|z_{*}\right|^{2}+t \rho z^{*}-|t \rho|^{2}
\end{aligned}
$$

Adding and taking the real part,

$$
2 \operatorname{Re}\left(z_{*} z\right)=|z|^{2}+\left|z_{*}\right|^{2}-t^{2}|\rho|^{2} \geq r^{2}-|\rho|^{2}
$$

By construction, $C(\mathbf{x})$ is contained in the union of the two cylinders

$$
\begin{aligned}
& U=\left\{\left(r e^{-i \theta}-t \rho^{*}, r e^{i \theta}\right) \mid \theta \in[0,2 \pi], t \in[0,1]\right\} \\
& L=\left\{\left(r e^{i \theta}, r e^{-i \theta}+t \rho\right) \mid \theta \in[0,2 \pi], t \in[0,1]\right\}
\end{aligned}
$$

The upper cylinder contains the part of $C(\mathbf{x})$ with $|z(\mathbf{x})|=r$ and the lower cylinder contains the part with $z_{*}(\mathbf{x})=r$. We'll bound the integral over the upper cylinder. On $U$, we have $d z=i r e^{i \theta} d \theta$ and $d z_{*}=-i r e^{-i \theta} d \theta-\rho^{*} d t$, which gives

$$
d z_{*} \wedge d z=-i \rho^{*} r e^{i \theta} d t \wedge d \theta
$$

since $d \theta \wedge d \theta=0$. Hence

$$
\left|\int_{U} \frac{d z_{*} \wedge d z}{2 \pi i} f\left(z_{*}, z\right)\right| \leq \frac{r|\rho|}{2 \pi} \int_{0}^{2 \pi} d \theta \int_{0}^{1} d t\left|f\left(r e^{-i \theta}-t \rho^{*}, r e^{i \theta}\right)\right| \leq r|\rho| \sup _{U}\left|f\left(z_{*}, z\right)\right|
$$




\section{The Temporal Ultraviolet Limit}

\subsubsection{The Error}

We finish off this section by hinting at why the error introduced by the stationary phase approximation is extremely small. We consider the case $n=0$. The initial functional integral representation (1.8) may be written

$$
\begin{array}{r}
\operatorname{Tr} e^{-\frac{1}{k T}(H-\mu N)}=\lim _{\varepsilon \rightarrow 0} \int_{\tau \in \varepsilon \mathbb{Z} \cap\left(0, \frac{1}{k T}\right]} \prod_{\mathbf{x} \in X}\left\{\left[\prod_{\frac{d \alpha_{\tau}^{*}(\mathbf{x}) \wedge d \alpha_{\tau}(\mathbf{x})}{2 \pi \imath}} \chi\left(\left|\alpha_{\tau}(\mathbf{x})\right|<\mathrm{R}(\varepsilon)\right)\right]\right. \\
\left.e^{-\frac{1}{2}\left\langle\alpha_{\tau-\varepsilon}^{*}, \alpha_{\tau-\varepsilon}\right\rangle} I_{0}\left(\varepsilon ; \alpha_{\tau-\varepsilon}^{*}, \alpha_{\tau}\right) e^{-\frac{1}{2}\left\langle\alpha_{\tau}^{*}, \alpha_{\tau}\right\rangle}\right\}
\end{array}
$$

where

$$
I_{0}\left(\varepsilon ; \alpha^{*}, \beta\right)=e^{\left\langle\alpha^{*}, j(\varepsilon) \beta\right\rangle} e^{-\varepsilon\left\langle\alpha^{*} \beta, v \alpha^{*} \beta\right\rangle} \zeta_{\varepsilon}(\alpha, \beta)
$$

(a) We first discuss why inserting the "time derivative small field characteristic functions" $\zeta_{\varepsilon}(\alpha, \beta)$, with $\alpha=\alpha_{\tau-\varepsilon}$ and $\beta=\alpha_{\tau}$ for the various different values of $\tau$, (which are not present in the formal functional integral (1.1)) introduced only a very small error, which tends to zero quickly in the limit $\varepsilon \rightarrow 0$. The critical observation is that the quadratic part of the exponent of $e^{-\frac{1}{2}\left\langle\alpha^{*}, \alpha\right\rangle} I_{0}\left(\varepsilon ; \alpha^{*}, \beta\right) e^{-\frac{1}{2}\left\langle\beta^{*}, \beta\right\rangle}$ obeys

$$
\begin{aligned}
\operatorname{Re}\left\{-\frac{1}{2}\left\langle\alpha^{*}, \alpha\right\rangle+\left\langle\alpha^{*}, j(\varepsilon) \beta\right\rangle-\frac{1}{2}\left\langle\beta^{*}, \beta\right\rangle\right\} & \approx \operatorname{Re}\left\{-\frac{1}{2}\left\langle\alpha^{*}, \alpha\right\rangle+\left\langle\alpha^{*}, \beta\right\rangle-\frac{1}{2}\left\langle\beta^{*}, \beta\right\rangle\right\} \\
& =-\frac{1}{2}\|\alpha-\beta\|_{L^{2}}^{2}
\end{aligned}
$$

which generates a factor of order $e^{-\frac{1}{2} \mathrm{r}(\varepsilon)^{2}}$ when $(\alpha, \beta)$ is not in the support of $\zeta_{\varepsilon}(\alpha, \beta)$. This factor will be miniscule, because we shall choose $\mathrm{r}(\varepsilon)=\frac{1}{(\varepsilon \mathfrak{v})^{1 / 20}}$ where $\mathfrak{v}$ is a small positive constant (and $\frac{1}{20}$ is a randomly chosen small positive number).

(b) A similar mechanism generates small factors whenever the difference $\beta-\alpha$ (now think of this as $\alpha_{\tau}-\alpha_{\tau-2 \varepsilon}$ ) between the two arguments of

$$
I_{1}\left(\varepsilon ; \alpha^{*}, \beta\right)=\int d \mu_{\mathrm{R}(\varepsilon)}\left(\phi^{*}, \phi\right) I_{0}\left(\varepsilon ; \alpha^{*}, \phi\right) I_{0}\left(\varepsilon ; \phi^{*}, \beta\right)
$$

is larger than roughly $\mathrm{r}(2 \varepsilon)$. Consequently, we use the stationary phase approximation for this integral only when the "time derivative small field condition" $\|\alpha-\beta\|_{\infty} \leq \mathrm{r}(2 \varepsilon)$ is satisfied. The change of variables (1.21) expresses $I_{1}$ as

$$
\begin{aligned}
I_{1}\left(\varepsilon ; \alpha^{*}, \beta\right)=e^{\left\langle\alpha^{*}, j(2 \varepsilon) \beta\right\rangle}\left[\prod_{\mathbf{x} \in X} \int_{M(\mathbf{x})} \frac{d z_{*}(\mathbf{x}) \wedge d z(\mathbf{x})}{2 \pi \imath} e^{-z_{*}(\mathbf{x}) z(\mathbf{x})}\right] e^{\tilde{\mathcal{A}}\left(\alpha^{*}, \beta ; z_{*}, z\right)} \\
\zeta_{\varepsilon}(\alpha, j(\varepsilon) \beta+z) \zeta_{\varepsilon}\left(\left(j(\varepsilon) \alpha^{*}+z_{*}\right)^{*}, \beta\right)
\end{aligned}
$$

The characteristic function $\zeta_{\varepsilon}(\alpha, j(\varepsilon) \beta+z)$ limits the domain of integration to $z$ 's obeying

$$
\|z+j(\varepsilon) \beta-\alpha\|_{\infty}<\mathrm{r}(\varepsilon)
$$

Since $\|\alpha-\beta\|_{\infty} \leq \mathrm{r}(2 \varepsilon)=\frac{1}{2^{1 / 20}} \mathrm{r}(\varepsilon)$ and $\|j(\varepsilon) \beta-\beta\|_{\infty} \leq \operatorname{const} \varepsilon \mathrm{R}(\varepsilon) \ll \mathrm{r}(\varepsilon)$ (by our choice of $\mathrm{R}(\varepsilon)$ - see part (d), below), this condition is more or less equivalent to 
$\|z\|_{\infty}<\mathrm{r}(\varepsilon)$. Indeed, on the difference between the domain $\|z+j(\varepsilon) \beta-\alpha\|_{\infty}<\mathrm{r}(\varepsilon)$ and the domain $\|z\|_{\infty}<\mathrm{r}(\varepsilon)$, the integrand is extremely small, for reasons like those given in part (a), above. Similarly, the condition imposed by the second $\zeta_{\varepsilon}$ is roughly equivalent to $\left\|z_{*}\right\|_{\infty}<\mathrm{r}(\varepsilon)$. The two conditions $\|z\|_{\infty} \leq \mathrm{r}(\varepsilon)$ and $\left\|z_{*}\right\|_{\infty} \leq \mathrm{r}(\varepsilon)$ are built into the domains of integration $D(\mathbf{x})$ in (1.22).

(c) The "time derivative small field condition" $\|\alpha-\beta\|_{\infty} \leq \mathrm{r}(2 \varepsilon)=\frac{1}{2^{1 / 20}} \mathrm{r}(\varepsilon)$ is also used to ensure that $-z_{*}(\mathbf{x}) z(\mathbf{x})$ has an extremely large negative real part whenever $\left(z_{*}(\mathbf{x}), z(\mathbf{x})\right)$ lies on $C(\mathbf{x})$, the side of the Stokes' "cylinder". This may be seen from part (b) of Lemma 1.2, with $r=\mathrm{r}(\varepsilon)$ and $\rho=\left(\phi_{*}^{\text {crit }}\right)^{*}-\phi^{\text {crit }}=j(\varepsilon)[\alpha-\beta]$.

(d) Another mechanism, which is similar in spirit to, but different from, the supression of large time derivatives, arises from the $e^{-\varepsilon\left\langle\alpha^{*} \beta, v \alpha^{*} \beta\right\rangle}$ in (1.25). When $\alpha \approx \beta$ (i.e. when the time derivative is small), the exponent is roughly

$$
-\varepsilon\left\langle\alpha^{*} \alpha, v \alpha^{*} \alpha\right\rangle \leq-\varepsilon \mathfrak{v}_{1}\left\langle\alpha^{*} \alpha, \alpha^{*} \alpha\right\rangle=-\varepsilon \mathfrak{v}_{1} \sum_{\mathbf{x} \in X}|\alpha(\mathbf{x})|^{4}
$$

where $\mathfrak{v}_{1}$ is the smallest eigenvalue of the integral operator with kernel $v(\mathbf{x}, \mathbf{y})$. Recall that we have assumed that the integral operator with kernel $v(\mathbf{x}, \mathbf{y})$ is strictly positive. So if for some $\mathbf{x} \in X$, we have $|\alpha(\mathbf{x})| \geq \mathrm{R}(\varepsilon)$, then

$$
e^{-\varepsilon\left\langle\alpha^{*} \alpha, v \alpha^{*} \alpha\right\rangle} \leq e^{-\mathfrak{v}_{1} \varepsilon \mathrm{R}(\varepsilon)^{4}}
$$

The large field cutoff $\mathrm{R}(\varepsilon)$ is chosen so that this is, again, minuscule when $\varepsilon$ is small. For example, $\mathrm{R}(\varepsilon)=\frac{1}{(\varepsilon \mathfrak{v})^{3 / 10}}$, (with $\frac{3}{10}$ a randomly chosen number that is strictly bigger than, but close to $\frac{1}{4}$ ) does the job.

\subsection{Bounds on the Stationary Phase Approximation}

In this section, we outline the proof of some bounds on the $\mathcal{E}_{\delta}\left(\varepsilon ; \alpha^{*}, \beta\right)$ 's of (1.16). The bounds are expressed in terms of a family of norms on analytic functions of $\left\{\alpha^{*}(\mathbf{x}), \beta(\mathbf{x}) \mid \mathbf{x} \in X\right\}$.

An analytic function $f\left(\alpha^{*}, \beta\right)$ of $\alpha^{*}$ and $\beta$ may be expanded in a power series

$f\left(\alpha^{*}, \beta\right)=\sum_{k, \ell \geq 0} \sum_{\substack{\mathbf{x}_{1}, \cdots, \mathbf{x}_{k} \in X \\ \mathbf{y}_{1}, \cdots, \mathbf{y}_{\ell} \in X}} a\left(\mathbf{x}_{1}, \cdots, \mathbf{x}_{k} ; \mathbf{y}_{1}, \cdots, \mathbf{y}_{\ell}\right) \alpha\left(\mathbf{x}_{1}\right)^{*} \cdots \alpha\left(\mathbf{x}_{k}\right)^{*} \beta\left(\mathbf{y}_{1}\right) \cdots \beta\left(\mathbf{y}_{\ell}\right)$

(with the coefficients $a\left(\mathbf{x}_{1}, \cdots, \mathbf{x}_{k} ; \mathbf{y}_{1}, \cdots, \mathbf{y}_{\ell}\right)$ invariant under permutations of $\mathbf{x}_{1}$, $\cdots, \mathbf{x}_{k}$ and of $\left.\mathbf{y}_{1}, \cdots, \mathbf{y}_{\ell}\right)$. For the functions of interest, the "symmetric coefficient system" $a\left(\mathbf{x}_{1}, \cdots, \mathbf{x}_{k} ; \mathbf{y}_{1}, \cdots, \mathbf{y}_{\ell}\right)$ will be translation invariant (recall that $X$ is the finite discrete torus $\mathbb{Z}^{3} /(L \mathbb{Z})^{3}$, for some large $L \in \mathbb{N}$ ) but otherwise exponentially decaying (uniformly in $L$ ). We tailor our norms to these two characteristics by defining the norm

$$
\left\|f\left(\alpha^{*}, \beta\right)\right\|_{\delta}=\sum_{k, \ell \geq 0} \max _{\mathbf{x} \in X} \max _{1 \leq i \leq k+\ell} \sum_{\substack{(\overrightarrow{\mathbf{x}}, \vec{y}) \in X^{k} \times X^{\ell} \\(\mathbf{x}, \vec{y})_{i}=\mathbf{x}}} w_{\delta}(\overrightarrow{\mathbf{x}} ; \overrightarrow{\mathbf{y}})|a(\overrightarrow{\mathbf{x}} ; \overrightarrow{\mathbf{y}})|
$$




\section{The Temporal Ultraviolet Limit}

with the "weight system"

$$
w_{\delta}(\overrightarrow{\mathbf{x}} ; \overrightarrow{\mathbf{y}})=\kappa(\delta)^{k+\ell} e^{\operatorname{md}(\overrightarrow{\mathbf{x}}, \overrightarrow{\mathbf{y}})} \quad \text { for }(\overrightarrow{\mathbf{x}}, \overrightarrow{\mathbf{y}}) \in X^{k} \times X^{\ell}
$$

where $\tau(\overrightarrow{\mathbf{x}}, \overrightarrow{\mathbf{y}})$ is the minimal length of a tree whose set of vertices contains the set $\left\{\mathbf{x}_{1}, \cdots, \mathbf{x}_{k}, \mathbf{y}_{1}, \cdots, \mathbf{y}_{\ell}\right\}$. We refer to (1.27) as the weight system with mass $m$ that associates the constant weight factor $\kappa(\delta)$ to the fields $\alpha^{*}$ and $\beta$. During the course of the proof, we will use other similar norms, with different weights. Roughly speaking, for $\left\|f\left(\alpha^{*}, \beta\right)\right\|_{\delta}$ to be finite, each coefficient $a\left(\mathbf{x}_{1}, \cdots, \mathbf{x}_{k} ; \mathbf{y}_{1}, \cdots, \mathbf{y}_{\ell}\right)$

- must decay a bit better than exponentially with rate $\mathrm{m}$ when one argument is held fixed and at least one other argument is moved far away and

- must be of size smaller than $\frac{1}{\kappa(\delta)^{k+\ell}}$. (The weight $\kappa(\delta)$ will be chosen shortly.)

If $\left\|f\left(\alpha^{*}, \beta\right)\right\|_{\delta}$ is finite, then $f\left(\alpha^{*}, \beta\right)$ is analytic, and bounded by $|X|\left\|f\left(\alpha^{*}, \beta\right)\right\|_{\delta}$, on the domain $\left\{\left(\alpha^{*}, \beta\right) \in \mathbb{C}^{2|X|}|| \alpha(\mathbf{x})|,| \beta(\mathbf{x}) \mid<\kappa(\delta)\right.$ for all $\left.\mathbf{x} \in X\right\}$.

The decay properties of $\mathcal{E}_{n}$ 's arise from the decay properties of the operators $j(\tau)=$ $e^{-\tau(\mathrm{h}-\mu)}$ and $v$ in the initial $I_{0}$ of Theorem 1.1. In general, we capture the decay properties of any operator $\mathcal{A}$ on $L^{2}(X)=\mathbb{C}^{X}$, with $\operatorname{kernel} \mathcal{A}(\mathbf{x}, \mathbf{y})$ (i.e. that maps $\varphi(\mathbf{x}) \in L^{2}(X)$ to $\left.(\mathcal{A} \varphi)(\mathbf{x})=\sum_{\mathbf{y} \in X} \mathcal{A}(\mathbf{x}, \mathbf{y}) \varphi(\mathbf{y}) \in L^{2}(X)\right)$, by using the weighted $L^{1}-L^{\infty}$ operator norm

$$
\|\| \mathcal{A} \|=\max \left\{\sup _{\mathbf{x} \in X} \sum_{\mathbf{y} \in X} e^{\operatorname{md}(\mathbf{x}, \mathbf{y})}|\mathcal{A}(\mathbf{x}, \mathbf{y})|, \sup _{\mathbf{y} \in X} \sum_{\mathbf{x} \in X} e^{\operatorname{md}(\mathbf{x}, \mathbf{y})}|\mathcal{A}(\mathbf{x}, \mathbf{y})|\right\}
$$

where $d(\mathbf{x}, \mathbf{y})$ is the metric on $X=\mathbb{Z}^{3} / L \mathbb{Z}^{3}$. Some useful properties of this norm are given in

Lemma 1.3 (a) For any two operators $\mathcal{A}, \mathcal{B}: L^{2}(X) \rightarrow L^{2}(X)$

$$
\|\mathcal{A B}\||\leq\|\mathcal{A}|\|\mid\| \mathcal{B} \|
$$

(b) For any operator $\mathcal{A}: L^{2}(X) \rightarrow L^{2}(X)$ and any complex number $\alpha$

$$
\left\|e^{\alpha \mathcal{A}}\right\| \leq e^{|\alpha|\|\mathcal{A} \mid\|} \quad\left\||| e^{\alpha \mathcal{A}}-1\left|\left\|\leq|\alpha|\left|\|\mathcal{A} \mid\| e^{|\alpha|\|\mathcal{A}\| \mid}\right.\right.\right.\right.
$$

Proof (a) By the triangle inequality, for each $\mathbf{x} \in X$,

$$
\begin{aligned}
\sum_{\mathbf{y} \in X} e^{\operatorname{md}(\mathbf{x}, \mathbf{y})}|(\mathcal{A B})(\mathbf{x}, \mathbf{y})| & \leq \sum_{\mathbf{y}, \mathbf{z} \in X} e^{\operatorname{md}(\mathbf{x}, \mathbf{z})}|\mathcal{A}(\mathbf{x}, \mathbf{z})| e^{\operatorname{md}(\mathbf{z}, \mathbf{y})}|\mathcal{B}(\mathbf{z}, \mathbf{y})| \\
& \leq \sum_{\mathbf{z} \in X} e^{\operatorname{md}(\mathbf{x}, \mathbf{z})}|\mathcal{A}(\mathbf{x}, \mathbf{z})|\|\mathcal{B}\| \mid \\
& \leq\|\mathcal{A}\||\|\mathcal{B}\||
\end{aligned}
$$

The other bound, in which one sums over $\mathbf{x}$ rather than $\mathbf{y}$, is similar. 
(b) By part (a),

$$
\left\|e^{\alpha \mathcal{A}}\right\| \leq \sum_{n=0}^{\infty} \frac{1}{n !}\left\|\alpha^{n} \mathcal{A}^{n}\right\| \leq \sum_{n=0}^{\infty} \frac{1}{n !}|\alpha|^{n}\|\mid \mathcal{A}\|^{n}=e^{|\alpha|\|\mathcal{A}\| \|}
$$

and

$$
\left.\left\|e^{\alpha \mathcal{A}}-\mathbb{1}\left|\left\|\leq \sum_{n=1}^{\infty} \frac{1}{n !}\right\|\right| \alpha^{n} \mathcal{A}^{n}\right\|\left|\leq \sum_{n=1}^{\infty} \frac{1}{n !}\right| \alpha\right|^{n}\left|\|\mathcal{A}\|^{n} \leq\right| \alpha \mid\|\mathcal{A}\| e^{|\alpha|\|\mathcal{A}\|}
$$

Corollary 1.4 Let $\tau \geq 0$.

$$
\left\|\left|\|j(\tau)\| \leq e^{\tau(\|\mathrm{h}\| \mid+\mu)} \quad\|j(\tau)-\mathbb{1}\|\right| \leq \tau\left(\left|\|\mathrm{h}|\|+| \mu \mid) e^{\tau(\|\mathrm{h}\|+|\mu|)}\right.\right.\right.
$$

Proof Write $j(\tau)=e^{\tau \mu} e^{-\tau \mathrm{h}}$ and $j(\tau)-\mathbb{1}=e^{\tau \mu}\left(e^{-\tau \mathrm{h}}-\mathbb{1}\right)+e^{\tau \mu}-\mathbb{1}$. By the previous Lemma

$$
\|\| j(\tau)\left\|\left|=e^{\tau \mu}\left\|e^{-\tau \mathrm{h}}\right\|\right| \leq e^{\tau \mu} e^{\tau\|\mathrm{h}\|}\right.
$$

and

$$
|\|j(\tau)-11\|| \leq e^{\tau \mu}\left\|e^{-\tau \mathrm{h}}-\mathbb{1}\right\|\left|+\left\|e^{\tau \mu}-11\right\| \leq \tau\right|\|\mathrm{h}\| e^{\tau \mu} e^{\tau\|\mathrm{h}\|}+\left|e^{\tau \mu}-1\right|
$$

The quantities relevant for the estimates of $\mathcal{E}_{\delta}\left(\varepsilon ; \alpha^{*}, \beta\right)$, in addition to the radii $\mathrm{r}(\delta)$, of the domain of integration, and $\kappa(\delta)$, of the domain of analyticity, are the norm $\|v\| \|$ of the interaction, the decay rate $\mathrm{m}$, a constant $K_{j}$ such

$$
\|j(\tau)\| \leq e^{K_{j} \tau} \quad \text { and } \quad\|j(\tau)-\mathbb{1}\| \leq K_{j} \tau e^{K_{j} \tau} \quad \text { for } \tau \geq 0
$$

(see Corollary 1.4) and a constant $0<\Theta \leq 1$ that bounds the range of $\theta$ 's (see (1.13)) for which the constructions work. In (Balaban et al., 2010b, Hypothesis 1.1) we give a set of hypotheses on these constants. (For the full temporal ultraviolet limit, not just the stationary phase approximation, see (Balaban et al., 2010c, Appendix F).) For the purposes of these lectures, I'll just make one reasonably specific choice. I'll allow any $K_{j}, \mathrm{~m}>0$ and view them just as fixed constants. Then I'll pick sufficiently small (depending on $K_{j}$ and $\mathrm{m}$ ) $0<\mathfrak{v}, \Theta \leq 1$ and allow any interaction $v$ with $\|v\| \|<\mathfrak{v}$. Then I'll set

$$
\mathrm{r}(\delta)=\frac{1}{(\delta \mathfrak{v})^{\frac{1}{20}}} \quad \kappa(\delta)=\frac{1}{(\delta \mathfrak{v})^{\frac{3}{10}}}
$$

Think of the exponents $\frac{1}{20}$ and $\frac{3}{10}$ as being just a tiny bit bigger than 0 and $\frac{1}{4}$, respectively.

I will outline the proof of 


\section{The Temporal Ultraviolet Limit}

Theorem 1.5 Under the above hypotheses, there is a constant $K_{E}$ such that

$$
\left\|\mathcal{E}_{\delta}\left(\varepsilon ; \alpha^{*}, \beta\right)\right\|_{\delta} \leq K_{E} \delta^{2}\|v\|^{2} \mathrm{r}(\delta)^{2} \kappa(\delta)^{6}
$$

for all $0 \leq \varepsilon \leq \delta \leq \Theta$ for which $\frac{\delta}{\varepsilon}$ is a power of 2 . The function $\mathcal{E}_{\delta}\left(\varepsilon ; \alpha^{*}, \beta\right)$ has degree at least two both ${ }^{4}$ in $\alpha^{*}$ and $\beta$.

In (Balaban et al., 2010b, Theorem 1.4), we also prove

Theorem 1.6 The limit

$$
\mathcal{E}_{\theta}\left(\alpha^{*}, \beta\right)=\lim _{m \rightarrow \infty} \mathcal{E}_{\theta}\left(2^{-m} \theta ; \alpha^{*}, \beta\right)
$$

exists uniformly in $0 \leq \theta \leq \Theta$. It fulfills the estimate

$$
\left\|\mathcal{E}_{\theta}\left(\alpha^{*}, \beta\right)\right\|_{\theta} \leq K_{E} \theta^{2}\|v\|^{2} \mathrm{r}(\theta)^{2} \kappa(\theta)^{6}
$$

and has degree at least two in both $\alpha^{*}$ and $\beta$.

The proof of Theorem 1.6 uses the same techniques as the proof of Theorem 1.5. So I won't discuss the former at all.

Remark 1.7 Theorem 1.5 implies that $\left\|\mathcal{E}_{\delta}\left(\varepsilon ; \alpha^{*}, \beta\right)\right\|_{\delta} \leq K_{E}\left(\frac{\|v\|}{\mathfrak{v}}\right)^{2}$ for all $0 \leq \varepsilon \leq$ $\delta \leq \Theta$. In particular $\mathcal{E}_{\delta}\left(\varepsilon ; \alpha^{*}, \beta\right)$ is analytic and bounded pointwise by $K_{E}|X|\left(\frac{\|v\| \|}{\mathfrak{v}}\right)^{2}$ on $\left\{\left(\alpha^{*}, \beta\right) \in \mathbb{C}^{2|X|}|| \alpha(\mathbf{x})|,| \beta(\mathbf{x}) \mid<(\delta \mathfrak{v})^{-\frac{3}{10}}\right.$ for all $\left.\mathbf{x} \in X\right\}$. The coefficients in its power series expansion decay exponentially at rate at least $\mathrm{m}$.

We formulate the recursion relation (1.16) that defines $\mathcal{E}_{\varepsilon_{n}}\left(\varepsilon ; \alpha^{*}, \beta\right)$ more abstractly.

Definition 1.8 Let $0 \leq \varepsilon \leq \delta$. For an action $\mathcal{E}\left(\alpha^{*}, \beta\right)$ we set

$$
\mathfrak{R}_{\delta, \varepsilon}[\mathcal{E}]\left(\alpha^{*}, \beta\right)=\mathcal{E}\left(\alpha^{*}, j(\delta) \beta\right)+\mathcal{E}\left(j(\delta) \alpha^{*}, \beta\right)+\log \frac{\int d \mu_{\mathrm{r}(\delta)}\left(z^{*}, z\right) e^{\partial \mathcal{A}_{\delta, \varepsilon}\left(\mathcal{E} ; \alpha^{*}, \beta ; z^{*}, z\right)}}{\int d \mu_{\mathrm{r}(\delta)}\left(z^{*}, z\right)}
$$

whenever the logarithm is defined. Here

$$
\begin{aligned}
\partial \mathcal{A}_{\delta, \varepsilon}\left(\mathcal{E} ; \alpha^{*}, \beta ; z_{*}, z\right)= & {\left[\mathcal{V}_{\delta}\left(\varepsilon ; \alpha^{*}, j(\delta) \beta+z\right)-\mathcal{V}_{\delta}\left(\varepsilon ; \alpha^{*}, j(\delta) \beta\right)\right] } \\
& +\left[\mathcal{V}_{\delta}\left(\varepsilon ; j(\delta) \alpha^{*}+z_{*}, \beta\right)-\mathcal{V}_{\delta}\left(\varepsilon ; j(\delta) \alpha^{*}, \beta\right)\right] \\
& +\left[\mathcal{E}\left(\alpha^{*}, j(\delta) \beta+z\right)-\mathcal{E}\left(\alpha^{*}, j(\delta) \beta\right)\right] \\
& +\left[\mathcal{E}\left(j(\delta) \alpha^{*}+z_{*}, \beta\right)-\mathcal{E}\left(j(\delta) \alpha^{*}, \beta\right)\right]
\end{aligned}
$$

\footnotetext{
${ }^{4}$ By this we mean that every monomial appearing in its power series expansion contains a factor of the form $\alpha^{*}\left(\mathbf{x}_{1}\right) \alpha^{*}\left(\mathbf{x}_{2}\right) \beta\left(\mathbf{x}_{3}\right) \beta\left(\mathbf{x}_{4}\right)$.
} 
The recursion relation (1.16) is equivalent to

$$
\begin{aligned}
\mathcal{E}_{\varepsilon}\left(\varepsilon ; \alpha^{*}, \beta\right) & =0 \\
\mathcal{E}_{\varepsilon_{n+1}}\left(\varepsilon ; \alpha^{*}, \beta\right) & =\mathfrak{R}_{\varepsilon_{n}, \varepsilon}\left[\mathcal{E}_{\varepsilon_{n}}\left(\varepsilon ; \alpha^{*}, \beta\right)\right]
\end{aligned}
$$

To prove Theorem 1.5 , we perform induction on $n$ to successively bound $\mathcal{E}_{\varepsilon_{n}}(\varepsilon ; \cdot)$ for $n=0, \cdots, \log _{2} \frac{\Theta}{\varepsilon}$. The heart of the induction step is given in Proposition 1.11. Proposition 1.11, in turn, is an application of a corollary to (Balaban et al., 2010a, Theorem 3.4), which, specialized to the current setting, says

Theorem 1.9 Let $\kappa>0$ and denote by $\|\cdot\|_{\mathrm{fl}}$ the norm ${ }^{5}$ with weight system of mass $\mathrm{m}$ that assigns the weight $\kappa>0$ to the fields $\alpha^{*}$ and $\beta$ and the weight $4 \mathrm{r}(\delta)$ to the fields $z_{*}$ and $z$. If $f\left(\alpha^{*}, \beta ; z_{*}, z\right)$ is an analytic function on a neighbourhood of the origin in $\mathbb{C}^{4|X|}$ that obeys $\|f\|_{\mathrm{f}}<\frac{1}{16}$, then there is an analytic function $g\left(\alpha^{*}, \beta\right)$ such that

$$
\frac{\int e^{f\left(\alpha^{*}, \beta ; z^{*}, z\right)} d \mu_{\mathrm{r}(\delta)}\left(z^{*}, z\right)}{\int e^{f\left(0,0 ; z^{*}, z\right)} d \mu_{\mathrm{r}(\delta)}\left(z^{*}, z\right)}=e^{g\left(\alpha^{*}, \beta\right)}
$$

and

$$
\|g\|_{\mathrm{fl}} \leq \frac{\|f\|_{\mathrm{fl}}}{1-16\|f\|_{\mathrm{fl}}}
$$

I'll give an outline of the proof of this theorem in $\S 1.5$. See Theorem 1.29. The corollary that we shall use is (Balaban et al., 2010a, Corollary 3.5), which, again specialized to the current setting, says

Corollary 1.10 Let $f\left(\alpha^{*}, \beta ; z_{*}, z\right)$ be an analytic function on a neighbourhood of the origin in $\mathbb{C}^{4|X|}$ that obeys $\|f\|_{\mathrm{fl}}<\frac{1}{32}$. Define, for each complex number $\zeta$ with $|\zeta|\|f\|_{\mathrm{f}}<\frac{1}{16}$, the function $G(\zeta)=G\left(\zeta ; \alpha^{*}, \beta\right)$ by the condition

$$
\frac{\int e^{\zeta f\left(\alpha^{*}, \beta ; z^{*}, z\right)} d \mu_{\mathrm{r}(\delta)}\left(z^{*}, z\right)}{\int e^{\zeta f\left(0,0 ; z^{*}, z\right)} d \mu_{\mathrm{r}(\delta)}\left(z^{*}, z\right)}=e^{G\left(\zeta ; \alpha^{*}, \beta\right)}
$$

as in Theorem 1.9. Then $G(\zeta)$ is a (Banach space valued) analytic function of $\zeta$ and, for each $n \in \mathbb{N}$, the $g\left(\alpha^{*}, \beta\right)=G\left(1 ; \alpha^{*}, \beta\right)$ of Theorem 1.9 obeys

$$
\left\|g-\frac{d G}{d \zeta}(0)-\cdots-\frac{1}{n !} \frac{d^{n} G}{d \zeta^{n}}(0)\right\|_{\mathrm{fl}} \leq\left(\frac{\|f\|_{\mathrm{fl}}}{\frac{1}{20}-\|f\|_{\mathrm{fl}}}\right)^{n+1}
$$

We have $G(0)=0$ and

$$
\frac{d G}{d \zeta}(0)=\int\left[f\left(\alpha^{*}, \beta ; z^{*}, z\right)-f\left(0,0 ; z^{*}, z\right)\right] d \mu_{\mathrm{r}(\delta)}\left(z^{*}, z\right)
$$

If the symmetric coefficient system $a\left(\overrightarrow{\mathbf{x}}_{*}, \overrightarrow{\mathbf{x}} ; \overrightarrow{\mathbf{y}}_{*}, \overrightarrow{\mathbf{y}}\right)$ of $f$ obeys $a\left(\overrightarrow{\mathbf{x}}_{*}, \overrightarrow{\mathbf{x}} ; \overrightarrow{\mathbf{y}}_{*}, \overrightarrow{\mathbf{y}}\right)=0$ whenever $\overrightarrow{\mathbf{y}}=\overrightarrow{\mathbf{y}}_{*}$, then $\frac{d G}{d \zeta}(0)=0$.

\footnotetext{
${ }^{5}$ The "fl" in $\|\cdot\|_{\mathrm{fl}}$ stands for fluctutation. This norm is defined just as in (1.26), except that there are four fields, $\alpha^{*}, \beta, z_{*}$ and $z$, instead of two, and the $\kappa(\delta)^{k+\ell}$ of $(1.27)$ is replaced by $\kappa^{k+\ell}(4 \mathrm{r}(\delta))^{n_{*}+n}$, where $k$ is the number of $\alpha^{*}$ fields, $\ell$ is the number of $\beta$ fields, $n_{*}$ is the number of $z_{*}$ fields and $n$ is the number of $z$ fields.
} 


\section{The Temporal Ultraviolet Limit}

Proof The proof of the bound in this corollary is a short, straight-forward application of the Cauchy integral formula. For the details, see (Balaban et al., 2010a, Corollary 3.5).

The left hand side is 1 when $\alpha^{*}=\beta=0$, so $G(0)=0$. To show that $\frac{d G}{d \zeta}(0)=$ 0 , under the specified conditions on the coefficient system, expand $f\left(\alpha^{*}, \beta ; z^{*}, z\right)$ in powers of the fields $\alpha^{*}, \beta, z^{*}$ and $z$. This expresses $\int f\left(\alpha^{*}, \beta ; z^{*}, z\right) d \mu_{\mathrm{r}(\delta)}\left(z^{*}, z\right)$ as a sum of terms, with each term being some coefficient (depending on $\alpha^{*}$ and $\beta$ ) times

$$
\int \prod_{\mathbf{x} \in X}\left\{\left[z(\mathbf{x})^{*}\right]^{n_{\mathbf{x}}} z(\mathbf{x})^{m_{\mathbf{x}}}\right\} d \mu_{\mathrm{r}(\delta)}\left(z^{*}, z\right)
$$

Switching to polar coordinates, $z(\mathbf{x})=\rho(\mathbf{x}) e^{i \theta(\mathbf{x})}$,

$$
\begin{aligned}
\int \prod_{\mathbf{x} \in X}\left\{\left[z(\mathbf{x})^{*}\right]^{n_{\mathbf{x}}}\right. & \left.z(\mathbf{x})^{m_{\mathbf{x}}}\right\} d \mu_{\mathrm{r}(\delta)}\left(z^{*}, z\right) \\
& =\prod_{\mathbf{x} \in X} \frac{1}{\pi} \int_{0}^{r(\delta)} d \rho(\mathbf{x}) \int_{0}^{2 \pi} d \theta(\mathbf{x}) e^{-\rho(\mathbf{x})^{2}} \rho(\mathbf{x})^{n_{\mathbf{x}}+m_{\mathbf{x}}+1} e^{i\left(m_{\mathbf{x}}-n_{\mathbf{x}}\right) \theta(\mathbf{x})}
\end{aligned}
$$

Unless $m_{\mathbf{x}}=n_{\mathbf{x}}$ for every $\mathbf{x} \in X$, the right hand side is zero because of the $\theta(\mathbf{x})$ integrals. When $m_{\mathbf{x}}=n_{\mathbf{x}}$ for every $\mathbf{x} \in X$, the coefficient multiplying this integral is zero because of the hypothesis on the symmetric coefficient system. Hence

$$
\int f\left(\alpha^{*}, \beta ; z^{*}, z\right) d \mu_{\mathrm{r}(\delta)}\left(z^{*}, z\right)=\int f\left(0,0 ; z^{*}, z\right) d \mu_{\mathrm{r}(\delta)}\left(z^{*}, z\right)=0
$$

For the induction step, we use

Proposition 1.11 For all $0 \leq \varepsilon \leq \delta \leq \Theta / 2$, with $\delta$ an integer multiple of $\varepsilon$, the following holds:

Let $\mathcal{E}\left(\alpha^{*}, \beta\right)$ be an analytic function which has degree at least two both in $\alpha^{*}$ and in $\beta$ and which obeys $\left\|\mathcal{E}\left(\alpha^{*}, \beta\right)\right\|_{\delta} \leq 4 e^{5 \delta K_{j}} \delta\|v\| \mid \mathrm{r}(\delta) \kappa(2 \delta)^{3}$. Then $\mathfrak{R}_{\delta, \varepsilon}[\mathcal{E}]\left(\alpha^{*}, \beta\right)$ is well defined, has degree at least two both in $\alpha^{*}$ and in $\beta$, and satisfies the estimate

$$
\left\|\Re_{\delta, \varepsilon}[\mathcal{E}]\right\|_{2 \delta} \leq 2^{20} e^{10 \delta K_{j}} \delta^{2}\|v\|^{2} \mathrm{r}(\delta)^{2} \kappa(2 \delta)^{6}+2 e^{2 \delta K_{j}}\left(\frac{\kappa(2 \delta)}{\kappa(\delta)}\right)^{4}\|\mathcal{E}\|_{\delta}
$$

Proof Observe that the functions

$$
\mathcal{V}_{\delta}\left(\varepsilon ; \alpha^{*}, j(\delta) \beta+z\right)-\mathcal{V}_{\delta}\left(\varepsilon ; \alpha^{*}, j(\delta) \beta\right) \quad \text { and } \quad \mathcal{E}\left(\alpha^{*}, j(\delta) \beta+z\right)-\mathcal{E}\left(\alpha^{*}, j(\delta) \beta\right)
$$

both have degree at least two in $\alpha^{*}$, degree at least one in $z$ and do not depend on $z_{*}$. Similarly, $\mathcal{V}_{\delta}\left(\varepsilon ; j(\delta) \alpha^{*}+z_{*}, \beta\right)-\mathcal{V}_{\delta}\left(\varepsilon ; j(\delta) \alpha^{*}, \beta\right)$ and $\mathcal{E}\left(j(\delta) \alpha^{*}+z_{*}, \beta\right)-\mathcal{E}\left(j(\delta) \alpha^{*}, \beta\right)$ have degree at least two in $\beta$, degree at least one in $z_{*}$ and do not depend on $z$. Since 
the integral of any monomial against $d \mu_{\mathrm{r}(\delta)}\left(z^{*}, z\right)$ is zero unless there are the same number of $z$ 's and $z^{*}$ 's (see (1.34)),

$$
\int d \mu_{\mathrm{r}(\delta)}\left(z^{*}, z\right) \partial \mathcal{A}_{\delta, \varepsilon}\left(\mathcal{E} ; \alpha^{*}, \beta ; z^{*}, z\right)=0
$$

and $\log \frac{\int d \mu_{\mathrm{r}(\delta)}\left(z^{*}, z\right) e^{\partial \mathcal{A}_{\delta, \varepsilon}\left(\mathcal{E} ; \alpha^{*}, \beta ; z^{*}, z\right)}}{\int d \mu_{\mathrm{r}(\delta)}\left(z^{*}, z\right)}$ has degree at least two both in $\alpha^{*}$ and in $\beta$. This implies that $\Re_{\delta, \varepsilon}[\mathcal{E}]\left(\alpha^{*}, \beta\right)$ has degree at least two both in $\alpha^{*}$ and in $\beta$.

We apply Corollary 1.10, with $\kappa=\kappa(2 \delta)$. Clearly, $\left\|f\left(\alpha^{*}, \beta\right)\right\|_{2 \delta}=\left\|f\left(\alpha^{*}, \beta\right)\right\|_{\mathrm{ff}}$ for functions that are independent of the fluctuation fields $z_{*}, z$. To apply the Corollary, we need to bound $\left\|\partial \mathcal{A}_{\delta, \varepsilon}\left(\mathcal{E} ; \alpha^{*}, \beta ; z_{*}, z\right)\right\|_{\mathrm{fl}}$.

We'll first bound

$$
\begin{aligned}
\mathcal{V}_{\delta}\left(\varepsilon ; \alpha^{*}, j(\delta) \beta+z\right) & -\mathcal{V}_{\delta}\left(\varepsilon ; \alpha^{*}, j(\delta) \beta\right) \\
= & \varepsilon \sum_{\tau \in \varepsilon \mathbb{Z} \cap[0, \delta)}\left[\left\langle\gamma_{* \tau} g_{\tau+\varepsilon}, v \gamma_{* \tau} g_{\tau+\varepsilon}\right\rangle-\left\langle\gamma_{* \tau} \hat{g}_{\tau+\varepsilon}, v \gamma_{* \tau} \hat{g}_{\tau+\varepsilon}\right\rangle\right]
\end{aligned}
$$

with

$$
\gamma_{* \tau}=j(\tau) \alpha^{*} \quad g_{\tau}=j(2 \delta-\tau) \beta \quad \hat{g}_{\tau}=j(\delta-\tau)(j(\delta) \beta+z)=j(2 \delta-\tau) \beta+j(\delta-\tau) z
$$

Expand out $\hat{g}_{\tau}$ as a sum of two terms, as in the last equation, expressing the summand $\left\langle\gamma_{* \tau} g_{\tau+\varepsilon}, v \gamma_{* \tau} g_{\tau+\varepsilon}\right\rangle-\left\langle\gamma_{* \tau} \hat{g}_{\tau+\varepsilon}, v \gamma_{* \tau} \hat{g}_{\tau+\varepsilon}\right\rangle$ itself as a sum of three terms, each of which is (except for a minus sign) of the form

$$
\begin{aligned}
& \left\langle\left(\Gamma_{1} \gamma_{1}\right)\left(\Gamma_{2} \gamma_{2}\right), v\left(\Gamma_{3} \gamma_{3}\right)\left(\Gamma_{4} \gamma_{4}\right)\right\rangle \\
& =\sum_{\substack{\mathbf{x}_{1}, \mathbf{x}_{2}, \mathbf{x}_{3}, \mathbf{x}_{4} \in X \\
\mathbf{y}_{1}, \mathbf{y}_{2} \in X}}\left[\prod_{\ell=1,2} \Gamma_{\ell}\left(\mathbf{y}_{1}, \mathbf{x}_{\ell}\right) \gamma_{\ell}\left(\mathbf{x}_{\ell}\right)\right] v\left(\mathbf{y}_{1}, \mathbf{y}_{2}\right)\left[\prod_{\ell=3,4} \Gamma_{\ell}\left(\mathbf{y}_{2}, \mathbf{x}_{\ell}\right) \gamma_{\ell}\left(\mathbf{x}_{\ell}\right)\right]
\end{aligned}
$$

with

$\Gamma_{1}=\Gamma_{2}=j(\tau-\varepsilon) \quad \gamma_{1}=\gamma_{3}=\alpha^{*} \quad\left(\Gamma_{3}, \gamma_{3}\right),\left(\Gamma_{4}, \gamma_{4}\right) \in\{(j(2 \delta-\tau), \beta),(j(\delta-\tau), z)\}$ and with at least one of $\left(\Gamma_{3}, \gamma_{3}\right),\left(\Gamma_{4}, \gamma_{4}\right)$ being $(j(\delta-\tau), z)$. In general

$$
\begin{aligned}
& \sum_{\substack{\mathbf{x}_{2}, \mathbf{x}_{3}, \mathbf{x}_{4} \in X \\
\mathbf{y}_{1}, \mathbf{y}_{2} \in X}} e^{\mathrm{m} \tau\left(\mathbf{x}_{1}, \mathbf{x}_{2}, \mathbf{x}_{3}, \mathbf{x}_{4}\right)}\left|\left[\prod_{\ell=1,2} \Gamma_{\ell}\left(\mathbf{y}_{1}, \mathbf{x}_{\ell}\right) \kappa_{\ell}\right] v\left(\mathbf{y}_{1}, \mathbf{y}_{2}\right)\left[\prod_{\ell=3,4} \Gamma_{\ell}\left(\mathbf{y}_{2}, \mathbf{x}_{\ell}\right) \kappa_{\ell}\right]\right| \\
& \leq \sum_{\substack{\mathbf{x}_{2}, \mathbf{x}_{3}, \mathbf{x}_{4} \\
\mathbf{y}_{1}, \mathbf{y}_{2}}}\left|\left[\prod_{\ell=1,2} e^{\mathrm{m} d\left(\mathbf{x}_{\ell}, \mathbf{y}_{1}\right)} \Gamma_{\ell}\left(\mathbf{y}_{1}, \mathbf{x}_{\ell}\right) \kappa_{\ell}\right] v\left(\mathbf{y}_{1}, \mathbf{y}_{2}\right) e^{\mathrm{m} d\left(\mathbf{y}_{1}, \mathbf{y}_{2}\right)}\left[\prod_{\ell=3,4} e^{\mathrm{m} d\left(\mathbf{x}_{\ell}, \mathbf{y}_{2}\right)} \Gamma_{\ell}\left(\mathbf{y}_{2}, \mathbf{x}_{\ell}\right) \kappa_{\ell}\right]\right| \\
& \leq \prod_{\ell=1}^{4} \kappa_{\ell} \sum_{\substack{\mathbf{x}_{2} \in X \\
\mathbf{y}_{1}, \mathbf{y}_{2} \in X}}\left[\prod_{\ell=1,2} e^{\mathrm{m} d\left(\mathbf{x}_{\ell}, \mathbf{y}_{1}\right)}\left|\Gamma_{\ell}\left(\mathbf{y}_{1}, \mathbf{x}_{\ell}\right)\right|\right]\left|v\left(\mathbf{y}_{1}, \mathbf{y}_{2}\right)\right| e^{\mathrm{m} d\left(\mathbf{y}_{1}, \mathbf{y}_{2}\right)}\left\|\left|\Gamma_{3}\|\mid\| \Gamma_{4} \|\right.\right. \\
& \leq \prod_{\ell=1}^{4} \kappa_{\ell} \sum_{\mathbf{x}_{2}, \mathbf{y}_{1} \in X}\left[\prod_{\ell=1,2} e^{\mathrm{m} d\left(\mathbf{x}_{\ell}, \mathbf{y}_{1}\right)}\left|\Gamma_{\ell}\left(\mathbf{y}_{1}, \mathbf{x}_{\ell}\right)\right|\right]\|v\|\|\| \Gamma_{3}\|\mid\| \Gamma_{4}\|\|
\end{aligned}
$$




\section{The Temporal Ultraviolet Limit}

$$
\leq\|v\|\left\|\prod_{\ell=1}^{4} \kappa_{\ell}\right\| \Gamma_{\ell} \|
$$

To get from the first line to the second line, we used that the set of vertices of the tree in the figure below contains $\mathbf{x}_{1}, \mathbf{x}_{2}, \mathbf{x}_{3}$ and $\mathbf{x}_{4}$ so that

$$
\tau\left(\mathbf{x}_{1}, \mathbf{x}_{2}, \mathbf{x}_{3}, \mathbf{x}_{4}\right) \leq d\left(\mathbf{x}_{1}, \mathbf{y}_{1}\right)+d\left(\mathbf{x}_{2}, \mathbf{y}_{1}\right)+d\left(\mathbf{y}_{1}, \mathbf{y}_{2}\right)+d\left(\mathbf{y}_{2}, \mathbf{x}_{3}\right)+d\left(\mathbf{y}_{2}, \mathbf{x}_{4}\right)
$$

The bounds when $\mathbf{x}_{2}$ or $\mathbf{x}_{3}$ or $\mathbf{x}_{4}$ is fixed instead of $\mathbf{x}_{1}$ are the same.

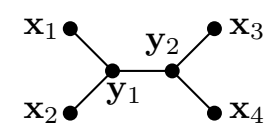

Fig. 1.5 A longer tree

As

$$
\|j(\tau) \mid\| \leq e^{K_{j} \delta} \quad\|j(2 \delta-\tau-\varepsilon)\| \leq e^{2 K_{j} \delta} \quad\|j(\delta-\tau-\varepsilon)\| \leq e^{K_{j} \delta}
$$

and $\alpha^{*}, \beta$ and $z$ have weights $\kappa(2 \delta), \kappa(2 \delta)$ and $4 \mathrm{r}(\delta)$, respectively, we have, for each $\tau \in \varepsilon \mathbb{Z} \cap(0, \delta]$,

$$
\left\|\left\langle\gamma_{* \tau} g_{\tau+\varepsilon}, v \gamma_{* \tau} g_{\tau+\varepsilon}\right\rangle-\left\langle\gamma_{* \tau} \hat{g}_{\tau+\varepsilon}, v \gamma_{* \tau} \hat{g}_{\tau+\varepsilon}\right\rangle\right\|_{\mathrm{fl}} \leq 12 e^{5 K_{j} \delta}\|v\| \mathrm{r}(\delta) \kappa(2 \delta)^{3}
$$

Here, we have assumed that $\Theta \mathfrak{v} \leq \frac{1}{2^{10}}$ so that $4 \mathrm{r}(\delta) \leq \kappa(2 \delta)$. Summing over $\tau$ and multiplying by $\varepsilon$ gives

$$
\left\|\mathcal{V}_{\delta}\left(\varepsilon ; \alpha^{*}, j(\delta) \beta+z\right)-\mathcal{V}_{\delta}\left(\varepsilon ; \alpha^{*}, j(\delta) \beta\right)\right\|_{\mathrm{fl}} \leq 12 e^{5 K_{j} \delta} \delta\|v\| \mathrm{r}(\delta) \kappa(2 \delta)^{3}
$$

Similarly

$$
\left\|\mathcal{V}_{\delta}\left(\varepsilon ; j(\delta) \alpha^{*}+z_{*}, \beta\right)-\mathcal{V}_{\delta}\left(\varepsilon ; j(\delta) \alpha^{*}+z_{*}, \beta\right)\right\|_{\mathrm{fl}} \leq 12 e^{5 K_{j} \delta} \delta\|v\| \mathrm{r}(\delta) \kappa(2 \delta)^{3}
$$

Next, we bound $\mathcal{E}\left(\alpha^{*}, j(\delta) \beta+z\right)-\mathcal{E}\left(\alpha^{*}, j(\delta) \beta\right)$. For any analytic function $f\left(\alpha^{*}, \beta\right)$,

$$
\left\|f\left(\alpha^{*}, j(\delta) \beta+z\right)-f\left(\alpha^{*}, j(\delta) \beta\right)\right\|_{\mathrm{fl}} \leq\left\|f\left(\alpha^{*}, j(\delta) \beta+z\right)\right\|_{\mathrm{fl}}
$$

since the symmetric coefficient system for $f\left(\alpha^{*}, j(\delta) \beta+z\right)-f\left(\alpha^{*}, j(\delta) \beta\right)$ is precisely the symmetric coefficient system for $f\left(\alpha^{*}, j(\delta) \beta+z\right)$, but with the coefficients for terms having no $z$ 's replaced by 0 . So, by Proposition $1.32^{6}$,

$$
\left\|f\left(\alpha^{*}, j(\delta) \beta+z\right)-f\left(\alpha^{*}, j(\delta) \beta\right)\right\|_{\mathrm{fl}} \leq\left\|f\left(\alpha^{*}, j(\delta) \beta+z\right)\right\|_{\mathrm{fl}} \leq\left\|f\left(\alpha^{*}, \beta\right)\right\|_{\delta}
$$

since

$$
\|j(\delta)\| \mid \kappa(2 \delta)+4 \mathrm{r}(\delta) \leq e^{\delta K_{j}} \kappa(2 \delta)+4 \mathrm{r}(\delta)=\left[e^{\delta K_{j}} \frac{1}{2^{\frac{3}{10}}}+4(\delta \mathfrak{v})^{\frac{1}{4}}\right] \kappa(\delta) \leq \kappa(\delta)
$$

if $\Theta$ and $\mathfrak{v}$ are small enough. In particular $\left\|\mathcal{E}\left(\alpha^{*}, j(\delta) \beta+z\right)-\mathcal{E}\left(\alpha^{*}, j(\delta) \beta\right)\right\|_{\mathrm{f}} \leq\|\mathcal{E}\|_{\delta}$. Similarly $\left\|\mathcal{E}\left(j(\delta) \alpha^{*}+z_{*}, \beta\right)-\mathcal{E}\left(j(\delta) \alpha^{*}, \beta\right)\right\|_{\mathrm{fl}} \leq\|\mathcal{E}\|_{\delta}$.

\footnotetext{
${ }^{6}$ Actually, by an obvious generalization of Proposition 1.32, since the $g$ of Proposition 1.32 is a function of a single field. See (Balaban et al., 2010a, Corollary A.2) for such a generalization.
} 
Combining the bounds of the previous two paragraphs and then using the hypothesis that $\|\mathcal{E}\|_{\delta} \leq 4 e^{5 \delta K_{j}} \delta\|v \mid\| \mathrm{r}(\delta) \kappa(2 \delta)^{3}$, we get

$$
\begin{aligned}
\left\|\partial A_{\delta, \varepsilon}(\mathcal{E} ; \cdot)\right\|_{\mathrm{fl}} & \leq 24 e^{5 \delta K_{j}} \delta\|v\| \mathrm{r}(\delta) \kappa(2 \delta)^{3}+2\|\mathcal{E}\|_{\delta} \leq 2^{5} e^{5 \delta K_{j}} \delta\|v\| \| \mathrm{r}(\delta) \kappa(2 \delta)^{3} \\
& \leq 2^{5} e^{5 \delta K_{j}} \frac{1}{2^{\frac{9}{10}}}(\delta \mathfrak{v})^{\frac{1}{20}} \leq \frac{1}{64}
\end{aligned}
$$

if $\Theta \leq 1$ and $\Theta \mathfrak{v}$ is small enough. Finally, by (1.35) and Corollary 1.10

$$
\begin{aligned}
\left\|\log \frac{\int d \mu_{\mathrm{r}(\delta)}\left(z^{*}, z\right) e^{\partial \mathcal{A}_{\delta, \varepsilon}\left(\mathcal{E} ; \alpha^{*}, \beta ; z_{*}, z\right)}}{\int d \mu_{\mathrm{r}(\delta)}\left(z^{*}, z\right)}\right\|_{2 \delta} & \leq \frac{\left\|\partial A_{\delta, \varepsilon}(\mathcal{E} ; \cdot)\right\|_{\mathrm{fl}}^{2}}{\left(\frac{1}{20}-\left\|\partial A_{\delta, \varepsilon}(\mathcal{E} ; \cdot)\right\|_{\mathrm{fl}}\right)^{2}} \\
& \leq 2^{20} e^{10 \delta K_{j}} \delta^{2}\|v\|^{2} \mathrm{r}(\delta)^{2} \kappa(2 \delta)^{6}
\end{aligned}
$$

Combining this estimate and the estimate of Lemma 1.12, below, with $f=\mathcal{E}$, we get the desired bound on $\left\|\Re_{\delta, \varepsilon}[\mathcal{E}]\right\|_{2 \delta}$.

Lemma 1.12 Let $f\left(\alpha^{*}, \beta\right)$ be an analytic function that has degree at least two both in $\alpha^{*}$ and $\beta$. Then

$$
\left\|f\left(\alpha^{*}, j(\delta) \beta\right)\right\|_{2 \delta},\left\|f\left(j(\delta) \alpha^{*}, \beta\right)\right\|_{2 \delta} \leq e^{2 \delta K_{j}}\left(\frac{\kappa(2 \delta)}{\kappa(\delta)}\right)^{4}\|f\|_{\delta}
$$

Proof Introduce the auxiliary norm $\|\cdot\|_{\text {aux }}$ that uses the weight system of mass $m$ that associates the constant weight factor $\kappa(\delta)$ to the field $\alpha_{*}$ and the constant weight factor $e^{-\delta K_{j}} \kappa(\delta)$ to the field $\beta$. Since, by (1.29), $\|j(\delta)\| \mid e^{-\delta K_{j}} \kappa(\delta) \leq \kappa(\delta)$, part (i) of Proposition 1.32 gives

$$
\left\|f\left(\alpha^{*}, j(\delta) \beta\right)\right\|_{w_{\text {aux }}} \leq\|f\|_{\delta}
$$

As $f\left(\alpha^{*}, j(\delta) \beta\right)$ has degree at least two both in $\alpha^{*}$ and $\beta$ and $e^{-\delta K_{j}} \kappa(\delta) \geq \kappa(2 \delta)$, if $\Theta$ is small enough,

$$
\left\|f\left(\alpha^{*}, j(\delta) \beta\right)\right\|_{2 \delta} \leq\left(\frac{\kappa(2 \delta)}{\kappa(\delta)}\right)^{2}\left(\frac{\kappa(2 \delta)}{e^{-\delta K_{j}} \kappa(\delta)}\right)^{2}\left\|f\left(\alpha^{*}, j(\delta) \beta\right)\right\|_{w_{\text {aux }}} \leq e^{2 \delta K_{j}}\left(\frac{\kappa(2 \delta)}{\kappa(\delta)}\right)^{4}\|f\|_{\delta}
$$

The estimate on $\left\|f\left(j(\delta) \alpha^{*}, \beta\right)\right\|_{2 \delta}$ is similar.

Proof of Theorem 1.5 Choose $K_{E}=2^{21} e^{10 K_{j}}$. We write $\delta=\varepsilon_{n}=2^{n} \varepsilon$ and prove the statement by induction on $n$. In the case $n=0$ there is nothing to prove. For the induction step from $n$ to $n+1$, set $\delta=\varepsilon_{n}$. The hypothesis of Proposition 1.11, with $\mathcal{E}=\mathcal{E}_{\delta}$, is satisfied since, by the inductive hypothesis,

$$
\left\|\mathcal{E}_{\delta}\right\|_{\delta} \leq K_{E} \delta^{2}\|v\|^{2} \mathrm{r}(\delta)^{2} \kappa(\delta)^{6}=K_{E}(\delta \mathfrak{v})^{\frac{1}{20}} 2^{\frac{9}{10}} \delta\|v\|\left\|\mathrm{r}(\delta) \kappa(2 \delta)^{3} \leq \delta\right\| v \| \mathrm{r}(\delta) \kappa(2 \delta)^{3}
$$

if $\Theta \mathfrak{v}$ has been chosen small enough. Using (1.31) and Proposition 1.11, we see that 


$$
\begin{aligned}
\left\|\mathcal{E}_{\varepsilon_{n+1}}\right\|_{\varepsilon_{n+1}} & \leq 2^{20} e^{10 \delta K_{j}} \delta^{2}\|v\|^{2} \mathrm{r}(\delta)^{2} \kappa(2 \delta)^{6}+2 e^{2 \delta K_{j}}\left(\frac{\kappa(2 \delta)}{\kappa(\delta)}\right)^{4} K_{E} \delta^{2}\|v\| \|^{2} \mathrm{r}(\delta)^{2} \kappa(\delta)^{6} \\
& =\left[2^{20} e^{10 \delta K_{j}}+2 e^{2 \delta K_{j}}\left(\frac{\kappa(\delta)}{\kappa(2 \delta)}\right)^{2} K_{E}\right] \delta^{2}\|v\|^{2} \mathrm{r}(\delta)^{2} \kappa(2 \delta)^{6} \\
& =\frac{1}{2}\left[\frac{2^{19} e^{10 \delta K_{j}}}{K_{E}}+e^{2 \delta K_{j}}\left(\frac{\kappa(\delta)}{\kappa(2 \delta)}\right)^{2}\right]\left(\frac{\mathrm{r}(\delta)}{\mathrm{r}(2 \delta)}\right)^{2} K_{E}(2 \delta)^{2}\|v\|^{2} \mathrm{r}(2 \delta)^{2} \kappa(2 \delta)^{6} \\
& =\frac{1}{2}\left[\frac{1}{4}+e^{2 \delta K_{j}} 2^{\frac{6}{10}}\right] 2^{\frac{1}{10}} K_{E}(2 \delta)^{2}\|v\|^{2} \mathrm{r}(2 \delta)^{2} \kappa(2 \delta)^{6} \\
& \leq K_{E}(2 \delta)^{2}\|v\|^{2} \mathrm{r}(2 \delta)^{2} \kappa(2 \delta)^{6} \quad(\text { if } \Theta \text { has been chosen small enough) } \\
& =K_{E} \varepsilon_{n+1}^{2}\|v\|^{2} \mathrm{r}\left(\varepsilon_{n+1}\right)^{2} \kappa\left(\varepsilon_{n+1}\right)^{6}
\end{aligned}
$$

\subsection{Functional Integrals}

In this section, I will outline the proof of a functional integral representation of the partition function like that of Theorem 1.1. It is an example of the class of rigorous functional integral representations in which the object of interest is expressed as a limit of finite dimensional integrals. At the end of this section, I will mention, and provide references to, a couple of other classes of rigorous functional integral representations that are used in mathematical physics.

I remind you that we have decided to approximate the left hand side of (1.1) by replacing space $\mathbb{R}^{3}$ by a finite number of points, say $X=\mathbb{Z}^{3} / L \mathbb{Z}^{3}$ and that the Hamiltonian is

$$
H=\int d \mathbf{x} d \mathbf{y} \psi^{\dagger}(\mathbf{x}) \mathrm{h}(\mathbf{x}, \mathbf{y}) \psi(\mathbf{y})+\int d \mathbf{x}_{1} d \mathbf{x}_{2} \psi^{\dagger}\left(\mathbf{x}_{1}\right) \psi^{\dagger}\left(\mathbf{x}_{2}\right) v\left(\mathbf{x}_{1}, \mathbf{x}_{2}\right) \psi\left(\mathbf{x}_{1}\right) \psi\left(\mathbf{x}_{2}\right)
$$

with $\int d \mathbf{x}$ just meaning $\sum_{\mathbf{x} \in X}$. We are still assuming that the kinetic energy operator $h \geq 0$ and that the two-dody potential $2 v(\mathbf{x}, \mathbf{y})$ is strictly positive when viewed as the kernel of an integral operator. I have claimed that then the partition function is (pretty obviously) well-defined. Let's check that this is indeed the case. The Hilbert space of all states of this system is

$$
\mathcal{F}=\bigoplus_{n=0}^{\infty} \mathcal{F}_{n} \text { with } \mathcal{F}_{n}=L_{s}^{2}\left(X^{n}\right)=\mathbb{C}^{|X|^{n}} / S_{n}
$$

Here

- a vector in the $n$-particle subspace $\mathcal{F}_{n}$ is a function $f\left(\mathbf{x}_{1}, \cdots, \mathbf{x}_{n}\right)$, with each argument $\mathbf{x}_{j}$ running over $X$, that is invariant under permutation of its arguments

- the inner product between two $n$-particle vectors $f, g \in \mathcal{F}_{n}$ is

$$
\langle f, g\rangle_{\mathcal{F}_{n}}=\int_{X^{n}} d \mathbf{x}_{1} \cdots d \mathbf{x}_{n} \overline{f\left(\mathbf{x}_{1}, \cdots, \mathbf{x}_{n}\right)} g\left(\mathbf{x}_{1}, \cdots, \mathbf{x}_{n}\right)
$$

where $\int_{X} d \mathbf{x} f(\mathbf{x})$ just means $\sum_{\mathbf{x} \in X} f(\mathbf{x})$ 
- $\mathcal{F}_{0}=\mathbb{C}$

- the inner product between two vectors $\mathbf{f}=\left(f_{n}\right)_{n \geq 0}$ and $\mathbf{g}=\left(g_{n}\right)_{n \geq 0}$ in $\mathcal{F}$ is

$$
\langle\mathbf{f}, \mathbf{g}\rangle_{\mathcal{F}}=\sum_{n \geq 0}\left\langle f_{n}, g_{n}\right\rangle_{\mathcal{F}_{n}}
$$

Now both $H$ and $N$ map the $n$-particle space $\mathcal{F}_{n}$ (which is finite dimensional) into itself. We'll show that the, positive, operator $e^{-\frac{1}{k T}(H-\mu N)}$ is trace class by bounding, for each nonnegative integer $n$, the trace of the restriction of $e^{-\frac{1}{k T}(H-\mu N)}$ to $\mathcal{F}_{n}$ and then observe that the bound is easily summable over $n$. Now the restriction of $N$ to $\mathcal{F}_{n}$ is just $n \mathbb{1}$ and the following lemma provides a lower bound on $H \nmid \mathcal{F}_{n}$.

Lemma 1.13 There are constants $C, D>0$ such that the restriction of $H$ to $\mathcal{F}_{n}$ is bounded below by $(C n-D) n 1$.

Proof Use $\psi^{\dagger}(\mathbf{x}), \psi(\mathbf{x})$ to denote the annihilation and creation operators at $\mathbf{x} \in X$. By the commutation relations $\left[\psi(\mathbf{x}), \psi^{\dagger}\left(\mathbf{x}^{\prime}\right)\right]=\delta_{\mathbf{x}, \mathbf{x}^{\prime}}$, the interaction

$$
\begin{aligned}
V & =\int d \mathbf{x}_{1} d \mathbf{x}_{2} \psi^{\dagger}\left(\mathbf{x}_{1}\right) \psi^{\dagger}\left(\mathbf{x}_{2}\right) v\left(\mathbf{x}_{1}, \mathbf{x}_{2}\right) \psi\left(\mathbf{x}_{1}\right) \psi\left(\mathbf{x}_{2}\right) \\
& =\int d \mathbf{x}_{1} d \mathbf{x}_{2} \psi^{\dagger}\left(\mathbf{x}_{1}\right) \psi\left(\mathbf{x}_{1}\right) v\left(\mathbf{x}_{1}, \mathbf{x}_{2}\right) \psi^{\dagger}\left(\mathbf{x}_{2}\right) \psi\left(\mathbf{x}_{2}\right)-\int d \mathbf{x} \psi^{\dagger}(\mathbf{x}) v(\mathbf{x}, \mathbf{x}) \psi(\mathbf{x}) \\
& =\int d \mathbf{x}_{1} d \mathbf{x}_{2} n\left(\mathbf{x}_{1}\right) v\left(\mathbf{x}_{1}, \mathbf{x}_{2}\right) n\left(\mathbf{x}_{2}\right)-\int d \mathbf{x} v(\mathbf{x}, \mathbf{x}) n(\mathbf{x})
\end{aligned}
$$

where $n(\mathbf{x})=\psi^{\dagger}(\mathbf{x}) \psi(\mathbf{x})$ is the local number operator at $\mathbf{x}$. Now, restricted to $\mathcal{F}_{n}$, $\{n(\mathbf{x}) \mid \mathbf{x} \in X\}$ is a family of commuting, bounded self-adjoint operators on the finite dimensional Hilbert space $\mathcal{F}_{n}$ (that is, they are self-adjoint matrices). So there is an orthonormal basis $\left\{\delta_{Y}\right\}$ for $\mathcal{F}_{n}$ consisting of simultaneous eigenvectors for all of the $n(\mathbf{x})$ 's. We denote the eigenvalues $\mu_{Y}(\mathbf{x})$. (All this is easy to find and is given in (Balaban et al., 2008a), but we don't need the explicit formulae.) So, for any $\varphi=$ $\sum_{Y} \varphi_{Y} \delta_{Y}$

$$
\begin{aligned}
\langle\varphi, & \left.\int_{X^{2}} d \mathbf{x}_{1} d \mathbf{x}_{2} n\left(\mathbf{x}_{1}\right) v\left(\mathbf{x}_{1}, \mathbf{x}_{2}\right) n\left(\mathbf{x}_{2}\right) \varphi\right\rangle \\
& =\sum_{Y_{1}, Y_{2}} \overline{\varphi_{Y_{1}}} \varphi_{Y_{2}}\left\langle\delta_{Y_{1}}, \int_{X^{2}} d \mathbf{x}_{1} d \mathbf{x}_{2} n\left(\mathbf{x}_{1}\right) v\left(\mathbf{x}_{1}, \mathbf{x}_{2}\right) n\left(\mathbf{x}_{2}\right) \delta_{Y_{2}}\right\rangle \\
& =\int_{X^{2}} d \mathbf{x}_{1} d \mathbf{x}_{2} v\left(\mathbf{x}_{1}, \mathbf{x}_{2}\right) \sum_{Y_{1}, Y_{2}} \overline{\varphi_{Y_{1}}} \varphi_{Y_{2}}\left\langle n\left(\mathbf{x}_{1}\right) \delta_{Y_{1}}, n\left(\mathbf{x}_{2}\right) \delta_{Y_{2}}\right\rangle \\
& =\int_{X^{2}} d \mathbf{x}_{1} d \mathbf{x}_{2} v\left(\mathbf{x}_{1}, \mathbf{x}_{2}\right) \sum_{Y_{1}, Y_{2}} \overline{\varphi_{Y_{1}}} \varphi_{Y_{2}} \mu_{Y_{1}}\left(\mathbf{x}_{1}\right) \mu_{Y_{2}}\left(\mathbf{x}_{2}\right)\left\langle\delta_{Y_{1}}, \delta_{Y_{2}}\right\rangle \\
& =\sum_{Y}\left|\varphi_{Y}\right|^{2} \int_{X^{2}} d \mathbf{x}_{1} d \mathbf{x}_{2} \mu_{Y}\left(\mathbf{x}_{1}\right) v\left(\mathbf{x}_{1}, \mathbf{x}_{2}\right) \mu_{Y}\left(\mathbf{x}_{2}\right)
\end{aligned}
$$




\section{The Temporal Ultraviolet Limit}

By hypothesis, $v$ is a strictly positive operator on $L^{2}(X)$. Denote by $\lambda_{0}>0$ its smallest eigenvalue. Then

$$
\begin{aligned}
\int_{X^{2}} d \mathbf{x}_{1} d \mathbf{x}_{2} \mu_{Y}\left(\mathbf{x}_{1}\right) v\left(\mathbf{x}_{1}, \mathbf{x}_{2}\right) \mu_{Y}\left(\mathbf{x}_{2}\right) & \geq \lambda_{0} \int_{X} d \mathbf{x} \mu_{Y}^{2}(\mathbf{x}) \geq \frac{\lambda_{0}}{|X|}\left(\int_{X} d \mathbf{x} \mu_{Y}(\mathbf{x})\right)^{2} \\
& =\frac{\lambda_{0}}{|X|} n^{2}
\end{aligned}
$$

by Cauchy-Schwartz and the fact that, on $\mathcal{F}_{n}, \int_{X} d \mathbf{x} n(\mathbf{x})=n$. Hence

$$
\left\langle\varphi, \int_{X^{2}} d \mathbf{x}_{1} d \mathbf{x}_{2} n\left(\mathbf{x}_{1}\right) v\left(\mathbf{x}_{1}, \mathbf{x}_{2}\right) n\left(\mathbf{x}_{2}\right) \varphi\right\rangle \geq \frac{\lambda_{0}}{|X|} n^{2} \sum_{Y}\left|\varphi_{Y}\right|^{2}=\frac{\lambda_{0}}{|X|} n^{2}\|\varphi\|^{2}
$$

Since, on $\mathcal{F}_{n}$ the $n(\mathbf{x})^{\text {'s }}$ are positive operators adding up to $n$, every $0 \leq \mu_{Y}(\mathbf{x}) \leq n$ and

$$
\begin{aligned}
& \|\psi(\mathbf{x})\|_{\mathcal{F}_{n} \rightarrow \mathcal{F}_{n-1}}^{2}=\left\|\psi(\mathbf{x})^{\dagger} \psi(\mathbf{x})\right\|_{\mathcal{F}_{n}}=\|n(\mathbf{x})\|_{\mathcal{F}_{n}} \leq n \\
& \left\|\psi(\mathbf{x})^{\dagger}\right\|_{\mathcal{F}_{n-1} \rightarrow \mathcal{F}_{n}}=\|\psi(\mathbf{x})\|_{\mathcal{F}_{n} \rightarrow \mathcal{F}_{n-1}} \leq \sqrt{n}
\end{aligned}
$$

Consequently

$$
\left\|H_{0}\right\|=\left\|\int d \mathbf{x} d \mathbf{y} \psi^{\dagger}(\mathbf{x}) \mathrm{h}(\mathbf{x}, \mathbf{y}) \psi(\mathbf{y})\right\| \leq n \int d \mathbf{x} d \mathbf{y}|\mathrm{h}(\mathbf{x}, \mathbf{y})|
$$

We can easily do better than this, but we don't need to. The lemma follows with $C=\frac{\lambda_{0}}{|X|}$.

Now back to the trace. Since the dimension of $\mathcal{F}_{n}$ is less than $|X|^{n}$ and every eigenvalue of $(H-\mu N) \uparrow \mathcal{F}_{n}$ is at least $C n^{2}-D n-\mu n$, we have

$$
\operatorname{Tr}_{\mathcal{F}_{n}} e^{-\frac{1}{k T}(H-\mu N)} \leq e^{-\frac{1}{k T}\left(C n^{2}-D n-\mu n\right)}|X|^{n}
$$

This is obviously summable over $n$.

\subsubsection{A Rigorous Version of the Functional Integral}

So we now know that, when $X$ is finite, the partition function $\operatorname{Tr}_{\mathcal{F}} e^{-\frac{1}{k T}(H-\mu N)}$ is well-defined. I'll now outline the proof of a functional integral representation for $\operatorname{Tr}_{\mathcal{F}} e^{-\frac{1}{k T}(H-\mu N)}$ that is similar to that of Theorem 1.1, but whose integrand looks a lot more like the $e^{\mathcal{A}\left(\alpha^{*}, \alpha\right)}$ with the $\mathcal{A}\left(\alpha^{*}, \alpha\right)$ of (1.2).

We use the notation $\beta=\frac{1}{k T}$ and, for any $p \in \mathbb{N}$,

$$
\begin{aligned}
\mathcal{T}_{p}= & \left\{\tau=q \frac{\beta}{p} \mid q=1, \cdots, p\right\} \\
\varepsilon_{p}= & \frac{\beta}{p} \\
d \mu_{p, r}\left(\alpha^{*}, \alpha\right)= & \prod_{\tau \in \mathcal{T}_{p}} \prod_{\mathbf{x} \in X}\left[\frac{d \alpha_{\tau}^{*}(\mathbf{x}) \wedge d \alpha_{\tau}(\mathbf{x})}{2 \pi \imath} \chi\left(\left|\alpha_{\tau}(\mathbf{x})\right|<r\right)\right] \\
K\left(\alpha^{*}, \alpha\right)= & \iint d \mathbf{x} d \mathbf{y} \alpha(\mathbf{x})^{*} h(\mathbf{x}, \mathbf{y}) \alpha(\mathbf{y})-\mu \int d \mathbf{x} \alpha(\mathbf{x})^{*} \alpha(\mathbf{x}) \\
& +\iint d \mathbf{x} d \mathbf{y} \alpha(\mathbf{x})^{*} \alpha(\mathbf{x}) v(\mathbf{x}, \mathbf{y}) \alpha(\mathbf{y})^{*} \alpha(\mathbf{y})
\end{aligned}
$$


Theorem 1.14 Suppose that the sequence $\mathrm{R}(p) \rightarrow \infty$ as $p \rightarrow \infty$ at a suitable rate. Precisely

$$
\lim _{p \rightarrow \infty} p e^{-\frac{1}{2} \mathrm{R}(p)^{2}}=0 \quad \text { and } \quad \mathrm{R}(p)<p^{\frac{1}{24|X|}}
$$

Then

$\operatorname{Tr} e^{-\beta(H-\mu N)}=\lim _{p \rightarrow \infty} \int d \mu_{p, \mathrm{R}(p)}\left(\alpha^{*}, \alpha\right) \prod_{\tau \in \mathcal{T}_{p}} e^{-\int d \mathbf{y}\left[\alpha_{\tau}^{*}(\mathbf{y})-\alpha_{\tau-\varepsilon_{p}}^{*}(\mathbf{y})\right] \alpha_{\tau}(\mathbf{y})} e^{-\varepsilon_{p} K\left(\alpha_{\tau-\varepsilon_{p}}^{*}, \alpha_{\tau}\right)}$ with the convention that $\alpha_{0}=\alpha_{\beta}$.

Almost all of the rest of this section is used to outline the proof of Theorem 1.14.

\subsubsection{The Main Ingredients - Coherent States}

The first main ingredient in the proof is the use of coherent states. I'll give the formulae only for the case $|X|=1$, because then they are short and clean. The general case is very similar. If $|X|=1$, then

$$
\mathcal{F}=\bigoplus_{n=0}^{\infty} \mathcal{F}_{n} \text { with } \mathcal{F}_{n}=\mathbb{C}
$$

Let $e_{n}=1 \in \mathbb{C}=\mathcal{F}_{n}$. We can think of each vector in $\mathcal{F}$ as a sequence $\left(v_{0}, v_{1}, v_{2}, \cdots\right)$ of complex numbers. Then $e_{n}$ is the sequence all of whose components are zero, except for that with index $n$, which is 1 . For each $\alpha \in \mathbb{C}$ the coherent state

$$
|\alpha\rangle=\sum_{n=0}^{\infty} \frac{1}{\sqrt{n !}} \alpha^{n} e_{n} \in \mathcal{F}
$$

is an eigenvector for the field (or annihilation) operator

$$
\psi e_{n}=\sqrt{n} e_{n-1}
$$

To check this, we compute

$$
\psi|\alpha\rangle=\sum_{n=1}^{\infty} \frac{1}{\sqrt{(n-1) !}} \alpha^{n} e_{n-1}=\alpha|\alpha\rangle
$$

The action of the creation operator

$$
\psi^{\dagger} e_{n}=\sqrt{n+1} e_{n+1}
$$

on the $\alpha^{\text {th }}$ coherent state vector is

$$
\psi^{\dagger}|\alpha\rangle=\sum_{n=0}^{\infty} \frac{\sqrt{n+1}}{\sqrt{n !}} \alpha^{n} e_{n+1}=\frac{\partial}{\partial \alpha} \sum_{n=0}^{\infty} \frac{1}{\sqrt{(n+1) !}} \alpha^{n+1} e_{n+1}=\frac{\partial}{\partial \alpha}|\alpha\rangle
$$




\section{The Temporal Ultraviolet Limit}

Because the $e_{n}$ 's form an orthonormal basis, the inner product between two coherent states is

$$
\langle\alpha \mid \gamma\rangle=\sum_{m, n=0}^{\infty} \frac{\bar{\alpha}^{m}}{\sqrt{m !}} \frac{\gamma^{n}}{\sqrt{n !}} \delta_{m, n}=\sum_{n=0}^{\infty} \frac{1}{n !}(\bar{\alpha} \gamma)^{n}=e^{\bar{\alpha} \gamma}
$$

For general $X$, there is a similar coherent state $|\alpha\rangle$ for each $|X|$-component complex vector $\alpha \in \mathbb{C}^{|X|}$. The inner product between two such coherent states is

$$
\langle\alpha \mid \gamma\rangle=e^{\int d \mathbf{y} \overline{\alpha(\mathbf{y})} \gamma(\mathbf{y})}
$$

\subsubsection{The Main Ingredients - Approximate Resolution of the Identity}

One of our main tools is going to be the analog for coherent states of the identity that for any orthonormal basis

$$
11 v=\sum_{n}\left(e_{n}, v\right) e_{n}
$$

Formally, the corresponding statement for coherent states is

$$
\mathbb{1}=\int \prod_{\mathbf{x} \in X}\left[\frac{d \alpha^{*}(\mathbf{x}) \wedge d \alpha(\mathbf{x})}{2 \pi \imath}\right] e^{-\int d \mathbf{y}|\alpha(\mathbf{y})|^{2}}|\alpha\rangle\langle\alpha|
$$

where $|\alpha\rangle\langle\alpha|$ is the linear operator that maps $v \in \mathcal{F}$ to the inner product of $v$ and $|\alpha\rangle$ times the vector $|\alpha\rangle$. The integral "sums" over all possible coherent states and the exponential $e^{-\int d \mathbf{y}|\alpha(\mathbf{y})|^{2}}=\frac{1}{\||\alpha\rangle \|^{2}}$ turns the coherent states into unit vectors. Here is a rigorous version of the resolution of the identity for coherent states.

Theorem 1.15 For each $r>0$, let

$$
\mathrm{I}_{r}=\prod_{\mathbf{x} \in X}\left[\int_{|\alpha(\mathbf{x})|<r} \frac{d \alpha^{*}(\mathbf{x}) \wedge d \alpha(\mathbf{x})}{2 \pi \imath}\right] e^{-\int d \mathbf{y}|\alpha(\mathbf{y})|^{2}}|\alpha\rangle\langle\alpha|
$$

Then

(a) $0<\mathrm{I}_{r}<1$.

(b) $\mathrm{I}_{r}$ commutes with $N$.

(c) The operator norm of $\mathrm{I}_{r}$ is bounded by one for all $r$ and, for each vector $v \in \mathcal{F}$, $\mathrm{I}_{r} v$ converges to $v$ as $r \rightarrow \infty$. That is, $\mathrm{I}_{r}$ convergences strongly to 1 .

(d) For all $n$ and $r$, the operator norms

$$
\left\|\left(\mathbb{1}-\mathrm{I}_{r}\right) \uparrow \mathcal{F}_{n}\right\| \leq|X| 2^{n+1} e^{-r^{2} / 2} \quad\left\|\mathrm{I}_{r} \uparrow \mathcal{F}_{n}\right\| \leq \frac{1}{n !}\left(|X| r^{2}\right)^{n}
$$

Remark 1.16 Observe, from part $(d)$, that if $\frac{n}{r^{2}} \ll 1$ then $\mathrm{I}_{r} \uparrow \mathcal{F}_{n} \approx \mathbb{1}$ while if $\frac{n}{r^{2}} \gg 1$, then $\mathrm{I}_{r} \uparrow \mathcal{F}_{n} \approx 0$ (use that $n ! \approx n^{n}$ ). So we can think of $\mathrm{I}_{r}$ as being, very roughly, projection onto $\bigoplus_{n=0}^{r^{2}} \mathcal{F}_{n}$. 
Proof We first observe that when you apply the operator $e^{-\int d \mathbf{y}|\alpha(\mathbf{y})|^{2}}|\alpha\rangle\langle\alpha|$ to some vector $v$, the resulting vector is of norm at most $\frac{1}{\| \alpha\rangle \|^{2}} \||\alpha\rangle\|\||\alpha\rangle\|\| v\|\leq\| v \|$. So integrand of the right hand side of (1.44) is of operator norm at most one. As it is also continuous in $\alpha$ and the domain of integration is of finite volume, the right hand side of (1.44) is obviously well-defined.

The proof is easy once one has an orthonormal basis of eigenvectors for $\mathrm{I}_{r}$ - and it is easy to just guess such a basis. Again, to simplify the notation, I'll just give the proof for $|X|=1$. Then

$$
\mathcal{F}=\bigoplus_{n=0}^{\infty} \mathcal{F}_{n} \text { with } \mathcal{F}_{n}=\mathbb{C}
$$

and $\left\{e_{m}=1 \in \mathbb{C}=\mathcal{F}_{m} \mid m=0,1,2,3, \cdots\right\}$ is an orthonormal basis for $\mathcal{F}$. If part (b) of the Theorem is true, then each of the $\mathcal{F}_{m}$ 's, which has basis $\left\{e_{m}\right\}$, will be left invariant by $\mathrm{I}_{r}$. So each $e_{m}$ will be an eigenvector. To verify that this is indeed the case, and to find the corresponding eigenvalues, we compute $\mathrm{I}_{r} e_{m}$.

Recall that

$$
|\alpha\rangle=\sum_{n=0}^{\infty} \frac{1}{\sqrt{n !}} \alpha^{n} e_{n}
$$

So

$$
\begin{aligned}
\mathrm{I}_{r} e_{m} & =\int_{|\alpha|<r} \frac{d \bar{\alpha} \wedge d \alpha}{2 \pi \imath} e^{-|\alpha|^{2}}|\alpha\rangle\left\langle\alpha \mid e_{m}\right\rangle=\int_{|\alpha|<r} \frac{d \bar{\alpha} \wedge d \alpha}{2 \pi \imath} e^{-|\alpha|^{2}}|\alpha\rangle \frac{1}{\sqrt{m !}} \bar{\alpha}^{m} \\
& =\sum_{n=0}^{\infty} \frac{1}{\sqrt{n !} \sqrt{m !}} e_{n} \int_{|\alpha|<r} \frac{d \bar{\alpha} \wedge d \alpha}{2 \pi \imath} e^{-|\alpha|^{2}} \bar{\alpha}^{m} \alpha^{n}
\end{aligned}
$$

Now switch to polar coordinates. That is, make the change of variables $\alpha=\rho e^{i \theta}$. Recalling that if $z=x+i y$, then $\frac{d \bar{z} \wedge d z}{2 \pi i}=\frac{d x \wedge d y}{\pi}$,

$$
\begin{aligned}
\mathrm{I}_{r} e_{m} & =\sum_{n=0}^{\infty} \frac{1}{\sqrt{n !} \sqrt{m !}} e_{n} \int_{0}^{2 \pi} d \theta \int_{0}^{r} d \rho \frac{1}{\pi} e^{-\rho^{2}} \rho^{m+n+1} e^{i \theta(n-m)} \\
& =\frac{1}{m !} e_{m} \int_{0}^{r} d \rho 2 e^{-\rho^{2}} \rho^{2 m+1}=\frac{1}{m !} e_{m} \int_{0}^{r^{2}} d t e^{-t} t^{m} \quad \text { where } t=\rho^{2} \\
& =\left\{1-\frac{1}{m !} \int_{r^{2}}^{\infty} e^{-t} t^{m} d t\right\} e_{m}
\end{aligned}
$$

This tells us that each $e_{m}$ is an eigenvector of $\mathrm{I}_{r}$ with eigenvalue $1-\frac{1}{m !} \int_{r^{2}}^{\infty} e^{-t} t^{m} d t$, which is always between 0 and 1 and which tends to zero as $r \rightarrow \infty$. Parts (a), (b) and (c) follow. For part (d), just bound

$$
\frac{1}{n !} \int_{r^{2}}^{\infty} e^{-t} t^{n} d t=2^{n} \int_{r^{2}}^{\infty} e^{-t} \frac{1}{n !}\left(\frac{t}{2}\right)^{n} d t \leq 2^{n} \int_{r^{2}}^{\infty} e^{-t} e^{t / 2} d t=2^{n+1} e^{-r^{2} / 2}
$$

and

$$
\frac{1}{n !} \int_{0}^{r^{2}} e^{-t} t^{n} d t \leq \frac{1}{n !} r^{2 n} \int_{0}^{\infty} e^{-t} d t=\frac{1}{n !} r^{2 n}
$$




\section{The Temporal Ultraviolet Limit}

\subsubsection{The Main Ingredients - Trace}

Formally, the analog of

$$
\operatorname{Tr} B=\sum_{n}\left(e_{n}, B e_{n}\right)
$$

for coherent states is

$$
\operatorname{Tr} B=\int \prod_{\mathbf{x} \in X}\left[\frac{d \alpha^{*}(\mathbf{x}) d \alpha(\mathbf{x})}{2 \pi \imath}\right] e^{-\int d \mathbf{y}|\alpha(\mathbf{y})|^{2}}\langle\alpha|B| \alpha\rangle
$$

Our next main tool for the proof is the following rigorous version of that formula.

Proposition 1.17 Let $B$ be a bounded operator on $\mathcal{F}$. For all $r>0, B \mathrm{I}_{r}$ is trace class and

$$
\operatorname{Tr} B \mathrm{I}_{r}=\prod_{\mathbf{x} \in X}\left[\int_{|\alpha(\mathbf{x})|<r} \frac{d \alpha^{*}(\mathbf{x}) d \alpha(\mathbf{x})}{2 \pi \imath}\right] e^{-\int d \mathbf{y}|\alpha(\mathbf{y})|^{2}}\langle\alpha|B| \alpha\rangle
$$

Proof As usual, I'll just give the proof for $|X|=1$.

Recall that, by definition, $B \mathrm{I}_{r}$ is trace class when the eigenvalues of the operator square root of $\mathrm{I}_{r} B^{*} B \mathrm{I}_{r}$ (all of which are nonnegative) are summable. There is a theorem which says that a product of a trace class operator (in our case $\mathrm{I}_{r}$ ) and a bounded operator (in our case $B$ ) is trace class. In our case, we can also easily check directly that $B \mathrm{I}_{r}$ is trace class. Here is the argument. By the min-max principle (Reed and Simon, 1978, Theorem XIII.1, with $\left.H=-I_{r} B^{*} B \mathrm{I}_{r}\right)$ the $(n+1)^{\text {st }}$ eigenvalue of $\mathrm{I}_{r} B^{*} B \mathrm{I}_{r}$, counting from largest to smallest, is

$$
\begin{aligned}
\inf _{\varphi_{1}, \cdots \varphi_{n} \in \mathcal{F}} \sup _{\substack{\psi \in \mathcal{F},\|\psi\|=1 \\
\psi \perp \varphi_{1}, \cdots, \varphi_{n}}}\left\langle\psi\left|\mathrm{I}_{r} B^{*} B \mathrm{I}_{r}\right| \psi\right\rangle & \leq \sup _{\substack{\psi \in \oplus_{m \geq n} \mathcal{F}_{m} \\
\|\psi\|=1}}\left\langle\psi\left|\mathrm{I}_{r} B^{*} B \mathrm{I}_{r}\right| \psi\right\rangle \\
& \leq\|B\|^{2} \sup _{m \geq n}\left(\frac{1}{m !} r^{2 m}\right)^{2}
\end{aligned}
$$

by part $(\mathrm{d})$ of Theorem 1.15 . Hence the $(n+1)^{\text {st }}$ eigenvalue of the operator square root, again counting from largest to smallest, is at most $\|B\| \sup _{m \geq n} \frac{1}{m !} r^{2 m}$ and this is clearly summable over $n$.

So $B \mathrm{I}_{r}$ is trace class and the trace itself is

$$
\begin{aligned}
\operatorname{Tr} B \mathrm{I}_{r} & =\sum_{m}\left\langle e_{m}\left|B \mathrm{I}_{r}\right| e_{m}\right\rangle \\
& =\sum_{m} \int_{|\alpha|<r} \frac{d \bar{\alpha} \wedge d \alpha}{2 \pi \imath} e^{-|\alpha|^{2}}\left\langle e_{m}|B| \alpha\right\rangle\left\langle\alpha \mid e_{m}\right\rangle \\
& =\int_{|\alpha|<r} \frac{d \bar{\alpha} \wedge d \alpha}{2 \pi \imath} e^{-|\alpha|^{2}} \sum_{m}\left\langle\alpha \mid e_{m}\right\rangle\left\langle e_{m}|B| \alpha\right\rangle \\
& =\int_{|\alpha|<r} \frac{d \bar{\alpha} \wedge d \alpha}{2 \pi \imath} e^{-|\alpha|^{2}}\langle\alpha|B| \alpha\rangle
\end{aligned}
$$


Moving the sum over $m$ inside the integral is justified by the Lebesgue dominated convergence theorem, since

$$
\sum_{m}\left|\left\langle\alpha \mid e_{m}\right\rangle\left\langle e_{m}|B| \alpha\right\rangle\right| \leq \||\alpha\rangle\|\| B|\alpha\rangle\|\leq\| B \| e^{|\alpha|^{2}}
$$

\subsubsection{Consolidation - Where We Are Now}

Combining Theorem 1.15 and Proposition 1.17, we now have

Lemma 1.18 Assume that $\lim _{p \rightarrow \infty} p e^{-\frac{1}{2} \mathrm{R}(p)^{2}}=0$. Then

$\operatorname{Tr} e^{-\beta(H-\mu N)}=\lim _{p \rightarrow \infty} \prod_{\substack{\mathbf{x} \in X \\ \tau \in \mathcal{T}_{p}}}\left[\int \frac{d \alpha_{\tau}^{*}(\mathbf{x}) d \alpha_{\tau}(\mathbf{x})}{2 \pi \imath} e^{-\left|\alpha_{\tau}(\mathbf{x})\right|^{2}}\right] \prod_{\left|\alpha_{\tau}(\mathbf{x})\right|<\mathrm{R}(p)}\left\langle\alpha_{\tau-\frac{\beta}{p}}\left|e^{-\frac{\beta}{p}(H-\mu N)}\right| \alpha_{\tau}\right\rangle$

Proof By Theorem 1.15,

$$
\operatorname{Tr} e^{-\beta(H-\mu N)}=\operatorname{Tr} \prod_{\tau \in \mathcal{T}_{p}} e^{-\frac{\beta}{p}(H-\mu N)} \mathbb{1}=\lim _{p \rightarrow \infty} \operatorname{Tr} \prod_{\tau \in \mathcal{T}_{p}} e^{-\frac{\beta}{p}(H-\mu N)} \mathrm{I}_{\mathrm{R}(p)}
$$

To justify the last step

- Let $\varepsilon>0$.

- Denote by $P_{n}$ the orthogonal projector onto $\oplus_{m=0}^{n} \mathcal{F}_{n}$. Use Lemma 1.13 to select an $n \in \mathbb{N}$, independent of $p$, such that

$$
\left|\operatorname{Tr}\left(\mathbb{1}-P_{n}\right) \prod_{\tau \in \mathcal{T}_{p}} e^{-\frac{\beta}{p}(H-\mu N)} \mathbb{1}\right|<\frac{\varepsilon}{4} \quad\left|\operatorname{Tr}\left(\mathbb{1}-P_{n}\right) \prod_{\tau \in \mathcal{T}_{p}} e^{-\frac{\beta}{p}(H-\mu N)} \mathrm{I}_{\mathrm{R}(p)}\right|<\frac{\varepsilon}{4}
$$

- Express

$$
P_{n} \prod_{\tau \in \mathcal{T}_{p}} e^{-\frac{\beta}{p}(H-\mu N)} \mathbb{1}-P_{n} \prod_{\tau \in \mathcal{T}_{p}} e^{-\frac{\beta}{p}(H-\mu N)} \mathrm{I}_{\mathrm{R}(p)}
$$

as the telescoping sum over $1 \leq \ell \leq p$ of

$$
P_{n} \prod_{\tau \in \mathcal{T}_{p}} e^{-\frac{\beta}{p}(H-\mu N)} I_{\tau}^{\ell} \quad \text { with } I_{\tau}^{\ell}= \begin{cases}1 & \text { if } \tau<\ell \frac{\beta}{p} \\ 1-\mathrm{I}_{\mathrm{R}(p)} & \text { if } \tau=\ell \frac{\beta}{p} \\ \mathrm{I}_{\mathrm{R}(p)} & \text { if } \tau>\ell \frac{\beta}{p}\end{cases}
$$

- By Lemma 1.13 and part (d) of Theorem 1.15, the trace of each of the $p$ terms in the telescoping sum is bounded by a constant $C$, which depends on $|X|$ and $n$, times $e^{-\mathrm{R}(p)^{2} / 2}$.

- Finally, by hypothesis, if $p$ is sufficiently large then $C p e^{-\mathrm{R}(p)^{2} / 2}<\frac{\varepsilon}{2}$.

It now suffices to substitute in the definition (1.44) of $\mathrm{I}_{r}$ and apply Proposition 1.17. 


\section{The Temporal Ultraviolet Limit}

\subsubsection{The Main Ingredients - Perturbation Theory}

Lemma 1.18 has given us a functional integral representation for the partition function, but has not told us very much about what the integrand looks like. The next step is to exploit the fact that $\frac{\beta}{p}$ is very small when $p$ is large to help us understand what $\left\langle\alpha_{\tau-\frac{\beta}{p}}\left|e^{-\frac{\beta}{p}(H-\mu N)}\right| \alpha_{\tau}\right\rangle$ looks like.

Proposition 1.19 There are constants $C, c$ such that the following holds. For each $\varepsilon>0$, there is an analytic function $F\left(\varepsilon, \alpha^{*}, \beta\right)$ such that

$$
\left\langle\alpha\left|e^{-\varepsilon(H-\mu N)}\right| \beta\right\rangle=e^{F\left(\varepsilon, \alpha^{*}, \beta\right)}
$$

on the domain $\|\alpha\|_{\infty},\|\beta\|_{\infty}<C \frac{1}{\sqrt{\varepsilon}}$, where, as usual $\|\alpha\|_{\infty}=\max _{\mathbf{x} \in X}|\alpha(\mathbf{x})|$. Write

$$
F\left(\varepsilon, \alpha^{*}, \beta\right)=\int_{X} d \mathbf{x} \alpha(\mathbf{x})^{*} \beta(\mathbf{x})-\varepsilon K\left(\alpha^{*}, \beta\right)+\mathcal{F}_{0}\left(\varepsilon, \alpha^{*}, \beta\right)
$$

where $K\left(\alpha^{*}, \beta\right)$ was defined in (1.3). Then

$$
\left|\mathcal{F}_{0}\left(\varepsilon, \alpha^{*}, \beta\right)\right| \leq c \varepsilon^{2}\left(\Phi^{2}+\|v\|_{1, \infty}^{2} \Phi^{6}\right)
$$

for all $0<\varepsilon \leq 1$ and $\|\alpha\|_{\infty},\|\beta\|_{\infty} \leq \Phi \leq \frac{1}{2} C \frac{1}{\sqrt{\varepsilon}}$.

Idea of Proof $\left\langle\alpha\left|e^{-\varepsilon(H-\mu N)}\right| \beta\right\rangle$ is an entire function of $\alpha^{*}$ and $\beta$ and a $C^{\infty}$ function of $\varepsilon$ for $\varepsilon \geq 0$. (Just plug in the definitions (1.41) of $|\alpha\rangle$ and $|\beta\rangle$ in terms of the standard basis and use that the operator norm of $e^{-\varepsilon(H-\mu N)}(H-\mu N)^{m}$ restricted to $\mathcal{F}_{n}$ is bounded by a constant times $(n+1)^{m}$ for all integers $m, n \geq 0$ and $\varepsilon \geq 0$.) But $\left\langle\alpha\left|e^{-\varepsilon(H-\mu N)}\right| \beta\right\rangle$ can take the value zero (see (Balaban et al., 2008a, Example 3.12)), so its logarithm need not be everywhere defined. Since $\langle\alpha \mid \beta\rangle=$ $e^{\int \alpha^{*}(\mathbf{x}) \beta(\mathbf{x}) d \mathbf{x}} \neq 0$, continuity implies that the matrix element has the representation

$$
\left\langle\alpha\left|e^{-\varepsilon(H-\mu N)}\right| \beta\right\rangle=e^{F\left(\varepsilon, \alpha^{*}, \beta\right)}
$$

in some neighbourhood of 0 , with $F\left(\varepsilon, \alpha^{*}, \beta\right)$ is analytic in $\alpha^{*}, \beta$. But is the neighbourhood big enough and what can we say about $F$ ? To go further, differentiate (1.45) with respect to $\varepsilon$ to give

$$
e^{F\left(\varepsilon, \alpha^{*}, \beta\right)} \frac{\partial F}{\partial \varepsilon}\left(\varepsilon, \alpha^{*}, \beta\right)=-\left\langle\alpha\left|(H-\mu N) e^{-\varepsilon(H-\mu N)}\right| \beta\right\rangle
$$

Now, downstairs on the right hand side, substitute in the definitions of $H$ and $N$ in terms of the annihilation and creation operators $\psi(\mathbf{x})$ and $\psi^{\dagger}(\mathbf{x})$ (see (1.38)) and use

$$
\psi(\mathbf{x})|\alpha\rangle=\alpha(\mathbf{x})|\alpha\rangle \quad \psi^{\dagger}(\mathbf{x})|\alpha\rangle=\frac{\partial}{\partial \alpha(\mathbf{x})}|\alpha\rangle
$$

This gives us the differential equation

$$
\frac{\partial}{\partial \varepsilon} F=-\mathcal{K}\left(\alpha^{*}, \frac{\partial}{\partial \alpha^{*}}\right) F-\iint_{X} d \mathbf{x} d \mathbf{y} \alpha(\mathbf{x})^{*} \alpha(\mathbf{y})^{*} v(\mathbf{x}, \mathbf{y}) \frac{\partial F}{\partial \alpha(\mathbf{x})^{*}} \frac{\partial F}{\partial \alpha(\mathbf{y})^{*}}
$$


where

$$
\begin{array}{rl}
\mathcal{K}\left(\alpha^{*}, \frac{\partial}{\partial \alpha^{*}}\right)=\iint_{X} d \mathbf{x} d \mathbf{y} \alpha(\mathbf{x})^{*} & h(\mathbf{x}, \mathbf{y}) \frac{\partial}{\partial \alpha(\mathbf{y})^{*}}-\mu \int_{X} d \mathbf{x} \alpha(\mathbf{x})^{*} \frac{\partial}{\partial \alpha(\mathbf{x})^{*}} \\
& +\iint_{X} d \mathbf{x} d \mathbf{y} \alpha(\mathbf{x})^{*} \alpha(\mathbf{y})^{*} v(\mathbf{x}, \mathbf{y}) \frac{\partial}{\partial \alpha(\mathbf{x})^{*}} \frac{\partial}{\partial \alpha(\mathbf{y})^{*}}
\end{array}
$$

The details are in (Balaban et al., 2008a, Lemma 3.8)). As $F$ also satisfies the initial condition

$$
F\left(0, \alpha^{*}, \beta\right)=\ln \langle\alpha \mid \beta\rangle=\int_{X} d \mathbf{x} \alpha(\mathbf{x})^{*} \beta(\mathbf{x})
$$

we now have a first order initial value problem for $F$, viewed as a function of $\varepsilon$. It is tedious but straight forward to convert this into a system of integral equations for coefficients in the Taylor expansion of $F\left(\varepsilon, \alpha^{*}, \beta\right)$ in powers of $\alpha^{*}$ and $\beta$. (See (Balaban et al., 2008a, Lemma 3.9).) The system can be solved and bounded by iteration. The details are in (Balaban et al., 2008a, Lemmas 3.8 and 3.9 and Proposition 3.6).

\subsubsection{Finishing off the Proof of Theorem 1.14}

So we now have

$$
\begin{aligned}
\operatorname{Tr} e^{-\beta(H-\mu N)}=\lim _{p \rightarrow \infty} \int d \mu_{p, \mathrm{R}(p)}\left(\alpha^{*}, \alpha\right) & \prod_{\tau \in \mathcal{T}_{p}} e^{-\int d \mathbf{y}\left[\alpha_{\tau}^{*}(\mathbf{y})-\alpha_{\tau-\varepsilon_{p}}^{*}(\mathbf{y})\right] \alpha_{\tau}(\mathbf{y})} e^{-\varepsilon_{p} K\left(\alpha_{\tau-\varepsilon_{p}}^{*}, \alpha_{\tau}\right)} \\
& \prod_{\tau \in \mathcal{T}_{p}} e^{-\mathcal{F}_{0}\left(\varepsilon_{p}, \alpha_{\tau-\varepsilon_{p}}^{*}, \alpha_{\tau}\right)}
\end{aligned}
$$

and we just have to show that discarding the $\mathcal{F}_{0}$ 's does not affect the value of the $p \rightarrow \infty$ limit. Before I outline the argument that this is the case, I'll make two remarks;

- If the functional integral representation is going to be used as a starting point for a renormalization group construction, it may not be necessary to show that discarding the $\mathcal{F}_{0}$ 's does not affect the value of the $p \rightarrow \infty$ limit. The bound on $\mathcal{F}_{0}$ provided by Proposition 1.19 may be adequate in itself.

- Observe that the sum

$$
\sum_{\tau \in \mathcal{T}_{p}} \mathcal{F}_{0}\left(\varepsilon_{p}, \alpha_{\tau-\varepsilon_{p}}^{*}, \alpha_{\tau}\right)
$$

has $p$ terms and that each term is of order $\varepsilon^{2}=\frac{1}{p^{2}}$. So the sum is of order $\frac{1}{p}$. This is a first hint that these terms disappear in the limit $p \rightarrow \infty$. But it does not prove anything, since the volume of the domain of integration is growing like $\mathrm{R}(p)^{2|X| p}$. (The order $\varepsilon^{2}$ in the bound of Proposition 1.19 is also multiplied by a $\Phi^{6} \leq \mathrm{R}(p)^{6}$, but it grows relatively slowly with $p$.)

Now here is an outline of the argument that we may discard the $\mathcal{F}_{0}$ 's. The details are in (Balaban et al., 2008a). Let $r>0$. Define, for $\mathcal{I}: \mathbb{C}^{2|X|} \rightarrow \mathbb{C}$, the seminorm

$$
\|\mathcal{I}\|_{r}=\sup _{\substack{\alpha, \phi \in \mathbb{C}^{X} \\|\alpha|_{X},|\phi|_{X} \leq r}}|\mathcal{I}(\alpha, \phi)|
$$




\section{The Temporal Ultraviolet Limit}

and, for $\mathcal{I}, \mathcal{J}: \mathbb{C}^{2|X|} \rightarrow \mathbb{C}$, with $\|\mathcal{I}\|_{r},\|\mathcal{J}\|_{r}<\infty$, the "r-product" of $\mathcal{I}, \mathcal{J}$

$$
\left(\mathcal{I} *_{r} \mathcal{J}\right)(\alpha, \gamma)=\int \mathcal{I}(\alpha, \phi) \mathcal{J}(\phi, \gamma) d \mu_{r}\left(\phi^{*}, \phi\right)
$$

where

$$
d \mu_{r}\left(\phi^{*}, \phi\right)=\prod_{\mathbf{x} \in X}\left[\frac{d \phi^{*}(\mathbf{x}) \wedge d \phi(\mathbf{x})}{2 \pi \imath} \chi(|\phi(\mathbf{x})|<r)\right]
$$

The $q^{\text {th }}$ power with respect to this product is denoted

$$
\mathcal{I}^{*_{r} q}=\overbrace{\mathcal{I} *_{r} \mathcal{I} *_{r} \cdots *_{r} \mathcal{I}}^{q \text { factors }}
$$

For each $\varepsilon>0$, set

$$
\begin{aligned}
\mathcal{I}_{\varepsilon}(\alpha, \phi) & =e^{-\frac{1}{2}\|\alpha\|^{2}-\frac{1}{2}\|\phi\|^{2}} e^{F\left(\varepsilon, \alpha^{*}, \phi\right)}=e^{-\frac{1}{2}\|\alpha\|^{2}-\frac{1}{2}\|\phi\|^{2}}\left\langle\alpha\left|e^{-\varepsilon(H-\mu N)}\right| \phi\right\rangle \\
\tilde{\mathcal{I}}_{\varepsilon}(\alpha, \phi) & =e^{-\frac{1}{2}\|\alpha\|^{2}-\frac{1}{2}\|\phi\|^{2}} e^{F\left(\varepsilon, \alpha^{*}, \phi\right)-\mathcal{F}_{0}\left(\varepsilon, \alpha^{*}, \phi\right)} \\
& =\exp \left\{-\frac{1}{2}\|\alpha\|^{2}-\frac{1}{2}\|\phi\|^{2}+\int d \mathbf{x} \alpha^{*}(\mathbf{x}) \phi(\mathbf{x})-\varepsilon K\left(\alpha^{*}, \phi\right)\right\}
\end{aligned}
$$

Lemma 1.18 and Proposition 1.19 state that, for $\mathrm{R}(p)$ obeying $\lim _{p \rightarrow \infty} p e^{-\frac{1}{2} \mathrm{R}(p)^{2}}=0$,

$$
\operatorname{Tr} e^{-\beta K}=\left.\lim _{p \rightarrow \infty} \int d \mu_{r}\left(\phi^{*}, \phi\right) \mathcal{I}_{\varepsilon}^{*_{r} p}(\phi, \phi)\right|_{\substack{r=\mathrm{R}(p) \\ \varepsilon=\beta / p}}
$$

and we would like to have

$$
\operatorname{Tr} e^{-\beta K}=\left.\lim _{p \rightarrow \infty} \int d \mu_{r}\left(\phi^{*}, \phi\right) \tilde{\mathcal{I}}_{\varepsilon}^{*_{r} p}(\phi, \phi)\right|_{\substack{r=\mathrm{R}(p) \\ \varepsilon=\beta / p}}
$$

instead. By Lemma 1.13, the operator $H-\mu N$ is bounded below. Say $H-\mu N \geq-K_{0} 1$. Then, for any $q \in \mathbb{N}$,

$$
\mathcal{I}_{\varepsilon}^{*_{r} q}(\alpha, \phi)=e^{-\frac{1}{2}\|\alpha\|^{2}-\frac{1}{2}\|\phi\|^{2}}\left\langle\alpha\left|\left(e^{-\varepsilon K} \mathrm{I}_{r}\right)^{q-1} e^{-\varepsilon K}\right| \phi\right\rangle
$$

implies that

$$
\left\|\mathcal{I}_{\varepsilon}^{*_{r} q}\right\|_{r} \leq e^{-\frac{1}{2}\|\alpha\|^{2}-\frac{1}{2}\|\phi\|^{2}}\|\alpha\|\left(e^{\varepsilon K_{0}}\left\|\mathrm{I}_{r}\right\|\right)^{q-1} e^{\varepsilon K_{0}}\|\phi\|=e^{q \varepsilon K_{0}}\left\|\mathrm{I}_{r}\right\|^{q-1} \leq e^{q \varepsilon K_{0}}
$$

for all $r>0$, by part (c) of Theorem 1.15. The difference

$$
\begin{aligned}
\left\|\mathcal{I}_{\varepsilon}-\tilde{\mathcal{I}}_{\varepsilon}\right\|_{r} & =\sup _{|\alpha|_{X},|\phi|_{X} \leq r}\left|e^{-\frac{1}{2}\|\alpha\|^{2}-\frac{1}{2}\|\phi\|^{2}}\left\langle\alpha\left|e^{-\varepsilon K}\right| \phi\right\rangle\left[1-e^{-\mathcal{F}_{0}\left(\varepsilon, \alpha^{*}, \phi\right)}\right]\right| \\
& \leq e^{\varepsilon K_{0}} \sup _{|\alpha|_{X},|\phi|_{X} \leq r}\left|\left[1-e^{-\mathcal{F}_{0}\left(\varepsilon, \alpha^{*}, \phi\right)}\right]\right| \\
& \leq e^{\varepsilon K_{0}} \text { const } \varepsilon^{2} r^{6}|X| e^{\operatorname{const}^{2} r^{6}|X|}
\end{aligned}
$$

by Proposition 1.19 (assuming that $1 \leq r \leq \frac{\text { const }}{\sqrt{\varepsilon}}$ ). The following proposition is, naturally, proven by induction in (Balaban et al., 2008a, Proposition 3.16). 
Proposition 1.20 Let $K_{0}, \varepsilon, \zeta>0$ and $0<\kappa<1$ and $r, C_{\beta} \geq 1$ obey

$$
C_{\beta}\left(\pi r^{2}\right)^{3|X|} \zeta^{1-\kappa} \leq \varepsilon
$$

Let $\mathcal{I}, \tilde{\mathcal{I}}: \mathbb{C}^{2|X|} \rightarrow \mathbb{C}$ obey

$$
\|\mathcal{I}-\tilde{\mathcal{I}}\|_{r} \leq \zeta \quad\left\|\mathcal{I}^{*_{r} q}\right\|_{r} \leq e^{q \varepsilon K_{0}} \text { for all } q \in \mathbb{N}
$$

Then, for all $q \in \mathbb{N}$ with $q \leq \frac{C_{\beta}}{\varepsilon}$,

$$
\begin{aligned}
\left\|\tilde{\mathcal{I}}^{*_{r} q}\right\|_{r} & \leq e^{q \varepsilon\left(K_{0}+\zeta^{\kappa}\right)} \\
\left\|\tilde{\mathcal{I}}^{*_{r} q}-\mathcal{I}^{*_{r} q}\right\|_{r} & \leq \zeta^{\kappa} e^{q \varepsilon\left(K_{0}+\zeta^{\kappa}\right)} \\
\int d \mu_{r}\left(\phi^{*}, \phi\right)\left|\tilde{\mathcal{I}}^{*_{r} q}(\phi, \phi)-\mathcal{I}^{*_{r} q}(\phi, \phi)\right| & \leq \zeta^{\kappa} e^{q \varepsilon\left(K_{0}+\zeta^{\kappa}\right)}
\end{aligned}
$$

It now suffices to apply Proposition 1.20 with $\zeta=\varepsilon^{3 / 2}, r=\mathrm{R}(p), p=\frac{\beta}{\varepsilon}, \kappa=\frac{1}{12}$ and $C_{\beta}=\beta$. Since

$C_{\beta}\left(\pi r^{2}\right)^{3|X|} \zeta^{1-\kappa}=\beta\left(\pi \mathrm{R}\left(\frac{\beta}{\varepsilon}\right)^{2}\right)^{3|X|} \varepsilon^{\frac{33}{24}} \leq \varepsilon \quad$ if $\mathrm{R}(p)<p^{\frac{1}{24|X|}}$ and $\varepsilon$ is small enough $e^{\varepsilon K_{0}} \operatorname{const}^{2} R\left(\frac{\beta}{\varepsilon}\right)^{6}|X| e^{\operatorname{const}^{2} R\left(\frac{\beta}{\varepsilon}\right)^{6}|X|} \leq \varepsilon^{\frac{3}{2}} \quad$ if $\mathrm{R}(p)<p^{\frac{1}{24}}$ and $\varepsilon$ is small enough

the hypotheses of Proposition 1.20 are satisfied.

\subsubsection{Cylinder Set Measures}

There are other rigorous functional integral representations used in quantum mechanics, quantum field theory and condensed matter physics. Probably the most elegant and powerful class of such representations use cylinder set measures. Cylinder set measures refer to measures on infinite dimensional vector spaces that are built by taking a limit of a collection of probability measures defined on finite dimensional subspaces of the vector space. The measures on the different subspaces have to be consistent with each other in a natural sense that I will now explain. Let $\mathcal{I}$ be a countable set and take as our vectors space

$$
\mathbb{R}^{\mathcal{I}}=\left\{\vec{x}=\left(x_{i}\right)_{i \in \mathcal{I}} \mid x_{i} \in \mathbb{R} \text { for all } i \in \mathcal{I}\right\}
$$

For each finite subset $I \subset \mathcal{I}$ define the subspace $\mathbb{R}^{I}$ of $\mathbb{R}^{\mathcal{I}}$ by

$$
\mathbb{R}^{I}=\left\{\left(x_{i}\right)_{i \in \mathcal{I}} \in \mathbb{R}^{\mathcal{I}} \mid x_{i}=0 \text { for all } i \in \mathcal{I} \backslash I\right\}
$$

and define the natural projection $P_{I}: \mathbb{R}^{\mathcal{I}} \rightarrow \mathbb{R}^{I}$ by

$$
\left(P_{I} \vec{x}\right)_{j}= \begin{cases}x_{j} & \text { if } j \in I \\ 0 & \text { if } j \in \mathcal{I} \backslash I\end{cases}
$$


Suppose that we are given, for each finite $I \subset \mathcal{I}$, a probability measure $\mu_{I}$ on $\mathbb{R}^{I}$. This family of measures is said to be consistent if for each pair of finite subsets $I, I^{\prime} \subset \mathcal{I}$ obeying $I \subset I^{\prime}$ and for each measurable $A \subset \mathbb{R}^{I}$, we have

$$
\mu_{I^{\prime}}\left(\left\{\vec{x} \in \mathbb{R}^{I^{\prime}} \mid P_{I} \vec{x} \in A\right\}\right)=\mu_{I}(A)
$$

The theorem that "takes the limit" is

Theorem 1.21 (Kolmogorov's Theorem) Let $\mathcal{I}$ be a countable set and let a probability measure $\mu_{I}$ on $\mathbb{R}^{I}$ be given for each finite set $I \subset \mathcal{I}$ so that the family of $\mu_{I}$ 's are consistent. Then there are a probability measure space $(X, \mathcal{F}, \mu)$ and random variables $\left\{f_{\alpha}\right\}_{\alpha \in \mathcal{I}}$ so that $\mu_{I}$ is the joint probability distribution of $\left\{f_{\alpha}\right\}_{\alpha \in I}$. That is

$$
\mu_{I}(A)=\mu\left(\left\{x \in X \mid P_{I}\left(f_{\alpha}(x)\right)_{\alpha \in \mathcal{I}} \in A\right\}\right)
$$

for all measurable $A \subset \mathbb{R}^{I}$. Moreover this space is unique in the sense that if $\left(X^{\prime}, \mathcal{F}^{\prime}, \mu^{\prime}\right)$ and $\left\{f_{\alpha}^{\prime}\right\}_{\alpha \in \mathcal{I}}$ also have these properties and if $\mathcal{F}$ (and respectively, $\mathcal{F}^{\prime}$ ) is the smallest $\sigma$-field which respect to which the $f_{\alpha}$ (respectively $f_{\alpha}^{\prime}$ ) are measurable, then there is an isomorphism of the probability measure spaces under which each $f_{\alpha}$ corresponds to $f_{\alpha}^{\prime}$.

A very convenient tool for constructing cylinder measures is (Minlos, 1959)

Theorem 1.22 (Minlos' Theorem) A necessary and sufficient condition for a function $\Phi: \mathcal{S}\left(\mathbb{R}^{\nu}\right) \rightarrow \mathbb{C}$ to be the Fourier transform

$$
\Phi(\varphi)=\int e^{i T(\varphi)} d \mu(T)
$$

of a cylinder set probability measure $\mu$ on $\mathcal{S}^{\prime}\left(\mathbb{R}^{\nu}\right)$ is that $\Phi(0)=1$, $\Phi$ be positive definite and $\Phi$ be continuous in the Fréchet topology on $\mathcal{S}\left(\mathbb{R}^{\nu}\right)$.

Here

- $\mathcal{S}\left(\mathbb{R}^{\nu}\right)$ is Schwartz space, the space of all $C^{\infty}$ functions on $\mathbb{R}^{\nu}$ all of whose derivatives decay faster than any polynomial at infinity,

- $\mathcal{S}^{\prime}\left(\mathbb{R}^{\nu}\right)$ is the space of tempered distributions, the space of all continuous linear functions on $\mathcal{S}\left(\mathbb{R}^{\nu}\right)$

- a cylinder set measure on $\mathcal{S}^{\prime}\left(\mathbb{R}^{\nu}\right)$ is a measure on the $\sigma$-field generated by the functions $\left\{T \mapsto T(\varphi) \mid \varphi \in \mathcal{S}\left(\mathbb{R}^{\nu}\right)\right\}$

- $\Phi: \mathcal{S}\left(\mathbb{R}^{\nu}\right) \rightarrow \mathbb{C}$ is positive definite if $\sum_{i, j=1}^{n} \overline{z_{i}} z_{j} \Phi\left(\varphi_{i}-\varphi_{j}\right) \geq 0$ for all $n \in \mathbb{N}$, $z_{1}, \cdots, z_{n} \in \mathbb{C}$ and $\varphi_{1}, \cdots, \varphi_{n} \in \mathcal{S}\left(\mathbb{R}^{n}\right)$

For an expositions on cylinder set measures, see (Gel'fand and Vilenkin, 1968; Simon, 2005). For applications of cylinder set measures to Brownian motion and Wiener processes, see (Nelson, 1964, Appendix A) and (Durrett, 2010). For applications of cylinder set measures to Schrödinger operators, see (Simon, 2005). For applications of cylinder set measures to field theory and statistical mechanics, see (Ginibre, 1971; Fröhlich, 1974; Feldman and Osterwalder, 1976; Glimm and Jaffe, 1987). 


\subsubsection{A Warning About Complex Measures}

It is critical that cylinder measures are (real-valued) probability measures. The point is that complex measures must have finite total mass. (When you compute the measure of a complicated set by cutting it up into countable many disjoint sets and adding up the measures of the pieces, it is important that it not matter what order you do the sum in. And that is the case only if the sum is absolutely convergent.) That dramatically limits the class of complex measures on infinite dimensional vector spaces. In particular, in our case, the exponent $\mathcal{A}\left(\alpha^{*}, \alpha\right)$ is complex and as a result, $e^{\mathcal{A}\left(\alpha^{*}, \alpha\right)}$ oscillates wildly. In contrast to Wiener measure, $\frac{1}{\text { const }} \prod \frac{d \alpha_{\tau}^{*}(\mathbf{x}) \wedge d \alpha_{\tau}(\mathbf{x})}{2 \pi i} e^{\text {part of } \mathcal{A}\left(\alpha^{*}, \alpha\right)}$ cannot be turned into an ordinary well-defined complex measure on some space of paths.

Here is a well-known example, due to Cameron (Cameron, 1960; Cameron, 1963), that illustrates the phenomenon. Let $\left[C_{i j}\right]_{i, j \in \mathbb{N}}$ be a "matrix" with infinitely many rows and columns. Assume that $C$ has real entries, is symmetric and is strictly positive definite in the sense that $\sum_{i, j} \alpha_{i} C_{i, j} \alpha_{j}>0$ for all nonzero, real "vectors" $\left[\alpha_{i}\right]_{i \in \mathbb{N}}$ having only finitely many nonzero components. A simple example of such a matrix is the identity matrix

$$
\delta_{i, j}= \begin{cases}1 & \text { if } i=j \\ 0 & \text { if } i \neq j\end{cases}
$$

Another example is $-\Delta_{i, j}+m^{2} \delta_{i, j}$ where $\Delta_{i, j}$ is the discrete Laplacian on $\mathbb{Z}^{3}$ and $i, j$ refers to some arbitrary ordering of the points in $\mathbb{Z}^{3}$. Fix some $\sigma \in \mathbb{C}$ with $\operatorname{Re} \sigma>0$. Consider, for each $n \in \mathbb{N}$, the measure

$$
d \mu_{n}(\vec{\alpha})=\frac{e^{-\frac{1}{2} \sigma \vec{\alpha} \cdot C \vec{\alpha}} d^{n} \vec{\alpha}}{\int_{\mathbb{R}^{n}} e^{-\frac{1}{2} \sigma \vec{\alpha} \cdot C \vec{\alpha}} d^{n} \vec{\alpha}}
$$

on $\mathbb{R}^{n}$. Here, in computing $\vec{\alpha} \cdot C \vec{\alpha}$, set $\alpha_{j}=0$ for all $j>n$. If $\sigma$ is real, this is a legitimate probability measure. If, in addition, $C$ is diagonal it is trivial to apply Kolmogorov's Theorem and create a cylinder set measure. (For many other $C$ 's you can also create a cylinder set measures, with more work.) If $\operatorname{Im} \sigma \neq 0, \mu_{n}$ is still a legitimate (complex) measure on $\mathbb{R}^{n}$ and is still normalized so that $\int_{\mathbb{R}^{n}} d \mu_{n}(\vec{\alpha})=1$.

In particular, if we write $C_{n}=\left[C_{i j}\right]_{1 \leq i, j \leq n}$, then, by making an orthogonal change of variables so as to diagonalize $C_{n}$, it is easy to see that

$$
\int_{\mathbb{R}^{n}} e^{-\frac{1}{2} \sigma \vec{\alpha} \cdot C \vec{\alpha}} d^{n} \vec{\alpha}=\left[\left(\frac{\pi}{\sigma}\right)^{n} \frac{1}{\operatorname{det} C_{n}}\right]^{1 / 2} \neq 0
$$

(We aren't going to care which square root is used.) The total mass of $\mu_{n}$ is

$$
\int_{\mathbb{R}^{n}}\left|d \mu_{n}(\vec{\alpha})\right|=\frac{\int_{\mathbb{R}^{n}}\left|e^{-\frac{1}{2}(\sigma \vec{\alpha} \cdot C \vec{\alpha}}\right| d^{n} \vec{\alpha}}{\left|\int_{\mathbb{R}^{n}} e^{-\frac{1}{2} \sigma \vec{\alpha} \cdot C \vec{\alpha}} d^{n} \vec{\alpha}\right|}=\frac{\int_{\mathbb{R}^{n}} e^{-\frac{1}{2}(\operatorname{Re} \sigma) \vec{\alpha} \cdot C \vec{\alpha}} d^{n} \vec{\alpha}}{\left|\int_{\mathbb{R}^{n}} e^{-\frac{1}{2} \sigma \vec{\alpha} \cdot C \vec{\alpha}} d^{n} \vec{\alpha}\right|}=\left\{\frac{|\sigma|}{\operatorname{Re} \sigma}\right\}^{n / 2}
$$

Since $\operatorname{Im} \sigma \neq 0$, this tends to infinity as $n \rightarrow \infty$ and we can't get a legitimate complex measure in the limit $n \rightarrow \infty$. Another model computation of this type which is closer to the integral of Theorem 1.14 is given in (Balaban et al., 2008a, Appendix A). 


\subsubsection{Grassmann Integrals}

Another class of functional integrals are Grassmann integrals. They are often used in fermionic models. Grassmann integrals are certain linear functionals defined on Grassmann algebras, which are a particularly simple class of algebras. The reason that the linear functionals are called "integrals" is that they have, up to signs, all of the usual algebraic properties of integrals, including, for example, integration by parts. For discussions of Grassmann integrals, see (Berezin, 1966; Feldman et al., 2002) and (Salmhofer, 1999, Appendix B).

\subsection{A Simple High Temperature Expansion}

High temperature expansions are extremely widely used tools in rigorous treatments of quantum field theories and statistical mechanical systems (and not just at high temperatures). This section is concerned with a very simple example of such an expansion. There are many other high temperature expansions. At the end of this section, I'll mention some others and give some references.

\subsubsection{Motivation - A Renormalization Group Construction Protocol}

Here is a cartoon description of a commonly used procedure for constructing and analyzing quantum field theories and models in condensed matter physics and statistical mechanics.

- Express the quantities of interest as functional integrals like

$$
\mathcal{G}(\Psi)=\ln \frac{\int e^{\mathcal{A}(\Psi, \Phi)} d \mu(\Phi)}{\int e^{\mathcal{A}(0, \Phi)} d \mu(\Phi)}
$$

- Factor the measure $d \mu(\Phi)=\prod_{\ell=1}^{\infty} d \mu_{\ell}\left(\varphi_{\ell}\right)$ to express

$$
\mathcal{G}(\Psi)=\ln \frac{\int e^{\mathcal{A}\left(\Psi, \varphi_{1}, \varphi_{2}, \cdots\right)} \prod_{\ell=1}^{\infty} d \mu_{\ell}\left(\varphi_{\ell}\right)}{\int e^{\mathcal{A}\left(0, \varphi_{1}, \varphi_{2}, \cdots\right)} \prod_{\ell=1}^{\infty} d \mu_{\ell}\left(\varphi_{\ell}\right)}
$$

- Do the integrals one at a time. Define the "effective action at scale $n$ " by

$$
\mathcal{A}_{n}\left(\Psi, \varphi_{n+1}, \varphi_{n+2}, \cdots\right)=\ln \frac{\int e^{\mathcal{A}\left(\Psi, \varphi_{1}, \varphi_{2}, \cdots\right)} \prod_{\ell=1}^{n} d \mu_{\ell}\left(\varphi_{\ell}\right)}{\int e^{\mathcal{A}\left(0, \varphi_{1}, \cdots, \varphi_{n}, 0, \cdots\right)} \prod_{\ell=1}^{n} d \mu_{\ell}\left(\varphi_{\ell}\right)}
$$

Then

$$
\mathcal{A}_{n}(\psi)=\ln \frac{\int e^{\mathcal{A}_{n-1}(\psi, \varphi)} d \mu_{n}(\varphi)}{\int e^{\mathcal{A}_{n-1}(0, \varphi)} d \mu_{n}(\varphi)}
$$

where $\varphi=\varphi_{n}$ and $\psi=\left(\Psi, \varphi_{n+1}, \varphi_{n+2}, \cdots\right)$.

The decimation procedure of Sections 1.2 and 1.3 was like this. To be able to implement such a procedure, you have to be able to prove bounds on integrals like in (1.46). In this section, we'll derive such bounds. 


\subsubsection{The Main Theorem}

Let $X(=$ space $)$ be a finite set. Let $d \mu_{0}(z)$ be a normalized measure on $\mathbb{C}$ that is supported in $|z| \leq r$ for some constant $r$. We endow $\mathbb{C}^{X}$ with the ultralocal product measure

$$
d \mu(\varphi)=\prod_{\mathbf{x} \in X} d \mu_{0}(\varphi(\mathbf{x}))
$$

Theorem Let $w$ and $W$ be weight systems for 1 and 2 fields, respectively, that obey

$$
W(\overrightarrow{\mathbf{x}}, \overrightarrow{\mathbf{y}}) \geq(4 r)^{n(\overrightarrow{\mathbf{y}})} w(\overrightarrow{\mathbf{x}})
$$

Let $F: \mathbb{C}^{|X|} \times \mathbb{C}^{|X|} \rightarrow \mathbb{C}$ be analytic on a neighbourhood of the origin. If $F(\psi, \varphi)$ obeys $\|F\|_{W}<\frac{1}{16}$, then there is an analytic function $f(\psi)$ such that

$$
\frac{\int e^{F(\psi, \varphi)} d \mu(\varphi)}{\int e^{F(0, \varphi)} d \mu(\varphi)}=e^{f(\psi)} \quad \text { and } \quad\|f\|_{w} \leq \frac{\|F\|_{W}}{1-16\|F\|_{W}}
$$

(I'll fill in the missing definitions later and then restate the Theorem and call it Theorem 1.29.)

\subsubsection{Outline of the Proof - Algebra}

We'll first do some algebra and end up with an explicit (but messy) formula for $f(\psi)$ in terms of $F(\psi, \varphi)$. After that we'll introduce the norms and do the bounds which show that the formula makes sense and that the Theorem is true. We use the notation

$$
\begin{aligned}
& \mathbf{x} \in X=\text { space, a finite set } \\
& \overrightarrow{\mathbf{x}} \in \mathcal{X}=\text { multispace }=\bigcup_{n \geq 0} X^{n}=\left\{\left(\mathbf{x}_{1}, \cdots, \mathbf{x}_{n}\right) \in X^{n} \mid n \geq 0\right\}
\end{aligned}
$$

and, for $\overrightarrow{\mathbf{x}}=\left(\mathbf{x}_{1}, \cdots, \mathbf{x}_{n}\right) \in X^{n}, \overrightarrow{\mathbf{y}}=\left(\mathbf{y}_{1}, \cdots, \mathbf{y}_{m}\right) \in X^{m}$ and $\varphi: X \rightarrow \mathbb{C}$,

$$
\begin{aligned}
n(\overrightarrow{\mathbf{x}}) & =n \\
\overrightarrow{\mathbf{x}} \circ \overrightarrow{\mathbf{y}} & =\left(\mathbf{x}_{1}, \cdots, \mathbf{x}_{n}, \mathbf{y}_{1}, \cdots, \mathbf{y}_{m}\right) \in X^{n+m} \\
\varphi(\overrightarrow{\mathbf{x}}) & =\varphi\left(\mathbf{x}_{1}\right) \varphi\left(\mathbf{x}_{2}\right) \cdots \varphi\left(\mathbf{x}_{n}\right) \\
\operatorname{supp}(\overrightarrow{\mathbf{x}}) & =\left\{\mathbf{x}_{1}, \cdots, \mathbf{x}_{n}\right\} \subset X
\end{aligned}
$$

By hypothesis, $F: \mathbb{C}^{|X|} \times \mathbb{C}^{|X|} \rightarrow \mathbb{C}$ is analytic on a neighbourhood of the origin. We shall end up showing that $f: \mathbb{C}^{|X|} \rightarrow \mathbb{C}$ is analytic too. So there are unique expansions

$$
F(\psi, \varphi)=\sum_{\overrightarrow{\mathbf{x}}, \overrightarrow{\mathbf{y}} \in \mathcal{X}} A(\overrightarrow{\mathbf{x}}, \overrightarrow{\mathbf{y}}) \psi(\overrightarrow{\mathbf{x}}) \varphi(\overrightarrow{\mathbf{y}}) \quad f(\psi)=\sum_{\overrightarrow{\mathbf{x}} \in \mathcal{X}} a(\overrightarrow{\mathbf{x}}) \psi(\overrightarrow{\mathbf{x}})
$$

with $A(\overrightarrow{\mathbf{x}}, \overrightarrow{\mathbf{y}}), a(\overrightarrow{\mathbf{x}})$ invariant under permutations of the components of $\overrightarrow{\mathbf{x}}$ and under permutations of the components of $\overrightarrow{\mathbf{y}}$. We are about to do an integral over $\varphi$. Hide the $\psi$ dependence of $F$ by setting 


$$
\alpha(\overrightarrow{\mathbf{y}})=\sum_{\overrightarrow{\mathbf{x}} \in \mathcal{X}} A(\overrightarrow{\mathbf{x}}, \overrightarrow{\mathbf{y}}) \psi(\overrightarrow{\mathbf{x}})
$$

With this notation

$$
F(\psi, \varphi)=\sum_{\overrightarrow{\mathbf{y}} \in \mathcal{X}} \alpha(\overrightarrow{\mathbf{y}}) \varphi(\overrightarrow{\mathbf{y}})
$$

By factoring $e^{F(\psi, 0)}$ out of the integral in the numerator of $(1.32)$ and $e^{F(0,0)}$ out of the integral in the denominator of $(1.32)$ and moving $F(\psi, 0)-F(0,0)$ into $f(\psi)$, we may assume that $F(\psi, 0)=0$. (Check yourself that the bound is preserved by this operation.) Expand the exponential to give

$$
e^{F(\psi, \varphi)}=\sum_{\ell=0}^{\infty} \frac{1}{\ell !} F(\psi, \varphi)^{\ell}=1+\sum_{\ell=1}^{\infty} \frac{1}{\ell !} \sum_{\overrightarrow{\mathbf{y}}_{1}, \cdots, \overrightarrow{\mathbf{y}}_{\ell} \in \mathcal{X}} \alpha\left(\overrightarrow{\mathbf{y}}_{1}\right) \cdots \alpha\left(\overrightarrow{\mathbf{y}}_{\ell}\right) \varphi\left(\overrightarrow{\mathbf{y}}_{1}\right) \cdots \varphi\left(\overrightarrow{\mathbf{y}}_{\ell}\right)
$$

The decay properties of the coefficients $a(\overrightarrow{\mathbf{x}})$ in (1.47) are extremely important. Those coefficients are going to built out of products like $A\left(\overrightarrow{\mathbf{x}}_{1}, \overrightarrow{\mathbf{y}}_{1}\right) \cdots A\left(\overrightarrow{\mathbf{x}}_{\ell}, \overrightarrow{\mathbf{y}}_{\ell}\right)$ with $\overrightarrow{\mathbf{x}}=\left(\overrightarrow{\mathbf{x}}_{1}, \cdots, \overrightarrow{\mathbf{x}}_{\ell}\right)$. We are told (it is built into the norm $\left.\|\cdot\|_{W}\right)$ that $A(\overrightarrow{\mathbf{x}}, \overrightarrow{\mathbf{y}})$ decays as the components of $(\overrightarrow{\mathbf{x}}, \overrightarrow{\mathbf{y}})$ are separated. But that does not in general imply that $A\left(\overrightarrow{\mathbf{x}}_{1}, \overrightarrow{\mathbf{y}}_{1}\right) \cdots A\left(\overrightarrow{\mathbf{x}}_{\ell}, \overrightarrow{\mathbf{y}}_{\ell}\right)$ decays as the components of $\overrightarrow{\mathbf{x}}=\left(\overrightarrow{\mathbf{x}}_{1}, \cdots, \overrightarrow{\mathbf{x}}_{\ell}\right)$ are separated. But if we know in addition, for example, that, for each $1 \leq j \leq \ell-1$, $\overrightarrow{\mathbf{y}}_{j}$ has a component that is equal to some component of $\overrightarrow{\mathbf{y}}_{j+1}$, then $A\left(\overrightarrow{\mathbf{x}}_{1}, \overrightarrow{\mathbf{y}}_{1}\right) \cdots A\left(\overrightarrow{\mathbf{x}}_{\ell}, \overrightarrow{\mathbf{y}}_{\ell}\right)$ does decay as the components of $\overrightarrow{\mathbf{x}}=\left(\overrightarrow{\mathbf{x}}_{1}, \cdots, \overrightarrow{\mathbf{x}}_{\ell}\right)$ are separated.

We now built some machinery to keep track of such component overlaps. Define the incidence graph $G\left(\overrightarrow{\mathbf{y}}_{1}, \cdots, \overrightarrow{\mathbf{y}}_{\ell}\right)$ to be the labelled graph with

- vertices $\{1, \cdots, \ell\}$ and

- an edge between $i \neq j$ when $\operatorname{supp} \overrightarrow{\mathbf{y}}_{i} \cap \operatorname{supp} \overrightarrow{\mathbf{y}}_{j} \neq \emptyset$.

For a subset of $Z \subset X$, denote by $\mathcal{C}(Z)$ the set of all ordered tuples $\left(\overrightarrow{\mathbf{y}}_{1}, \cdots, \overrightarrow{\mathbf{y}}_{n}\right)$ such that

- $Z=\operatorname{supp} \overrightarrow{\mathbf{y}}_{1} \cup \cdots \cup \operatorname{supp} \overrightarrow{\mathbf{y}}_{n}$.

- $G\left(\overrightarrow{\mathbf{y}}_{1}, \cdots, \overrightarrow{\mathbf{y}}_{n}\right)$ is connected.

We call such a tuple a connected cover of $Z$. Now reorganize the $\ell^{\text {th }}$ term of $(1.48)$ according to the supports of the connected components of $G\left(\overrightarrow{\mathbf{y}}_{1}, \cdots, \overrightarrow{\mathbf{y}}_{\ell}\right)$.

$$
\begin{aligned}
& \sum_{\overrightarrow{\mathbf{y}}_{1}, \cdots, \overrightarrow{\mathbf{y}}_{\ell} \in \mathcal{X}} \alpha\left(\overrightarrow{\mathbf{y}}_{1}\right) \cdots \alpha\left(\overrightarrow{\mathbf{y}}_{\ell}\right) \varphi\left(\overrightarrow{\mathbf{y}}_{1}\right) \cdots \varphi\left(\overrightarrow{\mathbf{y}}_{\ell}\right)
\end{aligned}
$$

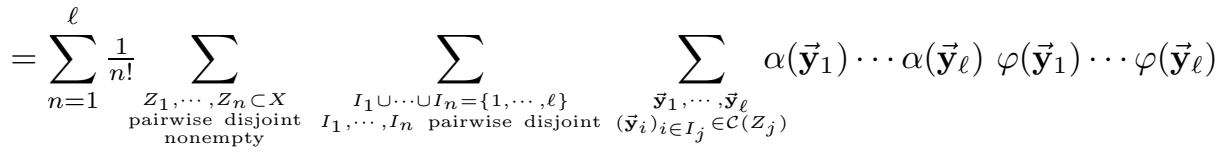

Fix, for the moment, pairwise disjoint nonempty subsets $Z_{1}, \cdots, Z_{n}$ of $X$ and $\ell \geq n$. Then 


$$
\begin{aligned}
& \sum_{\substack{I_{1} \cup \ldots \cup I_{n}=\{1, \ldots, \ell\} \\
I_{1}, \cdots, I_{n}}} \sum_{\substack{\overrightarrow{\mathbf{y}}_{1}, \cdots, \overrightarrow{\mathbf{y}}_{\ell} \\
\text { disjoint } \\
\left(\overrightarrow{\mathbf{y}}_{i}, i \in I_{j}\right) \in \mathcal{C}\left(Z_{j}\right)}} \alpha\left(\overrightarrow{\mathbf{y}}_{1}\right) \cdots \alpha\left(\overrightarrow{\mathbf{y}}_{\ell}\right) \varphi\left(\overrightarrow{\mathbf{y}}_{1}\right) \cdots \varphi\left(\overrightarrow{\mathbf{y}}_{\ell}\right) \\
& =\sum_{\substack{k_{1}, \cdots, k_{n} \geq 1 \\
k_{1}+\cdots+k_{n}=\ell}} \sum_{\substack{I_{1}, \cdots, I_{n} \subset\{1, \ldots, \ell\} \\
I_{1}, \cdots, I_{n} \text { disjoint } \\
\left|I_{j}\right|=k_{j}}} \sum_{\substack{\overrightarrow{\mathbf{y}}_{1}, \cdots, \overrightarrow{\mathbf{y}}_{\ell} \\
\left(\overrightarrow{\mathbf{y}}_{i}, i \in I_{j}\right) \in \mathcal{C}\left(Z_{j}\right)}} \alpha\left(\overrightarrow{\mathbf{y}}_{1}\right) \cdots \alpha\left(\overrightarrow{\mathbf{y}}_{\ell}\right) \varphi\left(\overrightarrow{\mathbf{y}}_{1}\right) \cdots \varphi\left(\overrightarrow{\mathbf{y}}_{\ell}\right) \\
& =\sum_{\substack{k_{1}, \cdots, k_{n} \geq 1 \\
k_{1}+\cdots+k_{n}=\ell}} \frac{\ell !}{k_{1} ! \cdots k_{n} !} \sum_{\substack{\left(\overrightarrow{\mathbf{y}}_{1}, \cdots, \overrightarrow{\mathbf{y}}_{k_{1}}\right) \in \mathcal{C}\left(Z_{1}\right) \\
\vdots}} \alpha\left(\overrightarrow{\mathbf{y}}_{1}\right) \cdots \alpha\left(\overrightarrow{\mathbf{y}}_{\ell}\right) \varphi\left(\overrightarrow{\mathbf{y}}_{1}\right) \cdots \varphi\left(\overrightarrow{\mathbf{y}}_{\ell}\right)
\end{aligned}
$$

As the measure $\mu$ factorizes with each factor normalized, and the different $Z_{j}$ 's are disjoint,

$$
\int \varphi\left(\overrightarrow{\mathbf{y}}_{1}\right) \cdots \varphi\left(\overrightarrow{\mathbf{y}}_{\ell}\right) d \mu(\varphi)=\prod_{j=1}^{n} \int \varphi\left(\overrightarrow{\mathbf{y}}_{p_{j-1}+1}\right) \cdots \varphi\left(\overrightarrow{\mathbf{y}}_{p_{j}}\right) d \mu(\varphi)
$$

(where $p_{0}=0$ and, for $1 \leq j \leq n, p_{j}=k_{1}+\cdots+k_{j}$ ).

Substituting (1.50) into (1.49) and then (1.49) into (1.48) and then integrating and applying (1.51) gives

$$
\begin{aligned}
& \int e^{F(\psi, \varphi)} d \mu(\varphi)=1+\sum_{\ell=1}^{\infty} \frac{1}{\ell !} \sum_{n=1}^{\ell} \frac{1}{n !} \sum_{\substack{Z_{1}, \cdots, Z_{n} \subset X \\
\text { pairwise disjoint } \\
\text { nonempty }}} \sum_{\substack{k_{1}, \cdots, k_{n} \geq 1 \\
k_{1}+\cdots+k_{n}=\ell}} \frac{\ell !}{k_{1} ! \cdots k_{n} !} \cdots \\
& =1+\sum_{n=1}^{\infty} \sum_{\ell=n}^{\infty} \frac{1}{n !} \sum_{\substack{Z_{1}, \cdots, z_{n} \subset X \\
\text { pairwise disjoint } \\
\text { nonempty }}} \sum_{\substack{k_{1}, \cdots, k_{n} \geq 1 \\
k_{1}+\cdots+k_{n}=\ell}} \frac{1}{k_{1} ! \cdots k_{n} !} \cdots \\
& =1+\sum_{n=1}^{\infty} \frac{1}{n !} \sum_{\substack{Z_{1}, \cdots, Z_{n} \subset X \\
\text { pairwise disjoint } \\
\text { nonempty }}} \sum_{k_{1}, \cdots, k_{n} \geq 1} \frac{1}{k_{1} ! \cdots k_{n} !} \cdots
\end{aligned}
$$

so that

$$
\int e^{F(\psi, \varphi)} d \mu(\varphi)=1+\sum_{n=1}^{\infty} \frac{1}{n !} \sum_{\substack{Z_{1}, \cdots, Z_{n} \subset x \\ \text { pairwise disjoint }}} \prod_{j=1}^{n} \Phi\left(Z_{j}\right)
$$

where, for $\emptyset \neq Z \subset X$,

$$
\Phi(Z)=\sum_{k=1}^{\infty} \frac{1}{k !} \sum_{\left(\overrightarrow{\mathbf{y}}_{1}, \cdots, \overrightarrow{\mathbf{y}}_{k}\right) \in \mathcal{C}(Z)} \alpha\left(\overrightarrow{\mathbf{y}}_{1}\right) \cdots \alpha\left(\overrightarrow{\mathbf{y}}_{k}\right) \int \varphi\left(\overrightarrow{\mathbf{y}}_{1}\right) \cdots \varphi\left(\overrightarrow{\mathbf{y}}_{k}\right) d \mu(\varphi)
$$

and $\Phi(\emptyset)=0$.

We next rewrite (1.52) so that it looks like "the sum of the values of all Feynman diagrams". We do this so that we can use the standard fact that "the logarithm of the 


\section{The Temporal Ultraviolet Limit}

sum of the values of all Feynman diagrams is the sum of the values of all connected Feynman diagrams". If we define

$$
\zeta\left(Z, Z^{\prime}\right)= \begin{cases}0 & \text { if } Z \cap Z^{\prime} \neq \emptyset \\ 1 & \text { if } Z \text { and } Z^{\prime} \text { are disjoint }\end{cases}
$$

and $G_{n}=\left\{\{i, j\} \subset \mathbb{N}^{2} \mid 1 \leq i<j \leq n\right\}$ is the complete graph on $\{1, \cdots, n\}$, then

$$
\begin{aligned}
\int e^{F(\psi, \varphi)} d \mu(\varphi) & =1+\sum_{n=1}^{\infty} \frac{1}{n !} \sum_{Z_{1}, \cdots, Z_{n} \subset X} \prod_{\{i, j\} \in G_{n}} \zeta\left(Z_{i}, Z_{j}\right) \prod_{j=1}^{n} \Phi\left(Z_{j}\right) \\
& =1+\sum_{n=1}^{\infty} \frac{1}{n !} \sum_{Z_{1}, \cdots, Z_{n}}\left(\sum_{g \subset G_{n}} \prod_{\{i, j\} \in g}\left(\zeta\left(Z_{i}, Z_{j}\right)-1\right)\right) \prod_{j=1}^{n} \Phi\left(Z_{j}\right) \\
& =1+\sum_{n=1}^{\infty} \frac{1}{n !} \sum_{Z_{1}, \cdots, Z_{n} \subset X} \rho\left(Z_{1}, \cdots, Z_{n}\right) \prod_{j=1}^{n} \Phi\left(Z_{j}\right)
\end{aligned}
$$

where

$$
\rho\left(Z_{1}, \cdots, Z_{n}\right)= \begin{cases}1 & \text { if } n=1 \\ \sum_{g \subset G_{n}} \prod_{\{i, j\} \in g}\left(\zeta\left(Z_{i}, Z_{j}\right)-1\right) & \text { if } n \geq 2\end{cases}
$$

Define

$$
\rho^{T}\left(Z_{1}, \cdots, Z_{n}\right)=\left\{\begin{array}{lr}
1 & \text { if } n=1 \\
\sum_{g \in \mathcal{C}_{n}} \prod_{\{i, j\} \in g}\left(\zeta\left(Z_{i}, Z_{j}\right)-1\right) & \text { if } n \geq 2
\end{array}\right.
$$

where $\mathcal{C}_{n}$ is the set of connected subgraphs of $G_{n}$. By a standard argument, outlined in the motivation below,

$$
\ln \int e^{F(\psi, \varphi)} d \mu=\sum_{n=1}^{\infty} \frac{1}{n !} \sum_{Z_{1}, \cdots, Z_{n} \subset X} \rho^{T}\left(Z_{1}, \cdots, Z_{n}\right) \prod_{j=1}^{n} \Phi\left(Z_{j}\right)
$$

(By "ln" we just mean that the exponential of the right hand side is $\int e^{F}(\psi, \varphi) d \mu$.)

Motivation Define the value of the graph $g \subset G_{n}$ to be

$$
\operatorname{Val}(g)= \begin{cases}\sum_{Z \subset X} \Phi(Z) & \text { if } n=1 \\ \sum_{Z_{1}, \cdots, Z_{n}} \prod_{\{i, j\} \in g} C\left(Z_{i}, Z_{j}\right) \prod_{j=1}^{n} \Phi\left(Z_{j}\right) & \text { if } n>1\end{cases}
$$

where $C\left(Z_{i}, Z_{j}\right)=\zeta\left(Z_{i}, Z_{j}\right)-1$. If the connected components of $g \in \mathcal{G}_{n}$ are $g_{1}, \cdots$, $g_{m}$, then

$$
\operatorname{Val}(g)=\prod_{j=1}^{m} \operatorname{Val}\left(g_{m}\right)
$$


Directly from the definitions,

$$
\int e^{F(\psi, \varphi)} d \mu=1+\sum_{n=1}^{\infty} \frac{1}{n !} \sum_{g \subset G_{n}} \operatorname{Val}(g)
$$

On the other hand, the exponential of the right hand side of (1.54) is

$$
\exp \left\{\sum_{n=1}^{\infty} \frac{1}{n !} \sum_{g \in \mathcal{C}_{n}} \operatorname{Val}(g)\right\}=\prod_{n=1}^{\infty} \prod_{g \in \mathcal{C}_{n}} e^{\frac{1}{n !} \operatorname{Val}(g)}
$$

If you expand out the exponential and the two products, you will get the sum of the values of all graphs, with the value of each graph given as the product of the values of its connected components. To complete the proof that the right hand sides of (1.55) and (1.56) are equal, you just have to check carefully that the combinatorial coefficients match up. See, for example, (Salmhofer, 1999, §2.4).

Equation (1.54) provides a formula for $f(\psi)=\ln \int e^{F(\psi, \varphi)} d \mu(\varphi)$. We now just unravel all of the definitions to extract the coefficient system $\{a(\overrightarrow{\mathbf{x}})\}_{\overrightarrow{\mathbf{x}} \in \mathcal{X}}$, of (1.47), for $f(\psi)$. Recall from (1.53) that

$$
\Phi(Z)=\sum_{k=1}^{\infty} \frac{1}{k !} \sum_{\left(\overrightarrow{\mathbf{y}}_{1}, \cdots, \overrightarrow{\mathbf{y}}_{k}\right) \in \mathcal{C}(Z)} \alpha\left(\overrightarrow{\mathbf{y}}_{1}\right) \cdots \alpha\left(\overrightarrow{\mathbf{y}}_{k}\right) \int \varphi\left(\overrightarrow{\mathbf{y}}_{1}\right) \cdots \varphi\left(\overrightarrow{\mathbf{y}}_{k}\right) d \mu(\varphi)
$$

and substitute in

$$
\alpha(\overrightarrow{\mathbf{y}})=\sum_{\overrightarrow{\mathbf{x}} \in \mathcal{X}} A(\overrightarrow{\mathbf{x}}, \overrightarrow{\mathbf{y}}) \psi(\overrightarrow{\mathbf{x}})
$$

to give

$$
\Phi(Z)=\sum_{k=1}^{\infty} \frac{1}{k !} \sum_{\substack{\left(\vec{y}_{1}, \cdots, \vec{y}_{k}\right) \in \mathcal{C}(Z) \\ \overrightarrow{\mathbf{x}}_{1}, \cdots, \mathbf{x}_{k} \in \mathcal{X}}} A\left(\overrightarrow{\mathbf{x}}_{1}, \overrightarrow{\mathbf{y}}_{1}\right) \cdots A\left(\overrightarrow{\mathbf{x}}_{k}, \overrightarrow{\mathbf{y}}_{k}\right) \psi\left(\overrightarrow{\mathbf{x}}_{1}\right) \cdots \psi\left(\overrightarrow{\mathbf{x}}_{k}\right) \int \varphi\left(\overrightarrow{\mathbf{y}}_{1}\right) \cdots \varphi\left(\overrightarrow{\mathbf{y}}_{k}\right) d \mu(\varphi)
$$

So, if we set, for each $(\overrightarrow{\mathbf{x}}, \overrightarrow{\mathbf{y}}) \in \mathcal{X}^{2}$,

$$
\tilde{A}(\overrightarrow{\mathbf{x}}, \overrightarrow{\mathbf{y}})=\sum_{k=1}^{\infty} \frac{1}{k !} \sum_{\substack{\left(\overrightarrow{\mathbf{y}}_{1}, \ldots, \overrightarrow{\mathbf{y}}_{k}\right) \in \mathcal{C}\left(\text { supp } \\ \overrightarrow{\mathbf{y}}_{1} \ldots \ldots, \overrightarrow{\mathbf{y}}\right)}} \sum_{\substack{\overrightarrow{\mathbf{x}}_{1}, \ldots, \overrightarrow{\mathbf{x}}_{k}=\overrightarrow{\mathbf{y}} \\ \overrightarrow{\mathbf{x}}_{1} \circ \ldots \circ \overrightarrow{\mathbf{x}}_{k}=\overrightarrow{\mathbf{x}}}} A\left(\overrightarrow{\mathbf{x}}_{1}, \overrightarrow{\mathbf{y}}_{1}\right) \cdots A\left(\overrightarrow{\mathbf{x}}_{k}, \overrightarrow{\mathbf{y}}_{k}\right) \int \varphi(\overrightarrow{\mathbf{y}}) d \mu(\varphi)
$$

we have

$$
\Phi(Z)(\psi)=\sum_{\substack{(\overrightarrow{\mathbf{x}}, \overrightarrow{\mathbf{y}}) \in \mathcal{X}^{2} \\ \operatorname{supp} \overrightarrow{\mathbf{y}}=Z}} \tilde{A}(\overrightarrow{\mathbf{x}}, \overrightarrow{\mathbf{y}}) \psi(\overrightarrow{\mathbf{x}})
$$

Recall, from (1.54), that

$$
\ln \int e^{F(\psi, \varphi)} d \mu=\sum_{n=1}^{\infty} \frac{1}{n !} \sum_{Z_{1}, \cdots, Z_{n} \subset X} \rho^{T}\left(Z_{1}, \cdots, Z_{n}\right) \prod_{j=1}^{n} \Phi\left(Z_{j}\right)
$$


Therefore,

$$
\ln \int e^{F(\psi, \varphi)} d \mu(\varphi)=\sum_{\overrightarrow{\mathbf{x}} \in \mathcal{X}} a(\overrightarrow{\mathbf{x}}) \psi(\overrightarrow{\mathbf{x}})
$$

where, for $\overrightarrow{\mathrm{x}} \in \mathcal{X}$,

$$
a(\overrightarrow{\mathbf{x}})=\sum_{n=1}^{\infty} \frac{1}{n !} \sum_{\substack{\overrightarrow{\mathbf{x}}_{1}, \ldots, \vec{x}_{n} \in \mathcal{X} \\ \overrightarrow{\mathbf{x}}_{1} 0 \ldots, 0 \overrightarrow{\mathbf{x}}_{n}=\overrightarrow{\mathbf{x}}}} \sum_{\overrightarrow{\mathbf{y}}_{1}, \cdots, \overrightarrow{\mathbf{y}}_{n} \in \mathcal{X}} \rho^{T}\left(\operatorname{supp} \overrightarrow{\mathbf{y}}_{1}, \cdots, \operatorname{supp} \overrightarrow{\mathbf{y}}_{n}\right) \prod_{j=1}^{n} \tilde{A}\left(\overrightarrow{\mathbf{x}}_{j}, \overrightarrow{\mathbf{y}}_{j}\right)
$$

Also

$$
f(\psi)=\ln \frac{\int e^{F(\psi, \varphi)} d \mu(\varphi)}{\int e^{F(0, \varphi)} d \mu(\varphi)}=\sum_{\substack{\vec{x} \in \mathcal{X} \\ n(\overrightarrow{\mathbf{x}})>0}} a(\overrightarrow{\mathbf{x}}) \psi(\overrightarrow{\mathbf{x}})
$$

Now the $a(\overrightarrow{\mathbf{x}})$ of (1.58) might not be invariant under permutations of the components of $\overrightarrow{\mathrm{x}}$. We can of course symmetrize, but that will not be necessary for doing the estimates.

This brings us to the end of the algebraic part of the proof. We next specify the class of norms that are used in Theorem 1.29. This class generalizes the norms of (1.26) and (1.27).

\subsubsection{Norms}

Definition 1.23 (Weight System for One Field) A weight system for one field is a function $w: \mathcal{X} \rightarrow(0, \infty)$ that satisfies:

(a) $w(\overrightarrow{\mathbf{x}})$ is invariant under permutations of the components of $\overrightarrow{\mathbf{x}}$.

(b)

for all $\overrightarrow{\mathbf{x}}, \overrightarrow{\mathbf{x}}^{\prime} \in \mathcal{X}$ with $\operatorname{supp}(\overrightarrow{\mathbf{x}}) \cap \operatorname{supp}\left(\overrightarrow{\mathbf{x}}^{\prime}\right) \neq \emptyset$.

\section{Example 1.24 (Weight Systems)}

(a) If $\kappa: X \rightarrow(0, \infty)$ (called a weight factor) then

$$
w(\overrightarrow{\mathbf{x}})=\kappa(\overrightarrow{\mathbf{x}})=\prod_{\ell=1}^{n(\overrightarrow{\mathbf{x}})} \kappa\left(\mathbf{x}_{\ell}\right)
$$

is a weight system for one field.

(b) Let $d: X \times X \rightarrow \mathbb{R}_{\geq 0}$ be a metric. The length of a tree $T$ with vertices in $X$ is the sum of the lengths of all edges of $T$ (where the length of an edge is the distance between its vertices). For a subset $S \subset X$, denote by $\tau(S)$ the length of the shortest tree in $X$ whose set of vertices contains $S$. Then

$$
w(\overrightarrow{\mathbf{x}})=e^{\tau(\operatorname{supp}(\overrightarrow{\mathbf{x}}))}
$$

is a weight system for one field.

(c) If $w_{1}(\overrightarrow{\mathbf{x}})$ and $w_{2}(\overrightarrow{\mathbf{x}})$ are weight systems for one field, then so is

$$
w_{3}(\overrightarrow{\mathbf{x}})=w_{1}(\overrightarrow{\mathbf{x}}) w_{2}(\overrightarrow{\mathbf{x}})
$$


Definition 1.25 (Norms for functions of one field) Let $f(\psi)$ be a function which is defined and analytic on a neighbourhood of the origin in $\mathbb{C}^{|X|}$. Then $f$ has a unique expansion of the form $f(\psi)=\sum_{\overrightarrow{\mathbf{x}} \in \mathcal{X}} a(\overrightarrow{\mathbf{x}}) \psi(\overrightarrow{\mathbf{x}})$ with $a(\overrightarrow{\mathbf{x}})$ invariant under permutations of the components of $\overrightarrow{\mathbf{x}}$. (We call $a=\{a(\overrightarrow{\mathbf{x}}) \mid \overrightarrow{\mathbf{x}} \in \mathcal{X}\}$ the symmetric coefficient system for $f$.) If $w(\overrightarrow{\mathbf{x}})$ is a weight system for one field, we define

$$
\|f\|_{w}=\|a\|_{w} \equiv \sum_{n \geq 0} \max _{\substack{1 \leq i \leq n \\ \mathbf{z} \in \bar{X}}} \sum_{\substack{\overrightarrow{\mathbf{x}} \in X^{n} \\ \mathbf{x}_{i}=\mathbf{z}}} w(\overrightarrow{\mathbf{x}})|a(\overrightarrow{\mathbf{x}})|
$$

Here $\mathbf{x}_{i}$ is the $i^{\text {th }}$ component of the $n$-tuple $\overrightarrow{\mathbf{x}}$. The term in the above sum with $n=0$ is simply $w(-)|a(-)|$ where - denotes the 0-tuple.

\section{Remark 1.26 If}

$$
f(\psi)=\sum_{\overrightarrow{\mathbf{x}} \in \mathcal{X}} a(\overrightarrow{\mathbf{x}}) \psi(\overrightarrow{\mathbf{x}})
$$

with a $(\overrightarrow{\mathbf{x}})$ not necessarily invariant under permutations of the components of $\overrightarrow{\mathbf{x}}$, then

$$
\|f\|_{w} \leq\|a\|_{w} \equiv \sum_{n \geq 0} \max _{\substack{1 \leq i \leq n \\ \mathbf{z} \in X}} \sum_{\substack{\overrightarrow{\mathbf{x}} \in X^{n} \\ \mathbf{x}_{i}=\mathbf{z}}} w(\overrightarrow{\mathbf{x}})|a(\overrightarrow{\mathbf{x}})|
$$

Definition 1.27 (Weight System for Two Fields) A weight system for two fields is a function $W: \mathcal{X}^{2} \rightarrow(0, \infty)$ that satisfies:

(a) $W(\overrightarrow{\mathbf{x}}, \overrightarrow{\mathbf{y}})$ is invariant under permutations of the components of $\overrightarrow{\mathbf{x}}$ and is invariant under permutations of the components of $\overrightarrow{\mathbf{y}}$.

(b) $\quad W\left(\overrightarrow{\mathbf{x}} \circ \overrightarrow{\mathbf{x}}^{\prime}, \overrightarrow{\mathbf{y}} \circ \overrightarrow{\mathbf{y}}^{\prime}\right) \leq W(\overrightarrow{\mathbf{x}}, \overrightarrow{\mathbf{y}}) W\left(\overrightarrow{\mathbf{x}}^{\prime}, \overrightarrow{\mathbf{y}}^{\prime}\right)$

whenever $\operatorname{supp}(\overrightarrow{\mathbf{x}}, \overrightarrow{\mathbf{y}}) \cap \operatorname{supp}\left(\overrightarrow{\mathbf{x}}^{\prime}, \overrightarrow{\mathbf{y}}^{\prime}\right) \neq \emptyset$.

Definition 1.28 (Norms for functions of two fields)

Let

$$
F(\psi, \varphi)=\sum_{(\overrightarrow{\mathbf{x}}, \overrightarrow{\mathbf{y}}) \in \mathcal{X}^{2}} A(\overrightarrow{\mathbf{x}}, \overrightarrow{\mathbf{y}}) \psi(\overrightarrow{\mathbf{x}}) \varphi(\overrightarrow{\mathbf{y}})
$$

with $A(\overrightarrow{\mathbf{x}}, \overrightarrow{\mathbf{y}})$ invariant under permutations of the components of $\overrightarrow{\mathbf{x}}$ and under permutations of the components of $\overrightarrow{\mathbf{y}}$. If $W(\overrightarrow{\mathbf{x}}, \overrightarrow{\mathbf{y}})$ is a weight system for two fields, we define

$$
\|F\|_{W}=\|A\|_{W} \equiv \sum_{n, m \geq 0} \max _{\substack{1 \leq i \leq n+m \\ \mathbf{z} \in X}} \sum_{\substack{(\overrightarrow{\mathbf{x}}, \overrightarrow{\mathbf{y}}) \in X^{n} \times X^{m} \\(\vec{x}, \overrightarrow{\mathbf{y}})_{i}=\mathbf{z}}} W(\overrightarrow{\mathbf{x}}, \overrightarrow{\mathbf{y}})|A(\overrightarrow{\mathbf{x}}, \overrightarrow{\mathbf{y}})|
$$

Here $(\overrightarrow{\mathbf{x}}, \overrightarrow{\mathbf{y}})_{i}$ is the $i^{\text {th }}$ component of the $(n+m)$-tuple $(\overrightarrow{\mathbf{x}}, \overrightarrow{\mathbf{y}})$. The term in the above sum with $n=m=0$ is simply $W(-,-)|A(-,-)|$. 
50 The Temporal Ultraviolet Limit

\subsubsection{Review of the Main Theorem}

Recall that

- $X(=$ space $)$ is a finite set and

- $d \mu_{0}(z)$ is a normalized measure on $\mathbb{C}$ that is supported in $|z| \leq r$ for some constant $r$ and

- we endow $\mathbb{C}^{X}$ with the ultralocal product measure

$$
d \mu(\varphi)=\prod_{\mathbf{x} \in X} d \mu_{0}(\varphi(\mathbf{x}))
$$

We now have all of the definitions required to state

Theorem 1.29 Let $w$ and $W$ be weight systems for 1 and 2 fields, respectively, that obey

$$
W(\overrightarrow{\mathbf{x}}, \overrightarrow{\mathbf{y}}) \geq(4 r)^{n(\overrightarrow{\mathbf{y}})} w(\overrightarrow{\mathbf{x}})
$$

Let $F: \mathbb{C}^{|X|} \times \mathbb{C}^{|X|} \rightarrow \mathbb{C}$ be analytic on a neighbourhood of the origin. If $F(\psi, \varphi)$ obeys $\|F\|_{W}<\frac{1}{16}$, then there is an analytic function $f(\psi)$ such that

$$
\frac{\int e^{F(\psi, \varphi)} d \mu(\varphi)}{\int e^{F(0, \varphi)} d \mu(\varphi)}=e^{f(\psi)}
$$

and

$$
\|f\|_{w} \leq \frac{\|F\|_{W}}{1-16\|F\|_{W}}
$$

\subsubsection{Outline of the Proof of Theorem 1.29 - Bounds}

Step 1 - organizing the sums. Recall, from (1.58), that

$$
a(\overrightarrow{\mathbf{x}})=\sum_{n=1}^{\infty} \frac{1}{n !} \sum_{\substack{\overrightarrow{\mathbf{x}}_{1}, \cdots, \overrightarrow{\mathbf{x}}_{n} \in \mathcal{X} \\ \overrightarrow{\mathbf{x}}_{1} 0 \cdots, \overrightarrow{\mathbf{x}}_{n}=\overrightarrow{\mathbf{x}}}} \sum_{\overrightarrow{\mathbf{y}}_{1}, \cdots, \overrightarrow{\mathbf{y}}_{n} \in \mathcal{X}} \rho^{T}\left(\operatorname{supp} \overrightarrow{\mathbf{y}}_{1}, \cdots, \operatorname{supp} \overrightarrow{\mathbf{y}}_{n}\right) \prod_{j=1}^{n} \tilde{A}\left(\overrightarrow{\mathbf{x}}_{j}, \overrightarrow{\mathbf{y}}_{j}\right)
$$

The bound

$$
\mid \rho^{T}\left(\operatorname{supp} \overrightarrow{\mathbf{y}}_{1}, \cdots, \text { supp } \overrightarrow{\mathbf{y}}_{n}\right) \mid \leq \#\left\{\text { spanning trees in } G\left(\overrightarrow{\mathbf{y}}_{1}, \cdots, \overrightarrow{\mathbf{y}}_{n}\right)\right\}
$$

is due to Rota (Rota, 1964). For a simple proof see (Simon, 1993, Theorem V.7.A.6). A spanning tree for a graph is just a tree with the same set of vertices as the graph. Hence

$$
\begin{aligned}
|a(\overrightarrow{\mathbf{x}})| & \leq \sum_{n=1}^{\infty} \frac{1}{n !} \sum_{\substack{\overrightarrow{\mathbf{x}}_{1}, \cdots, \overrightarrow{\mathbf{x}}_{n} \in \mathcal{X} \\
\overrightarrow{\mathbf{x}}_{1} 0 \cdots, \overrightarrow{\mathbf{x}}_{n}=\overrightarrow{\mathbf{y}}_{1}, \cdots, \overrightarrow{\mathbf{y}}_{n} \in \mathcal{X}}} \sum_{\substack{T \text { spanning tree } \\
\text { for } G\left(\overrightarrow{\mathbf{y}}_{1}, \cdots, \overrightarrow{\mathbf{y}}_{n}\right)}} \prod_{j=1}^{n}\left|\tilde{A}\left(\overrightarrow{\mathbf{x}}_{j}, \overrightarrow{\mathbf{y}}_{j}\right)\right| \\
& \leq \sum_{n=1}^{\infty} \frac{1}{n !} \sum_{\substack{T \\
\text { labelled tree with } \\
\text { vertices } 1, \cdots, n}}|\tilde{\mathbf{y}} \in \mathcal{X}|_{T}(\overrightarrow{\mathbf{x}}, \overrightarrow{\mathbf{y}})
\end{aligned}
$$


where

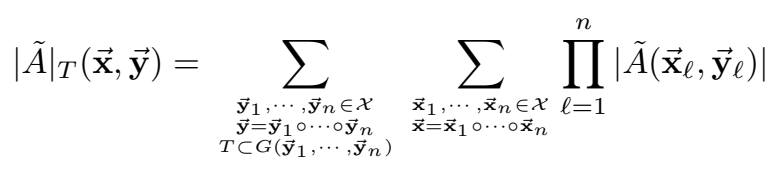

Recall, from (1.57), that

$$
\tilde{A}(\overrightarrow{\mathbf{x}}, \overrightarrow{\mathbf{y}})=\sum_{k=1}^{\infty} \frac{1}{k !} \sum_{\substack{\left(\overrightarrow{\mathbf{y}}_{1}, \ldots, \overrightarrow{\mathbf{y}}_{k}\right) \in \mathcal{C}\left(\text { supp } \\ \overrightarrow{\mathbf{y}}_{1} \ldots \ldots, \overrightarrow{\mathbf{y}}\right)}} \sum_{\substack{\overrightarrow{\mathbf{x}}_{1}, \ldots, \overrightarrow{\mathbf{x}}_{k}=\overrightarrow{\mathbf{y}} \\ \overrightarrow{\mathbf{x}}_{1} \circ \ldots \circ \overrightarrow{\mathbf{x}}_{k}=\overrightarrow{\mathbf{x}}}} A\left(\overrightarrow{\mathbf{x}}_{1}, \overrightarrow{\mathbf{y}}_{1}\right) \cdots A\left(\overrightarrow{\mathbf{x}}_{k}, \overrightarrow{\mathbf{y}}_{k}\right) \int \varphi(\overrightarrow{\mathbf{y}}) d \mu(\varphi)
$$

For each $\left(\overrightarrow{\mathbf{y}}_{1}, \cdots, \overrightarrow{\mathbf{y}}_{k}\right), G\left(\overrightarrow{\mathbf{y}}_{1}, \cdots, \overrightarrow{\mathbf{y}}_{k}\right)$ is connected and hence contains at least one tree. So

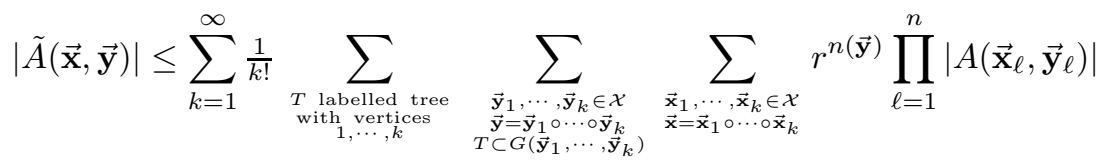

$$
\begin{aligned}
& =\sum_{k=1}^{\infty} \frac{1}{k !} \sum_{\substack{T \text { labelled tree } \\
\text { with vertices } \\
1, \cdots, k}} r^{n(\overrightarrow{\mathbf{y}})}|A|_{T}(\overrightarrow{\mathbf{x}}, \overrightarrow{\mathbf{y}})
\end{aligned}
$$

where

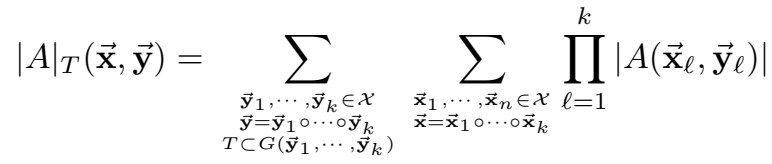

Step 2 - bound on $B_{T}$.

Lemma 1.30 Let $\omega$ be an arbitrary weight system for two fields and define the weight system $\omega^{\prime}$ by

$$
\omega^{\prime}(\overrightarrow{\mathbf{x}}, \overrightarrow{\mathbf{y}})=2^{n(\overrightarrow{\mathbf{y}})} \omega(\overrightarrow{\mathbf{x}}, \overrightarrow{\mathbf{y}})
$$

Let $T$ be a labelled tree with vertices $1, \cdots, n$ and coordination numbers $d_{1}, \cdots, d_{n}$ (meaning that vertex $j$ has $d_{j}$ lines attached to it). Let $B$ be any (not necessarily symmetric) coefficient system for two fields with $B(-,-)=0$. We define a new coefficient system $B_{T}$ by

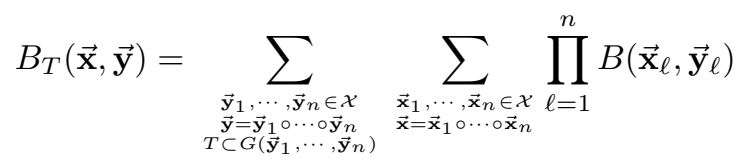

Then

$$
\left\|B_{T}\right\|_{\omega} \leq d_{1} ! \cdots d_{n} !\|B\|_{\omega^{\prime}}^{n}
$$


Outline of proof (The details are in (Balaban et al., 2009, §III).)

\section{Ingredient 1:}

- For each $1 \leq \ell \leq n$, think of $\left(\overrightarrow{\mathbf{x}}_{\ell}, \overrightarrow{\mathbf{y}}_{\ell}\right)$ as the locations of (two species of) stars in a galaxy.

- In computing $\left\|B_{T}\right\|_{\omega}=\sum_{N, M \geq 0} \max _{\substack{1 \leq i \leq N+M \\ \mathbf{z} \in X}} \sum_{\substack{\left(\overrightarrow{\mathbf{x}}, \vec{y} \in X^{N} \times X^{M} \\(\overrightarrow{\mathbf{x}}, \overrightarrow{\mathbf{y}})_{i}=\mathbf{z}\right.}} \omega(\overrightarrow{\mathbf{x}}, \overrightarrow{\mathbf{y}})\left|B_{T}(\overrightarrow{\mathbf{x}}, \overrightarrow{\mathbf{y}})\right|$, we must hold fixed the location of one star (the $i^{\text {th }}$ ) and sum over the locations of all other stars. Suppose, for example, that we have chosen $i=1$ so that the fixed star is in galaxy $\ell=1$.

- View 1 as the root of the tree $T$.

- Then the set of vertices of $T$ is endowed with a natural partial ordering under which 1 is the smallest vertex.

- For each vertex $2 \leq \ell \leq n$, denote by $\pi(\ell)$ the predecessor vertex of $\ell$ under this partial ordering, as illustrated in Figure 1.6.

$$
\begin{aligned}
& \pi(7)=\pi(3)=\pi(4)=2 \\
& \begin{array}{l}
\pi(2)=\pi(5)=6 \\
\pi(6)=1
\end{array}
\end{aligned}
$$

Fig. 1.6 A Sample Tree Partial Ordering

- The condition that $T \subset G\left(\overrightarrow{\mathbf{y}}_{1}, \cdots, \overrightarrow{\mathbf{y}}_{n}\right)$ ensures that, for each $2 \leq \ell \leq n$, the support of $\overrightarrow{\mathbf{y}}_{\ell}$ intersects the support of $\overrightarrow{\mathbf{y}}_{\pi(\ell)}$, so that at least one of the $n\left(\overrightarrow{\mathbf{y}}_{\ell}\right)$ components of $\overrightarrow{\mathbf{y}}_{\ell}$ takes the same value (in $X$ ) as some component of $\overrightarrow{\mathbf{y}}_{\pi(\ell)}$.

- Write $n\left(\overrightarrow{\mathbf{y}}_{\ell}\right)=n_{\ell}$.

- The product over $2 \leq \ell \leq n$ of the number of choices of which $\overrightarrow{\mathbf{y}}$-star in galaxy $\ell$ is at the same location of which $\overrightarrow{\mathbf{y}}$-star in galaxy $\pi(\ell)$ is

$$
\left.\prod_{\ell=2}^{n}\left[n_{\ell} n_{\pi(\ell)}\right)\right]=\prod_{\ell=1}^{n} n_{\ell}^{d_{\ell}}=\prod_{\ell=1}^{n} \frac{n_{\ell}^{d_{\ell}}}{d_{\ell} !} d_{\ell} ! \leq d_{1} ! \cdots d_{n} ! \prod_{\ell=1}^{n} 2^{n_{\ell}}
$$

by using first year calculus and Stirling. (Alternatively, just use $\frac{n^{d}}{d !} \leq e^{n}$. This gives a slightly weaker theorem, but the change is insignificant.)

Ingredient 2:

- Since $T$ is connected,

$$
\omega(\overrightarrow{\mathbf{x}}, \overrightarrow{\mathbf{y}}) \leq \prod_{\ell=1}^{n} \omega\left(\overrightarrow{\mathbf{x}}_{\ell}, \overrightarrow{\mathbf{y}}_{\ell}\right)
$$

for all $\overrightarrow{\mathbf{x}}_{1}, \cdots, \overrightarrow{\mathbf{x}}_{n} \in \mathcal{X}$ and $\overrightarrow{\mathbf{y}}_{1}, \cdots, \overrightarrow{\mathbf{y}}_{n} \in \mathcal{X}$ under consideration, by the second condition of Definition 1.27. So we may absorb each factor $\omega\left(\overrightarrow{\mathbf{x}}_{\ell}, \overrightarrow{\mathbf{y}}_{\ell}\right)$ into $B\left(\overrightarrow{\mathbf{x}}_{\ell}, \overrightarrow{\mathbf{y}}_{\ell}\right)$ and it suffices to consider $\omega=1$. 


\section{Ingredient 3:}

- Iteratively apply

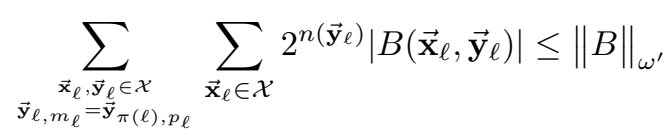

starting with the largest $\ell$ 's, in the partial ordering of $T$, and ending with $\ell=1$.

(For $\ell=1$, substitute $\overrightarrow{\mathbf{x}}_{1,1}=\mathbf{x}$ for $\overrightarrow{\mathbf{y}}_{\ell, m_{\ell}}=\overrightarrow{\mathbf{y}}_{\pi(\ell), p_{\ell}}$. )

Step 3 - sum over $n$ (or $k$ ) and $T$.

Lemma 1.31 Let $0<\varepsilon<\frac{1}{8}$. Then

$$
\sum_{n=1}^{\infty} \frac{1}{n !} \sum_{\substack{d_{1}, \cdots, d_{n} \\ d_{1}+\cdots+d_{n}=2(n-1)}} \sum_{\substack{\text { Tith labelled tree } \\ \text { numberd dination } \\ \text { numbers } \mathrm{d}_{1}, \cdots, d_{n}}} d_{1} ! \cdots d_{n} ! \varepsilon^{n} \leq \frac{\varepsilon}{1-8 \varepsilon}
$$

Proof Each line of a tree is connected to exactly two vertices. So the sum, $d_{1}+\cdots+d_{n}$, of all coordination numbers is exactly twice the number of lines in the tree. The number of lines in a tree of $n$ vertices is exactly $n=-1$. So we must have $d_{1}+\cdots+d_{n}=2(n-1)$. That accounts for the condition on the second sum.

By the Cayley formula, the number of labelled trees on $n \geq 2$ vertices with specified coordination numbers $\left(d_{1}, d_{2}, \cdots, d_{n}\right)$ is

$$
\frac{(n-2) !}{\prod_{j=1}^{n}\left(d_{j}-1\right) !}
$$

Therefore

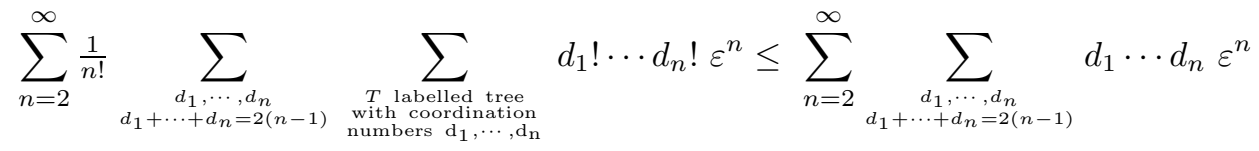

The number of possible choices of coordination numbers $\left(d_{1}, d_{2}, \cdots, d_{n}\right)$ subject to the constraint $d_{1}+d_{2}+\cdots+d_{n}=2(n-1)$ is

$$
\left(\begin{array}{c}
2(n-1)-1 \\
n-1
\end{array}\right)=\left(\begin{array}{c}
2 n-3 \\
n-1
\end{array}\right) \leq 2^{2 n-3}
$$

and $d_{1} \cdots d_{n} \leq 2^{n}$. (Any maximizer must have $d_{j} \leq 2$ for every $1 \leq j \leq n$.) Therefore

$$
\sum_{n=2}^{\infty} \frac{1}{n !} \sum_{\substack{d_{1}, \cdots, d_{n} \\ d_{1}+\cdots+d_{n}=2(n-1)}} \sum_{\substack{\text { Tith labelled tree } \\ \text { numberdination } \\ \text { numbers } d_{1}, \cdots, d_{n}}} d_{1} ! \cdots d_{n} ! \varepsilon^{n} \leq \sum_{n=2}^{\infty} 2^{2 n-3} 2^{n} \varepsilon^{n}=\frac{8 \varepsilon^{2}}{1-8 \varepsilon}
$$

For $n=1, d_{1}=0$ and the number of trees is 1 , so the $n=1$ term is $\varepsilon$. So the full sum is bounded by $\varepsilon+\frac{8 \varepsilon^{2}}{1-8 \varepsilon}=\frac{\varepsilon}{1-8 \varepsilon}$. 


\section{The Temporal Ultraviolet Limit}

Step 4 - bound on $\|a\|$ in terms of $\|\tilde{A}\|$. We introduce, for each $\sigma>0$, the auxiliary weight system

$$
W_{\sigma}(\overrightarrow{\mathbf{x}}, \overrightarrow{\mathbf{y}})=W(\overrightarrow{\mathbf{x}}, \overrightarrow{\mathbf{y}})\left(\frac{\sigma}{4 r}\right)^{n(\overrightarrow{\mathbf{y}})}
$$

Clearly

$$
W_{4 r}(\overrightarrow{\mathbf{x}}, \overrightarrow{\mathbf{y}})=W(\overrightarrow{\mathbf{x}}, \overrightarrow{\mathbf{y}}) \quad \text { and } \quad w(\overrightarrow{\mathbf{x}}) \leq W_{1}(\overrightarrow{\mathbf{x}}, \overrightarrow{\mathbf{y}})
$$

for all $(\overrightarrow{\mathbf{x}}, \overrightarrow{\mathbf{y}}) \in \mathcal{X}^{2}$.

We now prove

$$
\|a\|_{w} \leq \frac{\|\tilde{A}\|_{W_{2}}}{1-8\|\tilde{A}\|_{W_{2}}}
$$

Recall from (1.60) that

$$
|a(\overrightarrow{\mathbf{x}})| \leq \sum_{n=1}^{\infty} \frac{1}{n !} \sum_{\substack{\text { labelled tree with } \\ \text { vertices } 1, \ldots, n}} \sum_{\overrightarrow{\mathbf{y}} \in \mathcal{X}}|\tilde{A}|_{T}(\overrightarrow{\mathbf{x}}, \overrightarrow{\mathbf{y}})
$$

Therefore, by (1.61) and Lemma 1.30, with $\omega=W_{1}$ and $\omega^{\prime}=W_{2}$,

$$
\begin{aligned}
& \|a\|_{w} \leq \sum_{n=1}^{\infty} \frac{1}{n !} \sum_{\substack{T \text { labelled tree with } \\
\text { vertices } 1, \cdots, n}}\left\||\tilde{A}|_{T}\right\|_{W_{1}} \\
& \leq \sum_{n=1}^{\infty} \frac{1}{n !} \sum_{\substack{d_{1}, \cdots, d_{n} \\
d_{1}+\cdots+d_{n}=2(n-1)}} \sum_{\begin{array}{c}
T \text { labelled tree } \\
\text { with cordination } \\
\text { numbers } \mathrm{d}_{1}, \cdots, \mathrm{d}_{\mathrm{n}}
\end{array}}\left\||\tilde{A}|_{T}\right\|_{W_{1}}
\end{aligned}
$$

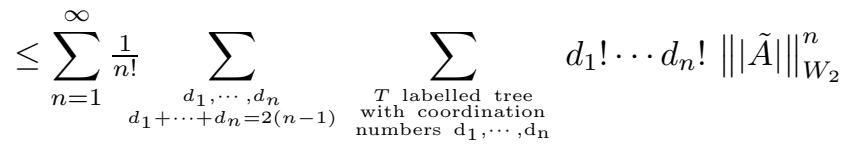

Now apply Lemma 1.31 with $\varepsilon=\|\mid \tilde{A}\|_{W_{2}}=\|\tilde{A}\|_{W_{2}}$ to get

$$
\|a\|_{w} \leq \frac{\|\tilde{A}\|_{W_{2}}}{1-8\|\tilde{A}\|_{W_{2}}}
$$

Step 5 - bound on $\|\tilde{A}\|$ in terms of $\|A\|$. We now prove

$$
\|\tilde{A}\|_{W_{2}} \leq \frac{\|A\|_{W}}{1-8\|A\|_{W}}=\frac{\|F\|_{W}}{1-8\|F\|_{W}}
$$

Note that combining (1.62) and (1.63) yields the final bound

$$
\|f\|_{w} \leq\|a\|_{w} \leq \frac{\|\tilde{A}\|_{W_{2}}}{1-8\|\tilde{A}\|_{W_{2}}} \leq \frac{\frac{\|F\|_{W}}{1-8\|F\|_{W}}}{1-8 \frac{\|F\|_{W}}{1-8\|F\|_{W}}}=\frac{\|F\|_{W}}{1-16\|F\|_{W}}
$$

Recall from (1.60') that 


$$
|\tilde{A}(\overrightarrow{\mathbf{x}}, \overrightarrow{\mathbf{y}})| \leq \sum_{k=1}^{\infty} \frac{1}{k !} \sum_{\substack{T \\
\begin{array}{l}
\text { labelled tree with } \\
\text { vertices } 1, \cdots, k
\end{array}}} r^{n(\overrightarrow{\mathbf{y}})}|A|_{T}(\overrightarrow{\mathbf{x}}, \overrightarrow{\mathbf{y}})
$$

By construction, $\left\|r^{n(\overrightarrow{\mathbf{y}})}|A|_{T}(\overrightarrow{\mathbf{x}}, \overrightarrow{\mathbf{y}})\right\|_{W_{2}}=\left\||A|_{T}\right\|_{W_{2 r}}$. Hence, by Lemma 1.30, with $\omega=W_{2 r}$ followed by Lemma 1.31 ,

$$
\begin{aligned}
& \|\tilde{A}\|_{W_{2}} \leq \sum_{k=1}^{\infty} \frac{1}{k !} \sum_{\substack{T \\
\begin{array}{c}
\text { labelled tree with } \\
\text { vertices } 1, \cdots, k
\end{array}}}\left\||A|_{T}\right\|_{W_{2 r}}
\end{aligned}
$$

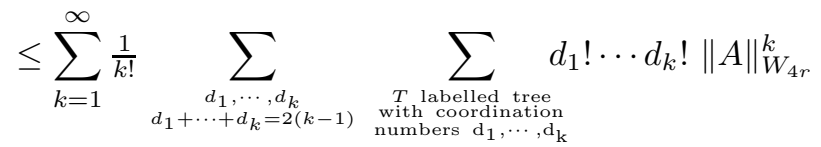

$$
\begin{aligned}
& \leq \frac{\|A\|_{W}}{1-8\|A\|_{W}}
\end{aligned}
$$

since $W_{4 r}=W$. This gives (1.63).

\subsubsection{Changes of Variables}

In this subsection we provide a couple of tools that may used to prove bounds on "complicated" functions that are constructed from "simple" functions using changes of variables.

For $\kappa>0$, we denote by $w_{\kappa}$ the weight system, for functions of one field, $\psi$, with mass $m$ that associates the constant weight factor $\kappa$ to the field $\psi$. That is

$$
w_{\kappa}\left(\mathbf{x}_{1}, \cdots, \mathbf{x}_{n}\right)=e^{\mathrm{m} \tau\left(\left\{\mathbf{x}_{1}, \cdots, \mathbf{x}_{n}\right\}\right)} \kappa^{n}
$$

Similarly, for $\kappa, \lambda>0$, we denote by $w_{\kappa, \lambda}$ the weight system, for functions of two fields, $\psi$ and $\phi$, with mass $\mathrm{m}$ that associates the constant weight factor $\kappa$ to the field $\psi$ and the constant weight factor $\lambda$ to the field $\phi$. To simplify notation, we write $\|g(\psi)\|_{\kappa}$ and $\|f(\psi, \phi)\|_{\kappa, \lambda}$ for $\|g(\psi)\|_{w_{\kappa}}$ and $\|f(\psi, \phi)\|_{w_{\kappa, \lambda}}$, respectively.

Proposition 1.32 Let $g$ be an analytic function on a neighbourhood of the origin in $\mathbb{C}^{X}$.

(i) Let $J$ be an operator on $\mathbb{C}^{X}$ with kernel $J(\mathbf{x}, \mathbf{y})$. Define $\tilde{g}$ by

$$
\tilde{g}(\psi)=g(J \psi)
$$

Let $\kappa>0$ and set $\kappa^{\prime}=\kappa\|\| J \|$. ( $\|J\|$ was defined in (1.28).) Then $\|\tilde{g}\|_{\kappa} \leq\|g\|_{\kappa^{\prime}}$.

(ii) Define $f$ by

$$
f(\psi ; \phi)=g(\psi+\phi)
$$

Then $\|f\|_{\kappa, \lambda}=\|g\|_{\kappa+\lambda}$. 


\section{The Temporal Ultraviolet Limit}

Proof (i) Let $a(\overrightarrow{\mathbf{x}})$ be a symmetric coefficient system for $g$. Define, for each $n \geq 0$,

$$
\tilde{a}\left(\mathbf{x}_{1}, \cdots, \mathbf{x}_{n}\right)=\sum_{\mathbf{y}_{1}, \cdots, \mathbf{y}_{n} \in X} a\left(\mathbf{y}_{1}, \ldots, \mathbf{y}_{n}\right) \prod_{\ell=1}^{n} J\left(\mathbf{y}_{\ell}, \mathbf{x}_{\ell}\right)
$$

Then $\tilde{a}(\overrightarrow{\mathbf{x}})$ is a symmetric coefficient system for $\tilde{g}$. Since

$$
\tau\left(\left\{\mathbf{x}_{1}, \cdots, \mathbf{x}_{n}\right\}\right) \leq \tau\left(\left\{\mathbf{y}_{1}, \cdots, \mathbf{y}_{n}\right\}\right)+\sum_{\ell=1}^{n} d\left(\mathbf{y}_{\ell}, \mathbf{x}_{\ell}\right)
$$

we have

$$
e^{\mathrm{m} \tau\left(\left\{\mathbf{x}_{1}, \cdots, \mathbf{x}_{n}\right\}\right)} \leq e^{\mathrm{m} \tau\left(\left\{\mathbf{y}_{1}, \cdots, \mathbf{y}_{n}\right\}\right)} \prod_{\ell=1}^{n} e^{\mathrm{m} d\left(\mathbf{y}_{\ell}, \mathbf{x}_{\ell}\right)}
$$

and hence

$$
\begin{aligned}
& w_{\kappa}\left(\mathbf{x}_{1}, \cdots, \mathbf{x}_{n}\right)\left|\tilde{a}\left(\mathbf{x}_{1}, \cdots, \mathbf{x}_{n}\right)\right| \\
& \quad \leq \sum_{\mathbf{y}_{1}, \cdots, \mathbf{y}_{n} \in X} w_{\kappa^{\prime}}\left(\mathbf{y}_{1}, \cdots, \mathbf{y}_{n}\right)\left|a\left(\mathbf{y}_{1}, \cdots, \mathbf{y}_{n}\right)\right| \prod_{\ell=1}^{n}\left[\frac{\kappa}{\kappa^{\prime}} e^{\mathrm{m} d\left(\mathbf{y}_{\ell}, \mathbf{x}_{\ell}\right)}\left|J\left(\mathbf{y}_{\ell}, \mathbf{x}_{\ell}\right)\right|\right]
\end{aligned}
$$

We are to bound

$$
\begin{aligned}
&\|\tilde{g}\|_{\kappa}=\sum_{n \geq 0} \max _{\mathbf{x} \in X} \max _{1 \leq j \leq n} \sum_{\substack{\mathbf{x}_{1}, \cdots, \mathbf{x}_{n} \in X^{n} \\
\mathbf{x}_{j}=\mathbf{x}}} w_{\kappa}\left(\mathbf{x}_{1}, \cdots, \mathbf{x}_{n}\right)\left|\tilde{a}\left(\mathbf{x}_{1}, \cdots, \mathbf{x}_{n}\right)\right| \\
& \leq \sum_{n \geq 0} \max _{\mathbf{x} \in X} \max _{1 \leq j \leq n} \sum_{\substack{\mathbf{x}_{1}, \cdots, \mathbf{x}_{n} \in X \\
\mathbf{x}_{j}=\mathbf{x}}} \sum_{\mathbf{y}_{1}, \cdots, \mathbf{y}_{n} \in X} \\
& w_{\kappa^{\prime}}\left(\mathbf{y}_{1}, \cdots, \mathbf{y}_{n}\right)\left|a\left(\mathbf{y}_{1}, \cdots, \mathbf{y}_{n}\right)\right| \prod_{\ell=1}^{n}\left[\frac{\kappa}{\kappa^{\prime}} e^{\mathrm{m} d\left(\mathbf{y}_{\ell}, \mathbf{x}_{\ell}\right)}\left|J\left(\mathbf{y}_{\ell}, \mathbf{x}_{\ell}\right)\right|\right]
\end{aligned}
$$

Fix any $n \geq 0, \mathbf{x} \in X$ and $1 \leq j \leq n$. By the definitions of $\kappa^{\prime}$ and $\|J\|$, for each $\ell \neq j$ and $\mathbf{y}_{\ell} \in X$,

$$
\sum_{\mathbf{x}_{\ell} \in X} \frac{\kappa}{\kappa^{\prime}} e^{\mathrm{m} d\left(\mathbf{y}_{\ell}, \mathbf{x}_{\ell}\right)} J\left(\mathbf{y}_{\ell}, \mathbf{x}_{\ell}\right)=\sum_{\mathbf{x}_{\ell} \in X} \frac{1}{\|J\|} e^{\mathrm{m} d\left(\mathbf{y}_{\ell}, \mathbf{x}_{\ell}\right)} J\left(\mathbf{y}_{\ell}, \mathbf{x}_{\ell}\right) \leq 1
$$

Therefore

$$
\begin{aligned}
& \sum_{\substack{\mathbf{x}_{1}, \cdots, \mathbf{x}_{n} \in X \\
\mathbf{x}_{j}=\mathbf{x}}} \sum_{\mathbf{y}_{1}, \cdots, \mathbf{y}_{n} \in X} w_{\kappa^{\prime}}\left(\mathbf{y}_{1}, \cdots, \mathbf{y}_{n}\right)\left|a\left(\mathbf{y}_{1}, \cdots, \mathbf{y}_{n}\right)\right| \prod_{\ell=1}^{n}\left[\frac{\kappa}{\kappa^{\prime}} e^{m d\left(\mathbf{y}_{\ell}, \mathbf{x}_{\ell}\right)}\left|J\left(\mathbf{y}_{\ell}, \mathbf{x}_{\ell}\right)\right|\right] \\
& \leq \sum_{\mathbf{y} \in X} \frac{\kappa}{\kappa^{\prime}} e^{m d(\mathbf{y}, \mathbf{x})} J(\mathbf{y}, \mathbf{x}) \sum_{\substack{\mathbf{y}_{1}, \cdots, \mathbf{y}_{n} \in X \\
\mathbf{y}_{j}=\mathbf{y}}} w_{\kappa^{\prime}}\left(\mathbf{y}_{1}, \cdots, \mathbf{y}_{n}\right)\left|a\left(\mathbf{y}_{1}, \cdots, \mathbf{y}_{n}\right)\right| \\
& \leq \sum_{\mathbf{y} \in X} \frac{\kappa}{\kappa^{\prime}} e^{\mathrm{m} d(\mathbf{y}, \mathbf{x})} J(\mathbf{y}, \mathbf{x}) \max _{\mathbf{y} \in X} \sum_{\substack{\mathbf{y}_{1}, \cdots, \mathbf{y}_{n} \in X \\
\mathbf{y}_{j}=\mathbf{y}}} w_{\kappa^{\prime}}\left(\mathbf{y}_{1}, \cdots, \mathbf{y}_{n}\right)\left|a\left(\mathbf{y}_{1}, \cdots, \mathbf{y}_{n}\right)\right| \\
& \leq \max _{\mathbf{y} \in X} \sum_{\mathbf{y}_{1}, \cdots, \mathbf{y}_{n} \in X} w_{\kappa^{\prime}}\left(\mathbf{y}_{1}, \cdots, \mathbf{y}_{n}\right)\left|a\left(\mathbf{y}_{1}, \cdots, \mathbf{y}_{n}\right)\right|
\end{aligned}
$$


since, once again, $\sum_{\mathbf{y} \in X} \frac{\kappa}{\kappa^{\prime}} e^{\mathrm{m} d(\mathbf{y}, \mathbf{x})} J(\mathbf{y}, \mathbf{x})=\sum_{y \in X} \frac{1}{\|J\|} e^{\mathrm{m} d(\mathbf{y}, \mathbf{x})} J(\mathbf{y}, \mathbf{x}) \leq 1$. Consequently, (1.64) is bounded by

$$
\sum_{n \geq 0} \max _{1 \leq j \leq n} \max _{\mathbf{y} \in X} \sum_{\substack{\mathbf{y}_{1}, \ldots, \mathbf{y}_{n} \in X \\ \mathbf{y}_{j}=\mathbf{y}}} w_{\kappa^{\prime}}\left(\mathbf{y}_{1}, \cdots, \mathbf{y}_{n}\right)\left|a\left(\mathbf{y}_{1}, \cdots, \mathbf{y}_{n}\right)\right|=\|g\|_{\kappa^{\prime}}
$$

This proves part (i) of the Proposition.

(ii) Let $a(\overrightarrow{\mathbf{u}})$ be a symmetric coefficient system for $g$. Since $a$ is invariant under permutation of its $\overrightarrow{\mathbf{u}}$ components,

$$
g(\psi+\phi)=\sum_{\overrightarrow{\mathbf{u}} \in \mathcal{X}} a(\overrightarrow{\mathbf{u}})(\psi+\phi)(\overrightarrow{\mathbf{u}})=\sum_{\overrightarrow{\mathbf{x}}, \overrightarrow{\mathbf{y}} \in \mathcal{X}} a(\overrightarrow{\mathbf{x}} \circ \overrightarrow{\mathbf{y}})\left(\begin{array}{c}
n(\overrightarrow{\mathbf{x}})+n(\overrightarrow{\mathbf{y}}) \\
n(\overrightarrow{\mathbf{y}})
\end{array}\right) \psi(\overrightarrow{\mathbf{x}}) \phi(\overrightarrow{\mathbf{y}})
$$

so that

$$
a_{+}(\overrightarrow{\mathbf{x}} ; \overrightarrow{\mathbf{y}})=a(\overrightarrow{\mathbf{x}} \circ \overrightarrow{\mathbf{y}})\left(\begin{array}{c}
n(\overrightarrow{\mathbf{x}})+n(\overrightarrow{\mathbf{y}}) \\
n(\overrightarrow{\mathbf{y}})
\end{array}\right)
$$

is a symmetric coefficient system for $f$. We have

$$
\begin{aligned}
& \|f\|_{\kappa, \lambda}=\sum_{k, \ell \geq 0} \max _{\mathbf{p} \in X} \max _{1 \leq i \leq k+\ell} \sum_{\substack{\overrightarrow{\mathbf{x}} \in X^{k}, \overrightarrow{\mathbf{y}} \in X^{\ell} \\
(\vec{x}, \overrightarrow{\mathbf{y}}) i=\mathbf{p}}} w_{\kappa, \lambda}(\overrightarrow{\mathbf{x}} ; \overrightarrow{\mathbf{y}})\left|a_{+}(\overrightarrow{\mathbf{x}} ; \overrightarrow{\mathbf{y}})\right| \\
& =\sum_{k, \ell \geq 0} \max _{\mathbf{p} \in X} \max _{1 \leq i \leq k+\ell} \sum_{\substack{\overrightarrow{\mathbf{x}} \in X^{k}, \overrightarrow{\mathbf{y}} \in X^{\ell} \\
(\overrightarrow{\mathbf{x}}, \overrightarrow{\mathbf{y}})_{i}=\mathbf{p}}} e^{m \tau(\operatorname{supp}(\overrightarrow{\mathbf{x}}, \overrightarrow{\mathbf{y}}))} \kappa^{k} \lambda^{\ell}\left(\begin{array}{c}
k+\ell \\
\ell
\end{array}\right)|a(\overrightarrow{\mathbf{x}} \circ \overrightarrow{\mathbf{y}})| \\
& =\sum_{k, \ell \geq 0}\left(\begin{array}{c}
k+\ell \\
\ell
\end{array}\right) \kappa^{k} \lambda^{\ell} \max _{\mathbf{p} \in X} \max _{1 \leq i \leq k+\ell} \sum_{\substack{\overrightarrow{\mathbf{x}} \in X^{k}, \overrightarrow{\mathbf{y}} \in X^{\ell} \\
(\overrightarrow{\mathbf{x}}, \vec{y}) i}} e^{\mathrm{m} \tau(\operatorname{supp}(\overrightarrow{\mathbf{x}}, \overrightarrow{\mathbf{y}}))}|a(\overrightarrow{\mathbf{x}} \circ \overrightarrow{\mathbf{y}})| \\
& =\sum_{k, \ell \geq 0}\left(\begin{array}{c}
k+\ell \\
\ell
\end{array}\right) \kappa^{k} \lambda^{\ell} \max _{\mathbf{p} \in X} \max _{1 \leq i \leq k+\ell} \sum_{\substack{\mathbf{u} \in X^{k+\ell} \\
\overrightarrow{\mathbf{u}}_{i}=\mathbf{p}}} e^{\mathrm{m} \tau(\operatorname{supp}(\overrightarrow{\mathbf{u}}))}|a(\overrightarrow{\mathbf{u}})| \\
& =\sum_{n \geq 0}(\kappa+\lambda)^{n} \max _{\mathbf{p} \in X} \max _{1 \leq i \leq n} \sum_{\substack{\overrightarrow{\mathbf{u}} \in X^{n} \\
\overrightarrow{\mathbf{u}}_{i}=\mathbf{p}}} e^{\mathrm{m} \tau(\operatorname{supp}(\overrightarrow{\mathbf{u}}))}|a(\overrightarrow{\mathbf{u}})| \\
& =\|g\|_{\kappa+\lambda}
\end{aligned}
$$

\subsubsection{Other Related High Temperature Expansions}

The expansion treated in the main body of this section is just one of many similar expansions that are widely used in the construction and analysis of quantum field theories and many-body theories. Here are a few classes of such expansions. Don't take too seriously the names that I have assigned them - they do not have universally accepted meanings.

- Cluster expansions are expansions for unnormalized Schwinger functions, like for example $\int \varphi\left(z_{1}\right) \cdots \varphi\left(z_{n}\right) e^{-\mathcal{V}} d \mu(\varphi)$, that are used for proving the convergence of the infinite volume limit and of decay properties of the normalized Schwinger functions. See (Abdesselam and Rivasseau, 1995; Brydges, 1986; Glimm et al., 1973; Glimm and Jaffe, 1987; Rivasseau, 1991). 
- Mayer expansions are used to implement cancellations between the numerator and denominator in expressions like

$$
\frac{\int \varphi\left(z_{1}\right) \cdots \varphi\left(z_{n}\right) e^{-\mathcal{V}} d \mu(\varphi)}{\int e^{-\mathcal{V}} d \mu(\varphi)}
$$

(in the limit as the volume tends to infinity, such numerators and denominators tend to behave like the exponential of a constant times the volume) and are used for proving the convergence of the infinite volume limit and of decay properties of the normalized Schwinger functions. See (Abdesselam and Rivasseau, 1995; Brydges, 1986; Glimm et al., 1973; Glimm and Jaffe, 1987; Rivasseau, 1991).

- Polymer expansions. In its simplest form, a polymer expansion looks like

$$
1+\sum_{n=1}^{\infty} \frac{1}{n !} \sum_{\substack{X_{1}, \cdots X_{n} \subset x \\ x_{j} \neq \emptyset \text { for all } 1 \leq j \leq n \\ X_{i} \cap X_{j}=\emptyset \text { for all } i \neq j}} \prod_{\ell=1}^{n} A\left(X_{\ell}\right)
$$

Here each nonempty subset $X_{i}$ of the world $X$ is called a polymer and the function $A\left(X_{i}\right)$ is called a polymer activity. Two polymers $X_{i}$ and $X_{j}$ are said to be compatible if they are disjoint. Our expansion (1.52) was of this form. See (Brydges, 1986; Cammarota, 1982; Pordt, 1998; Kotecký and Preiss, 1986; Salmhofer, 1999; Simon, 1993) for lots of others. 


\section{Appendix A Complex Gaussian Integrals}

Integrals of polynomials times exponentials of quadratic functions are called Gaussian integrals and can be evaluated exactly. Lemma A.1, below, does so in our setting, where the integration variables are complex. To have integrals that actually exist, we consider an arbitrary, but finite, number, $L$, of complex integation variables. To compactify notation, we write

$$
\vec{\alpha}=\left(\alpha_{1}, \alpha_{2}, \cdots, \alpha_{L}\right) \quad\langle\vec{\beta}, \vec{\alpha}\rangle=\sum_{\ell=1}^{L} \beta_{\ell} \alpha_{\ell}
$$

Note that $\langle\vec{\beta}, \vec{\alpha}\rangle$ is not the usual complex inner product. We deliberately do not include a complex conjugate on the right hand side, so that all complex conjugates in our formulae appear explicitly.

To further compactify notation, we evaluate a generating functional. By repeatedly

differentating the conclusion of the lemma with respect to components of $\vec{\jmath}_{*}$ and $\vec{\jmath}$ and then setting $\vec{\jmath}_{*}=\vec{\jmath}=0$, you can create any polynomial you like downstairs on the left hand side. To be precise, suppose that $\mathcal{A}\left(\alpha^{*}, \alpha\right)$ is some action and set

$$
\mathcal{S}\left(\vec{\jmath}_{*}, \vec{\jmath}, \vec{\alpha}^{*}, \vec{\alpha}\right)=\left\langle\vec{\jmath}_{*}, \alpha\right\rangle+\left\langle\vec{\alpha}^{*}, \vec{\jmath}\right\rangle
$$

Define the expectation of $f\left(\vec{\alpha}^{*}, \vec{\alpha}\right)$ to be

$$
\left\langle f\left(\vec{\alpha}^{*}, \vec{\alpha}\right)\right\rangle=\frac{\int \prod_{\ell=1}^{L} \frac{d \alpha_{\ell}^{*} \wedge d \alpha_{\ell}}{2 \pi i} e^{\mathcal{A}\left(\alpha^{*}, \alpha\right)} f\left(\vec{\alpha}^{*}, \vec{\alpha}\right)}{\int \prod_{\ell=1}^{L} \frac{d \alpha_{\ell} * \wedge d \alpha_{\ell}}{2 \pi i} e^{\mathcal{A}\left(\alpha^{*}, \alpha\right)}}
$$

Then, for each $1 \leq m \leq L$,

$$
\left\langle\alpha_{m}\right\rangle=\left.\frac{\partial}{\partial j_{* m}}\left\langle e^{\mathcal{S}\left(\vec{\jmath}_{*}, \vec{\jmath}, \alpha^{*}, \alpha\right)}\right\rangle\right|_{\vec{\jmath}_{*}=\vec{\jmath}=0} \quad\left\langle\alpha_{m}^{*}\right\rangle=\left.\frac{\partial}{\partial j_{m}}\left\langle e^{\mathcal{S}\left(\vec{\jmath}_{*}, \vec{\jmath}, \alpha^{*}, \alpha\right)}\right\rangle\right|_{\vec{\jmath}_{*}=\vec{\jmath}=0}
$$

and, for each $1 \leq m, n \leq L$,

$$
\left\langle\alpha_{m}^{*} \alpha_{n}\right\rangle=\left.\frac{\partial^{2}}{\partial j_{m} \partial j_{* n}}\left\langle e^{\mathcal{S}\left(\vec{\jmath}_{*}, \vec{\jmath}, \alpha^{*}, \alpha\right)}\right\rangle\right|_{\vec{\jmath}_{*}=\vec{\jmath}=0}
$$




\section{Complex Gaussian Integrals}

Lemma A.1 Let $L \in \mathbb{N}$.

(a) Let $D$ be an $L \times L$ matrix whose real part, $D+D^{*}$, is strictly positive. That is, all of the eigenvalues of $\left[D_{\ell, \ell^{\prime}}+\overline{D_{\ell^{\prime}, \ell}}\right]_{1 \leq \ell, \ell^{\prime} \leq L}$ are strictly positive. Let $\vec{\jmath}_{*}, \vec{\jmath} \in \mathbb{C}^{L}$ and set

$$
\mathcal{A}\left(\alpha^{*}, \alpha\right)=-\left\langle\vec{\alpha}^{*}, D \vec{\alpha}\right\rangle \quad \text { and } \quad \mathcal{J}\left(\vec{\jmath}_{*}, \vec{\jmath}\right)=\left\langle e^{\mathcal{S}\left(\vec{\jmath}_{*}, \vec{\jmath}, \alpha^{*}, \alpha\right)}\right\rangle
$$

Then

$$
\mathcal{J}\left(\vec{\jmath}_{*}, \vec{\jmath}\right)=e^{\mathcal{C}\left(\vec{\jmath}_{*}, \vec{\jmath}\right)} \quad \text { where } \quad \mathcal{C}\left(\vec{\jmath}_{*}, \vec{\jmath}\right)=\left\langle\vec{\jmath}_{*}, D^{-1} \vec{\jmath}\right\rangle
$$

(b) Let $D, V$ and $W$ be $L \times L$ matrices with $V$ and $W$ self-transpose. That is, $V_{\ell, \ell^{\prime}}=$ $V_{\ell^{\prime} . \ell}$ and $W_{\ell, \ell^{\prime}}=W_{\ell^{\prime} . \ell}$. Assume that the matrix

$$
\left[\begin{array}{cc}
\frac{1}{2}\left(D+D^{*}\right) & V+\bar{W} \\
W+\bar{V} & \frac{1}{2}\left(D+D^{*}\right)^{t}
\end{array}\right]
$$

is strictly positive. Let $\vec{\jmath}_{*}, \vec{\jmath} \in \mathbb{C}^{L}$ and set

$$
\mathcal{A}\left(\alpha^{*}, \alpha\right)=-\left\langle\vec{\alpha}^{*}, D \vec{\alpha}\right\rangle-\left\langle\vec{\alpha}^{*}, V \vec{\alpha}^{*}\right\rangle-\langle\vec{\alpha}, W \vec{\alpha}\rangle \quad \text { and } \quad \mathcal{J}\left(\vec{\jmath}_{*}, \vec{\jmath}\right)=\left\langle e^{\mathcal{S}\left(\vec{\jmath}_{*}, \vec{\jmath}, \alpha^{*}, \alpha\right)}\right\rangle
$$

Then

$$
\mathcal{J}\left(\vec{\jmath}_{*}, \vec{\jmath}\right)=e^{\mathcal{D}\left(\vec{\jmath}_{*}, \vec{\jmath}\right)}
$$

where

$$
\begin{gathered}
\mathcal{D}\left(\vec{\jmath}_{*}, \vec{\jmath}\right)=\left\langle\vec{\jmath}_{*},\left(D-4 V\left(D^{t}\right)^{-1} W\right)^{-1} \vec{\jmath}\right\rangle-\left\langle W D^{-1} \vec{\jmath},\left(D-4 V\left(D^{t}\right)^{-1} W\right)^{-1} \vec{\jmath}\right\rangle \\
-\left\langle\vec{\jmath}_{*},\left(D-4 V\left(D^{t}\right)^{-1} W\right)^{-1} V\left(D^{t}\right)^{-1} \vec{\jmath}_{*}\right\rangle
\end{gathered}
$$

In the special case that $V=W$ and $V$ commutes with $D, \mathcal{D}$ simplifies to

$$
\begin{gathered}
\mathcal{D}\left(\vec{\jmath}_{*}, \vec{\jmath}\right)=\left\langle D \vec{\jmath}_{*},\left(D D^{t}-4 V^{2}\right)^{-1} \vec{\jmath}\right\rangle-\left\langle V \vec{\jmath},\left(D D^{t}-4 V^{2}\right)^{-1} \vec{\jmath}\right\rangle \\
-\left\langle V \vec{\jmath}_{*},\left(D^{t} D-4 V^{2}\right)^{-1} \vec{\jmath}_{*}\right\rangle
\end{gathered}
$$

Proof (a) The positivity condition on $D+D^{*}$ ensures that $D$ is invertible. (Otherwise, there would be a nonzero vector $\vec{v}$ with $D \vec{v}=0$ and hence $\left\langle\vec{v}^{*},\left(D+D^{*}\right) \vec{v}\right\rangle=0$, which would contradict the strict positivity of $D+D^{*}$.) We start by completing the square of the exponent in the numerator.

$$
\begin{aligned}
\mathcal{A}\left(\alpha^{*}, \alpha\right)+\mathcal{S}\left(\vec{\jmath}_{*}, \vec{\jmath}, \alpha^{*}, \alpha\right) & =-\left\langle\vec{\alpha}^{*}, D \vec{\alpha}\right\rangle+\left\langle\vec{\jmath}_{*}, \alpha\right\rangle+\left\langle\vec{\alpha}^{*}, \vec{\jmath}\right\rangle \\
& =-\left\langle\left(\vec{\alpha}^{*}-\left(D^{t}\right)^{-1} \vec{\jmath}_{*}\right), D\left(\vec{\alpha}-D^{-1} \vec{\jmath}\right)\right\rangle+\left\langle\vec{\jmath}_{*}, D^{-1} \vec{\jmath}\right\rangle
\end{aligned}
$$

At this stage, we have that

$$
\mathcal{J}\left(\vec{\jmath}_{*}, \vec{\jmath}\right)=e^{\mathcal{C}\left(\vec{\jmath}_{*}, \vec{\jmath}\right)} \frac{\int \prod_{\ell=1}^{L} \frac{d \alpha_{\ell}^{*} \wedge d \alpha_{\ell}}{2 \pi i} e^{-<\left(\vec{\alpha}^{*}-\left(D^{t}\right)^{-1} \vec{\jmath}_{*}\right), D\left(\vec{\alpha}-D^{-1} \vec{\jmath}\right)>}}{\int \prod_{\ell=1}^{L} \frac{d \alpha_{\ell * \wedge d \alpha_{\ell}}^{2 \pi i}}{2 \pi i} e^{-<\vec{\alpha}^{*}, D \vec{\alpha}>}}
$$

So it remains only to prove that the ratio of two integrals is exactly one. If $\vec{\alpha}$ and $\vec{\alpha}^{*}$ were independent integration variables, the change of variables $\vec{\alpha} \rightarrow \vec{\alpha}+D^{-1} \vec{\jmath}$, 
$\vec{\alpha}^{*} \rightarrow \vec{\alpha}^{*}+\left(D^{t}\right)^{-1} \vec{\jmath}_{*}$ would convert the numerator into exactly the integral that is in the denominator and we would be done. Unfortunately, $\vec{\alpha}$ and $\vec{\alpha}^{*}$ are not independent and, usually, $\left(D^{t}\right)^{-1} \vec{\jmath}_{*}$ is not the complex conjugate of $D^{-1} \vec{\jmath}$. Fortunately, with a little trickery, we can legitimately make the desired change of variables. First introduce a new, independent, vector of complex variables $\vec{\alpha}_{*}$. There is, in general, no requirement that $\vec{\alpha}_{*}$ be the complex conjugate of $\vec{\alpha}$. Replace all $\vec{\alpha}^{*}$ 's in the integral of the numerator by $\vec{\alpha}_{*}$ and choose as the domain of integration

$$
D_{\alpha}=\left\{\left(\vec{\alpha}_{*}, \vec{\alpha}\right) \in \mathbb{C}^{2 L} \mid \vec{\alpha}=\vec{\alpha}_{*}^{*}\right\}
$$

This recovers the original integral. That is,

$$
\begin{aligned}
\int_{\mathbb{C}^{L}} \prod_{\ell=1}^{L} \frac{d \alpha_{\ell}^{*} \wedge d \alpha_{\ell}}{2 \pi i} & e^{-<\left(\vec{\alpha}^{*}-\left(D^{t}\right)^{-1} \vec{\jmath}_{*}\right), D\left(\vec{\alpha}-D^{-1} \vec{\jmath}\right)>} \\
& =\int_{D_{\alpha}} \prod_{\ell=1}^{L} \frac{d \alpha_{*} \wedge d \alpha_{\ell}}{2 \pi i} e^{-<\left(\vec{\alpha}_{*}-\left(D^{t}\right)^{-1} \vec{\jmath}_{*}\right), D\left(\vec{\alpha}-D^{-1} \vec{\jmath}\right)>}
\end{aligned}
$$

Now make the change of variables

$$
\vec{\alpha}_{*}=\vec{z}_{*}+\left(D^{t}\right)^{-1} \vec{\jmath}_{*} \quad \vec{\alpha}=\vec{z}+D^{-1} \vec{\jmath}
$$

This gives

$$
\int \prod_{\ell=1}^{L} \frac{d \alpha_{\ell}^{*} \wedge d \alpha_{\ell}}{2 \pi i} e^{-<\left(\vec{\alpha}^{*}-\left(D^{t}\right)^{-1} \vec{\jmath}_{*}\right), D\left(\vec{\alpha}-D^{-1} \vec{\jmath}\right)>}=\int \prod_{D_{1}}^{L} \frac{d z_{*} \ell \lambda d z_{\ell}}{2 \pi i} e^{-\left\langle\vec{z}_{*}, D \vec{z}>\right.}
$$

with the domain

$$
D_{1}=\left\{\left(\vec{z}_{*}, \vec{z}\right) \in \mathbb{C}^{2 L} \mid \vec{z}=\vec{z}_{*}^{*}+\vec{\rho}\right\} \quad \text { with } \vec{\rho}=\left(D^{*}\right)^{-1} \vec{\jmath}_{*}^{*}-D^{-1} \vec{\jmath}
$$

In the next paragraph, we will use Stokes' theorem to show that we may replace the domain $D_{1}$ with the domain

$$
D_{0}=\left\{\left(\vec{z}_{*}, \vec{z}\right) \in \mathbb{C}^{2 L} \mid \vec{z}=\vec{z}_{*}^{*}\right\}
$$

Once that is done, we will have shown, this time legitimately, that the integral of the numerator in (A.4) is the same as the integral of the denominator, completing the proof.

Here are the details of the application of Stokes' theorem. Let $R$ be a large cutoff radius and define, for each $0 \leq t \leq 1$,

$$
D_{t, R}=\left\{\left(\vec{z}_{*}, \vec{z}\right) \in \mathbb{C}^{2 L}\left|\vec{z}=\vec{z}_{*}^{*}+t \vec{\rho}, \max _{1 \leq \ell \leq L}\right| z_{\ell} \mid \leq R\right\}
$$

Think of $\mathcal{B}=\bigcup_{t=0}^{1} D_{t}$ as a solid "cylinder". The boundary of $\mathcal{B}$ is the union of the top $D_{1, R}$ (which approachs $D_{1}$ as $R \rightarrow \infty$ ) and the bottom $D_{0, R}$ (which approachs $D_{0}$ as $R \rightarrow \infty)$ and the side 


\section{Complex Gaussian Integrals}

$C_{R}=\bigcup_{\ell=1}^{L} C_{R, \ell}$ with $C_{R, \ell}=\bigcup_{0 \leq t \leq 1}\left\{\left(\vec{z}_{*}, \vec{z}\right) \in \mathbb{C}^{2 L}\left|\vec{z}=\vec{z}_{*}^{*}+t \vec{\rho}, \max _{1 \leq \ell^{\prime} \leq L}\right| z_{\ell^{\prime}}|\leq R,| z_{\ell} \mid=R\right\}$

By Stokes' theorem, for any $2 L$-form $\omega$,

$$
\int_{\mathcal{B}} d \omega=\int_{D_{1, R}} \omega-\int_{D_{0, R}} \omega+\int_{C_{R}} \omega
$$

if $D_{1, R}$ and $D_{0, R}$ are oriented in the usual way and $C_{R}$ is oriented suitably. In our case, the form $\omega=\bigwedge_{\ell=1}^{L} \frac{d z_{* \ell} \wedge d z_{\ell}}{2 \pi i} e^{-\left\langle\vec{z}_{*}, D \vec{z}>\right.}$ obeys $d \omega=0$ (i.e. is closed) because $e^{-\left\langle\vec{z}_{*}, D \vec{z}\right\rangle}$ is an analytic function of $\vec{z}_{*}$ and $\vec{z}$. Hence

$$
\int_{D_{1, R}} \omega=\int_{D_{0, R}} \omega-\int_{C_{R}} \omega
$$

So we just have to show that $\int_{C_{R}} \omega$ converges to zero as $R \rightarrow \infty$. We start by bounding the integrand, or rather the real part of the exponent of the integrand. At any point on the side, $C_{R}, R \leq|\vec{z}| \leq \sqrt{L} R$ and there is a $0 \leq t \leq 1$ such that $\vec{z}_{*}=\vec{z}^{*}-t \vec{\rho}^{*}$ so that

$$
\begin{aligned}
\operatorname{Re}\left\langle\vec{z}_{*}, D \vec{z}\right\rangle & =\operatorname{Re}\left\langle\vec{z}^{*}, D \vec{z}\right\rangle-t \operatorname{Re}\left\langle\vec{\rho}^{*}, D \vec{z}\right\rangle \\
& =\frac{1}{2}\left\langle\vec{z}^{*},\left(D+D^{*}\right) \vec{z}\right\rangle-t \operatorname{Re}\left\langle\vec{\rho}^{*}, D \vec{z}\right\rangle \\
& \geq \frac{1}{2} \lambda_{0} R^{2}-\sqrt{L}|\vec{\rho}|\|D\| R
\end{aligned}
$$

where $\lambda_{0}$ is the smallest eigenvalue of $D+D^{*}$, assumed strictly positive, and $\|D\|$ is the operator norm of the matrix $D$.

We next bound the volume of the domain of integration. It suffices to do so for $C_{R, 1}$. The other $C_{R, \ell}$ 's can be treated in the same way. On $C_{R, 1}$, we have $\left|z_{1}\right|=R$. We may parametrize $z_{1}=R e^{i \theta}$, with $\theta$ running over $[0,2 \pi]$. Then $z_{*, 1}=R e^{-i \theta}-t \rho_{1}^{*}$ and

$$
d z_{1}=i R e^{i \theta} d \theta \quad d z_{*, 1}=-i R e^{-i \theta} d \theta-\rho_{1}^{*} d t \quad \frac{d z_{*, 1} \wedge d z_{1}}{2 \pi i}=-\frac{\rho_{1}^{*} R}{2 \pi} e^{i \theta} d t \wedge d \theta
$$

For all the other $\ell$ 's, we may parametrize $z_{\ell}=x_{\ell}+i y_{\ell}$ with $\left(x_{\ell}, y_{\ell}\right)$ running over $x_{\ell}^{2}+y_{\ell}^{2} \leq R^{2}$. Then $z_{*, \ell}=x_{\ell}-i y_{\ell}-t \rho_{\ell}^{*}$ and

$$
d z_{\ell}=d x_{\ell}+i d y_{\ell} \quad d z_{*, \ell}=d x_{\ell}-i d y_{\ell}-\rho_{\ell}^{*} d t
$$

Since there is already a $d t$ in $\frac{d z_{*, 1} \wedge d z_{1}}{2 \pi i}$ and $d t \wedge d t=0$,

$$
\bigwedge_{\ell=1}^{L} \frac{d z_{* \ell} \wedge d z_{\ell}}{2 \pi i}=-\frac{\rho_{1}^{*} R}{2 \pi} e^{i \theta} d t \wedge d \theta \bigwedge_{\ell=2}^{L} \frac{d x_{\ell} \wedge d y_{\ell}}{\pi}
$$

and

$$
\begin{aligned}
\left|\int_{C_{R .1}} \omega\right| & \leq\left|\rho_{1}\right| R \int_{0}^{1} d t \int_{0}^{2 \pi} \frac{d \theta}{2 \pi} \iint_{x_{2}^{2}+y_{2}^{2} \leq R^{2}} \frac{d x_{2} d y_{2}}{2 \pi} \cdots \iint_{x_{L}^{2}+y_{L}^{2} \leq R^{2}} \frac{d x_{L} d y_{L}}{2 \pi} \sup e^{-\operatorname{Re}<\vec{z}_{*}, D \vec{z}>} \\
& \leq\left|\rho_{1}\right| R\left(\frac{1}{2} R^{2}\right)^{L-1} e^{-\frac{1}{2} \lambda_{0} R^{2}+\sqrt{L}|\vec{\rho}|\|D\| R}
\end{aligned}
$$

This easily converges to zero as $R \rightarrow \infty$. 
(b) Once again, the main step is completing the square for the exponent of the numerator. We start my multiplying out

$$
\begin{gathered}
-\left\langle\left(\vec{\alpha}^{*}-\vec{J}_{*}\right), D(\vec{\alpha}-\vec{J})\right\rangle-\left\langle\left(\vec{\alpha}^{*}-\vec{J}_{*}\right), V\left(\vec{\alpha}^{*}-\vec{J}_{*}\right)\right\rangle-\langle(\vec{\alpha}-\vec{J}), W(\vec{\alpha}-\vec{J})\rangle \\
=-\left\langle\vec{\alpha}^{*}, D \vec{\alpha}\right\rangle-\left\langle\vec{\alpha}^{*}, V \vec{\alpha}^{*}\right\rangle-\langle\vec{\alpha}, W \vec{\alpha}\rangle+\left\langle\vec{\alpha}^{*},\left(D \vec{J}+2 V \vec{J}_{*}\right)\right\rangle \\
+\left\langle\left(D^{t} \vec{J}_{*}+2 W \vec{J}\right), \vec{\alpha}\right\rangle-\left\langle\vec{J}_{*}, D \vec{J}\right\rangle-\left\langle\vec{J}_{*}, V \vec{J}_{*}\right\rangle-\langle\vec{J}, W \vec{J}\rangle
\end{gathered}
$$

The first three terms are exactly $\mathcal{A}\left(\vec{\alpha}^{*}, \vec{\alpha}\right)$. The next two terms will form $\mathcal{S}\left(\vec{\jmath}_{*}, \vec{\jmath}, \vec{\alpha}^{*}, \vec{\alpha}\right)$ exactly provided

$$
\begin{aligned}
D \vec{J}+2 V \vec{J}_{*} & =\vec{\jmath} \\
D^{t} \vec{J}_{*}+2 W \vec{J} & =\vec{\jmath}_{*}
\end{aligned}
$$

Solving this pair of linear equations gives

$$
\begin{aligned}
\vec{J}_{*} & =\left(D^{t}-4 W D^{-1} V\right)^{-1}\left(\vec{\jmath}_{*}-2 W D^{-1} \vec{\jmath}\right) \\
\vec{J} & =\left(D-4 V\left(D^{t}\right)^{-1} W\right)^{-1}\left(\vec{\jmath}-2 V\left(D^{t}\right)^{-1} \vec{\jmath}_{*}\right)
\end{aligned}
$$

Substituting this in,

$$
\begin{aligned}
& \left\langle\vec{J}_{*}, D \vec{J}\right\rangle+\left\langle\vec{J}_{*}, V \vec{J}_{*}\right\rangle+\langle\vec{J}, W \vec{J}\rangle=\frac{1}{2}\left\langle\vec{J}_{*}, \vec{\jmath}\right\rangle+\frac{1}{2}\left\langle\vec{\jmath}_{*}, \vec{J}\right\rangle \\
& =\frac{1}{2}\left\langle\left(\vec{\jmath}_{*}-2 W D^{-1} \vec{\jmath}\right),\left(D-4 V\left(D^{t}\right)^{-1} W\right)^{-1} \vec{\jmath}\right\rangle \\
& \quad+\frac{1}{2}\left\langle\vec{\jmath}_{*},\left(D-4 V\left(D^{t}\right)^{-1} W\right)^{-1}\left(\vec{\jmath}-2 V\left(D^{t}\right)^{-1} \vec{\jmath}_{*}\right)\right\rangle \\
& =\left\langle\vec{\jmath}_{*},\left(D-4 V\left(D^{t}\right)^{-1} W\right)^{-1} \vec{\jmath}\right\rangle-\left\langle W D^{-1} \vec{\jmath},\left(D-4 V\left(D^{t}\right)^{-1} W\right)^{-1} \vec{\jmath}\right\rangle \\
& \quad-\left\langle\vec{\jmath}_{*},\left(D-4 V\left(D^{t}\right)^{-1} W\right)^{-1} V\left(D^{t}\right)^{-1} \vec{\jmath}_{*}\right\rangle \\
& =\mathcal{D}\left(\vec{\jmath}_{*}, \vec{\jmath}\right)
\end{aligned}
$$

Thus

$$
\mathcal{A}\left(\vec{\alpha}^{*}, \vec{\alpha}\right)+\mathcal{S}\left(\vec{\jmath}_{*}, \vec{\jmath}, \vec{\alpha}^{*}, \vec{\alpha}\right)=\mathcal{A}\left(\vec{\alpha}^{*}-\vec{J}_{*}, \vec{\alpha}-\vec{J}\right)+\mathcal{D}\left(\vec{\jmath}_{*}, \vec{\jmath}\right)
$$

The rest of the proof is very much like that of part (a), using in place of $\operatorname{Re}\left\langle\vec{z}^{*}, D \vec{z}\right\rangle=$ $\frac{1}{2}\left\langle\vec{z}^{*},\left(D+D^{*}\right) \vec{z}\right\rangle$ in the bound (A.5),

$\operatorname{Re}\left\{\left\langle\vec{z}^{*}, D \vec{z}\right\rangle+\left\langle\vec{z}^{*}, V \vec{z}^{*}\right\rangle+\langle\vec{z}, W \vec{z}\rangle\right\}=\frac{1}{2}\left[\left(\vec{z}^{t}\right)^{*} \vec{z}^{t}\right]\left[\begin{array}{cc}\frac{1}{2}\left(D+D^{*}\right) & V+\bar{W} \\ W+\bar{V} & \frac{1}{2}\left(D+D^{*}\right)^{t}\end{array}\right]\left[\begin{array}{c}\vec{z} \\ \vec{z}^{*}\end{array}\right]$ 


\section{References}

Abdesselam, A. and Rivasseau, V. (1995). Trees, forests and jungles: a botanical garden for cluster expansions. In Constructive Physics (Palaiseau, 1994), Lecture Notes in Physics, 446, pp. 7-36. Springer.

Balaban, T. (1995a). A low temperature expansion and "spin wave picture" for classical $N$-vector models. In Constructive Physics (Palaiseau, 1994), Lecture Notes in Physics, 446, pp. 201-218. Springer.

Balaban, T. (1995b). A low temperature expansion for classical $N$-vector models. I. A renormalization group flow. Comm. Math. Phys., 167, 103-154.

Balaban, T. (1996a). Localization expansions. I. Functions of the "background" configurations. Comm. Math. Phys., 182, 33-82.

Balaban, T. (1996b). A low temperature expansion for classical $N$-vector models. II. Renormalization group equations. Comm. Math. Phys., 182, 675-721.

Balaban, T. (1996c). The variational problems for classical $N$-vector models. Comm. Math. Phys., 175, 607-642.

Balaban, T. (1998a). The large field renormalization operation for classical $N$-vector models. Comm. Math. Phys., 198, 493-534.

Balaban, T. (1998b). A low temperature expansion for classical $n$-vector models. III. A complete inductive description, fluctuation integrals. Comm. Math. Phys., 196, $485-521$.

Balaban, T. (1998c). Renormalization and localization expansions. II. Expectation values of the "fluctuation" measures. Comm. Math. Phys., 198, 1-45.

Balaban, T., Feldman, J., Knörrer, H., and Trubowitz, E. (2008a). A functional integral representation for many boson systems. I: The partition function. Annales Henri Poincaré, 9, 1229-1273.

Balaban, T., Feldman, J., Knörrer, H., and Trubowitz, E. (2008b). A functional integral representation for many boson systems. II: Correlation functions. Annales Henri Poincaré, 9, 1275-1307.

Balaban, T., Feldman, J., Knörrer, H., and Trubowitz, E. (2009). Power series representations for bosonic effective actions. Journal of Statistical Physics, 134, 839-857.

Balaban, T., Feldman, J., Knörrer, H., and Trubowitz, E. (2010a). Power series representations for complex bosonic effective actions. I. a small field renormalization group step. Journal of Mathematical Physics, 51, 053305.

Balaban, T., Feldman, J., Knörrer, H., and Trubowitz, E. (2010b). Power series representations for complex bosonic effective actions. II. a small field renormalization group flow. Journal of Mathematical Physics, 51, 053306.

Balaban, T., Feldman, J., Knörrer, H., and Trubowitz, E. (2010c). The temporal ultraviolet limit for complex bosonic many-body models. Annales Henri Poincaré, 11, $151-350$. 
Berezin, F. A. (1966). The method of second quantization. In Pure Appl. Phys., vol 24. Academic Press.

Brydges, D. C. (1986). A short course on cluster expansions. In Phénomènes critiques, systèmes aléatoires, théories de jauge (Les Houches, 1984), pp. 129-183. NorthHolland.

Brydges, D. C. and Federbush, P. (1976). The cluster expansion in statistical physics. Commun. Math. Phys., 49, 233-246.

Brydges, D. C. and Federbush, P. (1977). The cluster expansion for potentials with exponential fall-off. Commun. Math. Phys., 53, 19-30.

Cameron, R. H. (1960). A family of integrals serving to connect the Wiener and Feynman integrals. J. Math. and Phys., 39, 126-140.

Cameron, R. H. (1962/1963). The Ilstow and Feynman integrals. J. Analyse Math., 10, 287-361.

Cammarota, C. (1982). Decay of correlations for infinite range interactions in unbounded spin systems. Comm. Math. Phys., 85, 517-528.

Durrett, R. (2010). Probability: theory and examples. Cambridge University Press.

Feldman, J., Knörrer, H., and Trubowitz, E. (2002). Fermionic functional integrals and the renormalization group. In CRM Monograph Series of the American Mathematical Society, 16. AMS.

Feldman, J. and Osterwalder, K. (1976). The Wightman axioms and the mass gap for weakly coupled $\left(\varphi^{4}\right)_{3}$ quantum field theories. Annals of Physics, 97, 80-135.

Fetter, A.L. and Walecka, J.D. (1971). Quantum Theory of Many-Particle Systems. McGraw-Hill.

Fröhlich, J. (1974). Verification of axioms for euclidean and relativistic fields and Haag's theorem in a class of $P(\phi)_{2}$ models. Annales Henri Poincaré, 21, 271-317.

Gel'fand, I. M. and Vilenkin, N.Ya. (1968). Generalized functions. Applications of harmonic analysis, Vol 4. Acad. Press.

Ginibre, J. (1965). Reduced density matrices of quantum gases. I. Limit of infinite volume. J. Math. Phys., 6, 238-251.

Ginibre, J. (1971). Some applications of functional integration in statistical mechanics. In Statistical Mechanics and Quantum Field Theory (Les Houches, 1970), pp. 327-427. Gordon and Breach.

Glimm, J. and Jaffe, A. (1987). Quantum physics: a functional integral point of view. Springer-Verlag.

Glimm, J., Jaffe, A., and Spencer, T. (1973). The particle structure of the weakly coupled $P(\phi)_{2}$ model and other applications of high temperature expansions, Part II: The cluster expansion. In Constructive Quantum Field Theory (Erice 1973), Lecture Notes in Physics, Vol 25, pp. 199-242. Springer.

Kotecký, R. and Preiss, D. (1986). Cluster expansion for abstract polymer models. Commun. Math. Phys., 103, 491.

Minlos, R. A. (1959). Generalized random processes and their extension to a measure. Trudy Moskov. Mat. Obšč, 8, 497-518.

Negele, J. W. and Orland, H. (1988). Quantum Many-Particle Systems. AddisonWesley.

Nelson, E. (1964). Feynman integrals and the Schrödinger equation. Journal of 


\section{References}

Mathematical Physics, 5, 332-343.

Pordt, A. (1998). Polymer expansions in particle physics. In Field Theoretical Tools for Polymer and Particle Physics (Lecture Notes in Physics, vol. 508), pp. 45-67. Springer.

Reed, M. and Simon, B. (1978). Methods of Modern Mathematical Physics, IV: Analysis of Operators. Academic Press.

Rivasseau, V. (1991). From Perturbative to Constructive Renormalization. Princeton University Press.

Rota, G.-C. (1964). On the foundations of combinatorial theory, I. Theory of Möbius functions. Z. Wahrscheinlichkeitstheorie Verw. Gebiete, 2, 340.

Salmhofer, M. (1999). Renormalization, an introduction. In Texts and Monographs in Physics. Springer.

Simon, B. (1993). The Statistical Mechanics of Lattice Gases, Volume 1. Princeton University Press.

Simon, B. (2005). Functional Integration and Quantum Physics. AMS Chelsea Publishing. 